\title{
An assessment of whether a carbon neutral initiative can successfully be implemented at
}

Victoria University of Wellington

\section{Tushara Kodikara}

Environmental Studies 593

A 90 point thesis submitted to the School of Geography, Environment, and Earth Studies, Victoria University of Wellington, as partial fulfilment of the degree of Masters of Environmental Studies

School of Geography, Environment and Earth Sciences

Victoria University of Wellington, 2007

March 2008 
The Hunter Building, Victoria University of Wellington, $13^{\text {th }}$ March 2008

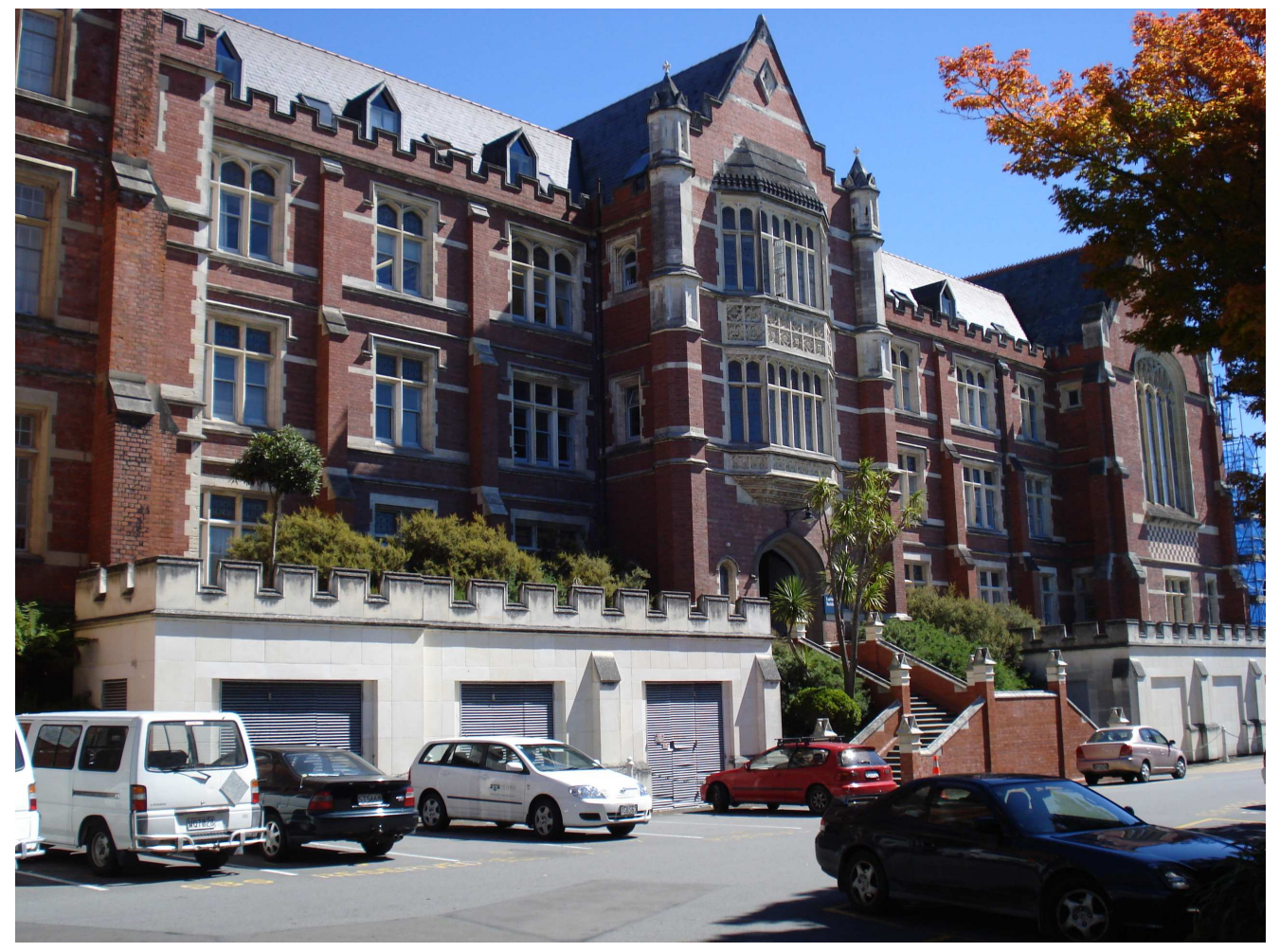

The world will not evolve past its current state of crisis by using the same thinking that created the situation

- Albert Einstein 


\begin{abstract}
The scientific evidence is now in no doubt - anthropogenic climate change has created a severe global problem and demands an urgent global response. The origin of anthropogenic climate change lies in the emission of greenhouse gases (GHG). Avoiding serious climate change will require reductions in GHG emissions from all sources. Universities can demonstrate leadership in this area by not only conducting research and teaching about climate change, but also by going further and starting to manage and mitigate their own impact on the climate.
\end{abstract}

This thesis assesses whether Victoria University of Wellington (VUW) can become carbon neutral and therefore have no net impact on climate change. Applying a corporate social responsibility model, the interface between the universities as teaching and research institutes and agents of change is investigated. This way, the opportunities, benefits and barriers in place for the university to become carbon neutral are identified and a framework to implement this initiative is developed.

The research found several potential short-term and enlightened self-interest benefits available to VUW in becoming carbon neutral. Despite this, many barriers will need to be overcome to achieve neutrality. The main obstacle is gaining a firm commitment from the University Council and senior management. If this commitment is achieved, then VUW is in a strong position to demonstrate leadership both at the level of the local Wellington community and nationally.

\title{
Key Words:
}

Victoria University of Wellington, carbon neutrality, university leadership, universities as agents of change, corporate social reasonability, university sustainability. 


\section{Acknowledgements}

I would like to thank my supervisor Ralph Chapman, who provided excellent support and advice during the development of this thesis. Special gratitude goes to all interviewees, who provided invaluable advice towards answering the aim of this thesis. Thanks must also go to Ian Shearer, Peter Barrett, VUWSA, Gecko, Daniel Cape, Derek Burrows, Rata Gordon and my fellow Masters of Environmental Studies colleagues, in particular, Nick Preval, Grace Leung, Amelie Goldberg, Sara Blank, Kata Duaibe, Hayley Vujcich and Patrick John. Most of all, I give thanks to my family and my partner Rachel. 


\section{Contents}

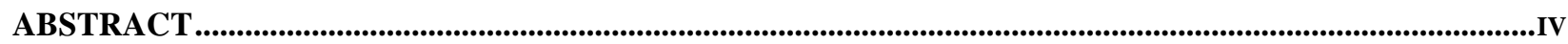

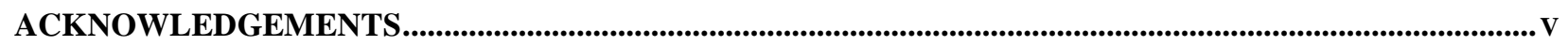

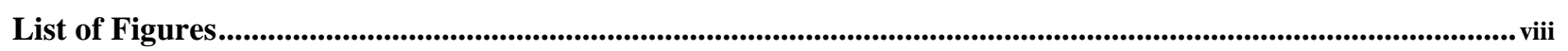

List of Tables ..................................................................................................................................................................... vii

List of Equations ........................................................................................................................................................ viii

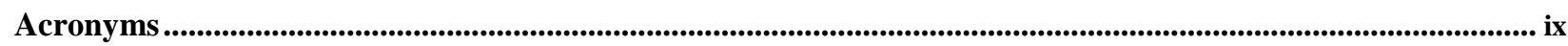

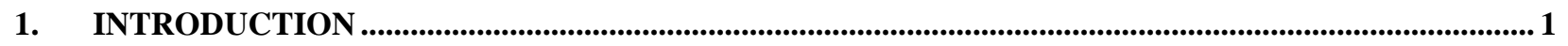

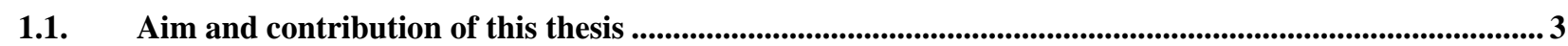

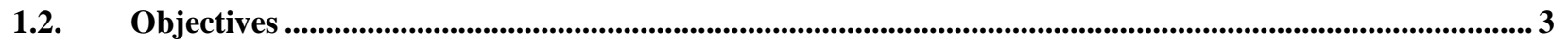

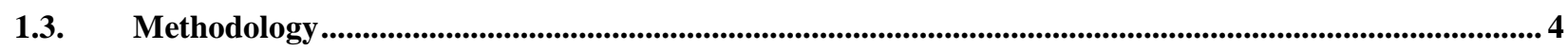

1.4. Conceptual Framework................................................................................................................................. 4

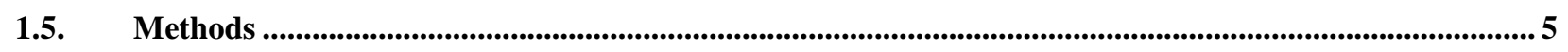

1.6. Other methods that could have been used to evaluate results .......................................................................... 7

1.7. Other carbon emissions management initiatives................................................................................ 7

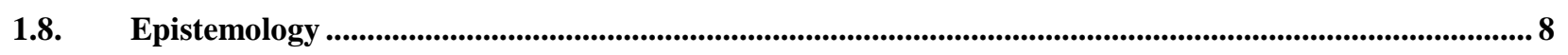

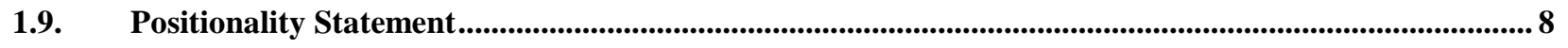

2. CARBON NEUTRALITY AND THE REASONS FOR IT ................................................................

2.1. The argument for reducing GHG emissions................................................................................................... 9

2.2. New Zealand's Emission Reduction Response ............................................................................. 16

2.3. Carbon Neutrality .......................................................................................................................................... 19

2.4. GHG Sinks and Sources ........................................................................................................................................ 20

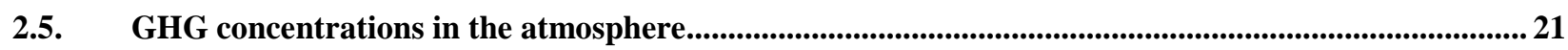

2.6. Rationale for carbon neutrality at VUW …….............................................................................................. 22

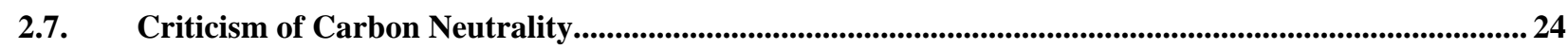

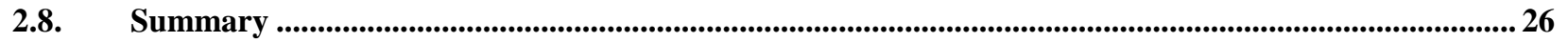

3. THE UNIVERSITY AS AN AGENT OF CHANGE AND THE CSR MODEL ........................................... 27

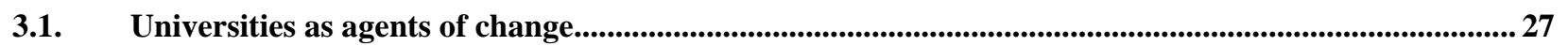


3.2. Corporate Social Responsibility and its application at Universities....................................................... 28

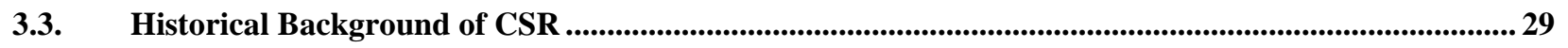

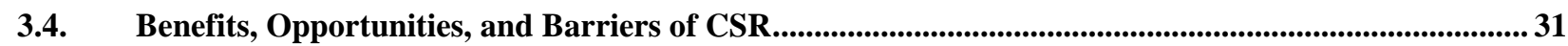

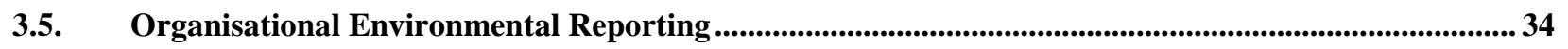

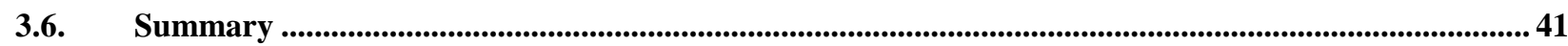

4. GHG EMISSIONS REDUCTION INITIATIVES AT UNIVERSITIES INTERNATIONALLY ........... 43

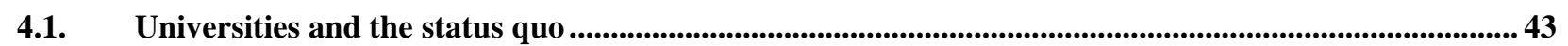

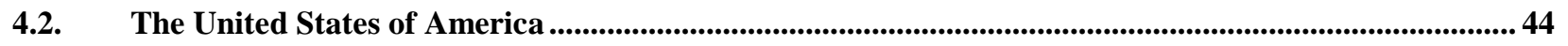

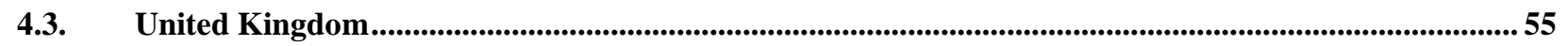

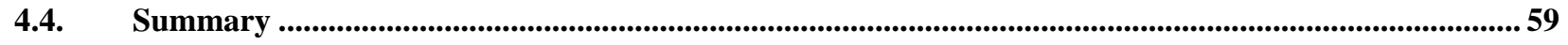

5. THE CARBON FOOTPRINT FOR VUW - CARBON AUDITING ....................................................61

5.1. Results: Identifying a 2006 GHG footprint for VUW ..........................................................................61

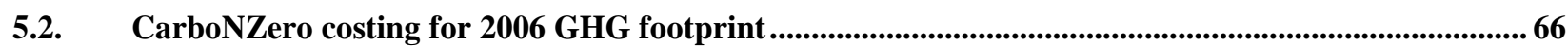

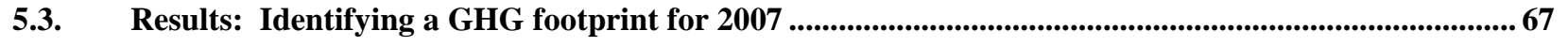

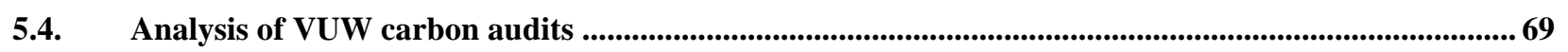

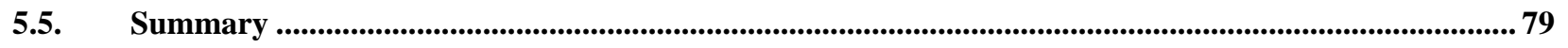

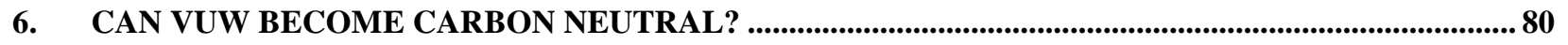

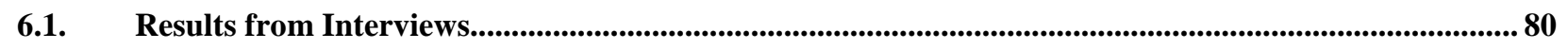

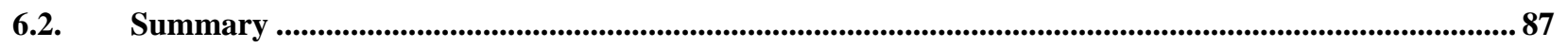

7. A FRAMEWORK FOR IMPLEMENTING A CARBON NEUTRAL INITIATIVE AT VUW ............. 88

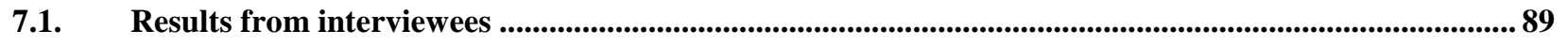

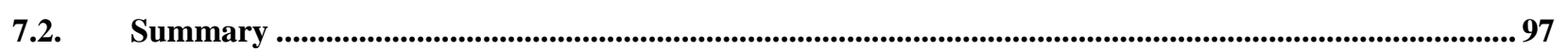

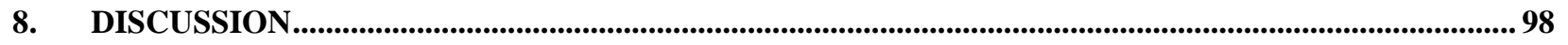

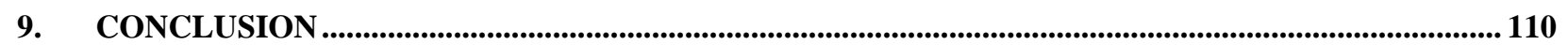

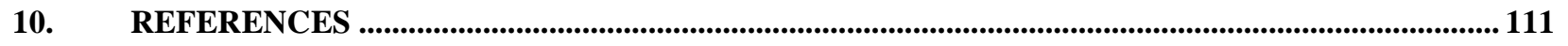

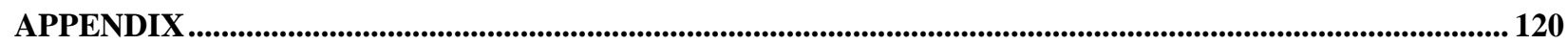




\section{List of Figures}

Figure 1: The interface between universities as research and teaching institutions, universities as agents as change and corporate social responsibility.....

Figure 2: $\quad$ Schematic of the Voluntary Retail Market (Taiyab, 2006, p.8) ..................................................... 14

Figure 3: Timeline of Universities and Colleges signed to ACUPCC (Begley, 2007) ..........................................45

Figure 4: 2008 College Sustainability Report Card Rating for the University of California, Santa Barbara ............ 49

Figure 5: 2008 College Sustainability Report Card Rating for Oberlin College ...............................................51

Figure 6: 2008 College Sustainability Report Card Rating for Harvard University- Cambridge, Massachusetts..... 53

Figure 7: 2008 College Sustainability Report Card Rating for Middlebury College ............................................5 54

Figure 8: The Higher Education Carbon Management Programme Five Step Process........................................... 57

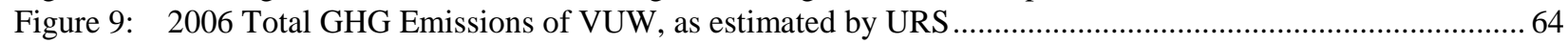

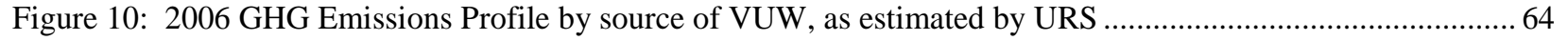

Figure 11: 2006 Total GHG Emissions of VUW, as estimated by NZBCSD assessment tool ................................ 66

Figure 12: 2006 GHG Emissions of VUW by source as estimated by NZBCSD assessment tool .......................... 66

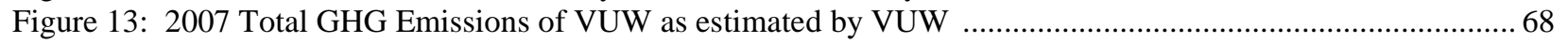

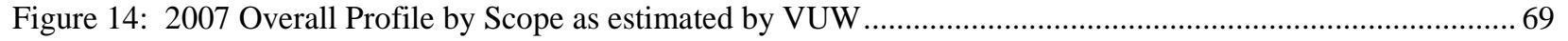

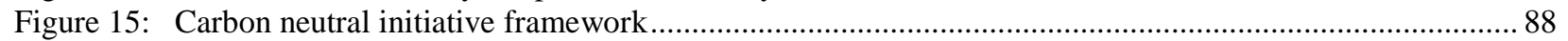

Figure 16: 2006 GHG Emissions Profile (excl. electricity) by source of VUW, as estimated by URS .................. 73

Figure 17: 2006 GHG Energy Emissions Profile of VUW, as estimated by URS ............................................ 74

\section{List of Tables}

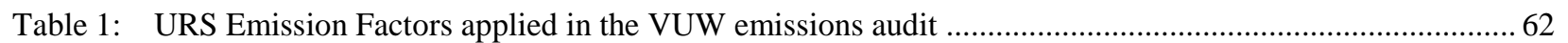

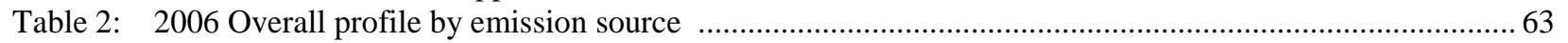

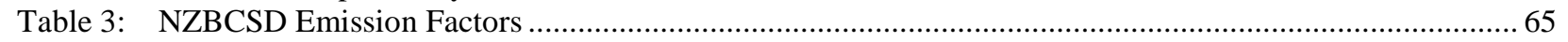

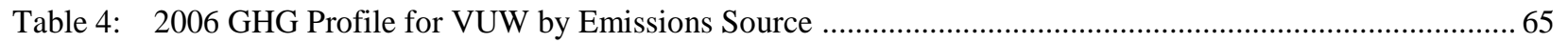

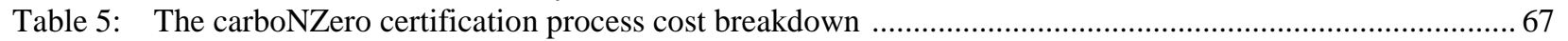

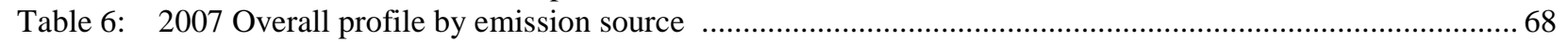

Table 7: Comparison between commuting emission factors between URS and NZBCSD ............................... 70

Table 8: Emission sources absent from the NZBCSD web based assessment tool ............................................71

Table 9: Adjusted VUW GHG emissions profile for NZBCSD estimate ......................................................... 72

Table 11: 2006 GHG Travel Emissions Profile (excl. electricity) of VUW, as estimated by URS ........................ 75

Table 12: Comparison of 2006/2007 VUW GHG Footprint (including electricity) ............................................78

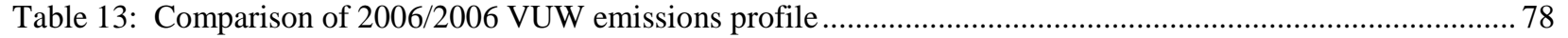

\section{List of Equations}

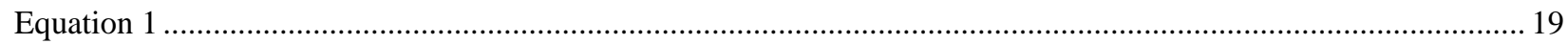

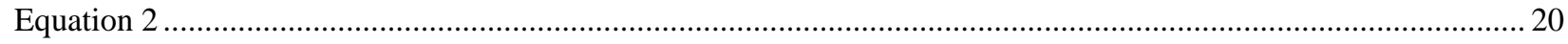

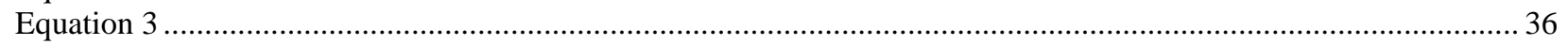




\section{Acronyms}

\begin{tabular}{|c|c|}
\hline AAUs & Assigned Amount Units \\
\hline ACUPCC & American College and University Presidents Climate Commitment \\
\hline 'BAU' & Business as Usual \\
\hline CCAT & Clean Air-Cool Planet Campus Climate Action Toolkit \\
\hline CCP-NZ & Communities for Climate Protection - New Zealand \\
\hline CDM & Clean Development Mechanism \\
\hline CERs & Certified Emission Reduction units \\
\hline $\mathrm{CO}_{2}$ & Carbon Dioxide \\
\hline $\mathrm{CO}_{2} \mathrm{e}$ & Carbon dioxide equivalent \\
\hline CSR & Corporate Social Responsibility \\
\hline CSRC & College Sustainability Report Card \\
\hline ERUs & Emission Reduction Units \\
\hline EPA & US Environmental Protection Agency \\
\hline ETS & Emissions Trading Scheme \\
\hline FAR & Fourth Assessment Report \\
\hline GHG & Greenhouse Gas \\
\hline HECM & Higher Education Carbon Management \\
\hline ICC & The International Chamber of Commerce \\
\hline IPCC & International Panel on Climate Change \\
\hline ISO & International Organisation for Standardisation \\
\hline $\mathrm{JI}$ & Joint Implementation \\
\hline MED & Ministry of Economic Development \\
\hline MfE & Ministry for the Environment \\
\hline NGOs & Non-governmental organisation \\
\hline NZBCSD & New Zealand Business Council for Sustainable Development \\
\hline TAR & Third Assessment Report \\
\hline TEC & Tertiary Education Commission \\
\hline $\mathrm{UCL}$ & University College London \\
\hline UCSB & University of California, Santa Barbara \\
\hline UK & United Kingdom \\
\hline UNCED & United Nations Conference on Environment and Development \\
\hline UNEP & United Nations Environment Programme \\
\hline UNFCCC & United Nations Framework Convention on Climate Change \\
\hline UPS & United Parcel Service \\
\hline URS & URS New Zealand Limited \\
\hline USA & United States of America \\
\hline VCS & Voluntary Carbon Standard \\
\hline VERs & Verified Emission Reductions \\
\hline VUW & Victoria University of Wellington \\
\hline VUWSA & Victoria University of Wellington Students' Association \\
\hline WBCSD & World Business Council on Sustainable Development \\
\hline WCC & Wellington City Council \\
\hline WRI & World Resources Institute \\
\hline
\end{tabular}




\section{Introduction}

Based on scientific evidence, the need to manage and mitigate greenhouse gas (GHG) emissions is clear. The global average air temperature near the Earth's surface has risen over the last century. If anthropogenic GHG emissions continue to increase, then it is projected that global temperature, sea levels and the intensity of extreme weather events will increase. There will be a change to the amount and pattern of precipitation. Other potential effects include changes in agricultural yields, glacier retreat, many species' extinctions and an increase in the ranges of disease vectors (IPCC, 2007a).

To prevent the potentially catastrophic effects of climate change, anthropogenic GHG emissions need to be significantly reduced (Bode, 2006). Climate scientist, James Hanson states that "if humanity wishes to preserve a planet similar to that on which civilization developed, paleoclimate evidence and ongoing climate change suggest that carbon dioxide $\left(\mathrm{CO}_{2}\right)$ will need to be reduced from its current 385 ppm to at most 350 ppm” (Hansen, et al, 2008: 1).

For this to happen, changes of an unparalleled scope and scale - institutional, technological and behavioural - need to occur. Rigorous action will be needed sooner rather than later in order to meet stringent targets, otherwise the environmental, economic and social costs of climate change are likely to be greater than those of any challenge yet to have faced humanity (Stern, et al, 2006).

In this regard, universities have an important role in society, at the forefront of not only climate science but in all areas of higher education and research. The traditions of western universities can be traced back to the practices of the Islamic madrasas. Later, the medieval emergence of the university incorporated the classical Greek and Roman ideas of self-governing citizenry (Howden-Chapman, 2006). Traditionally, universities are academically autonomous from the rest of society. However, universities are no longer placid backwaters serving established elites, but part of the mainstream of society. Institutions of higher education are being called upon to perform unparalleled new functions - develop new technologies; produce tomorrow's leaders and citizens; and become relevant to society's needs and wants (Albtach, 1972).

Universities have played a leadership role in addressing important societal issues, such as civil rights, free speech and thinking, foreign policy, and, in particular, the anti-war movement. This is 
a reflection of universities' role as centres for intellectual inquiry, critical thinking and innovation. As universities are not restricted in the way that other public and private organisations are, they have the freedom to play a leadership role regarding issues and thinking that question the status quo. This is true of leadership on climate change; not only in researching and teaching on climate change science and policy, but in taking practical steps to mitigate the effects of climate change (Ahmed, et al, 2006).

Victoria College was founded through an Act of Parliament in 1897. In 1962 Victoria College became Victoria University of Wellington (VUW). VUW now has a community of almost 25,000 and four campuses located throughout Wellington. In 2006, over 21,000 students were enrolled and 1906 full-time equivalent staff were employed (VUW, 2007). Like all tertiary institutes in New Zealand, VUW is primarily government funded, with student fees, donations and investments making up its remaining revenue.

In early 2008, the government introduced a new approach to funding the tertiary education system. Prior to this, the funding system was based on the number of students attending each institution. The new system promotes a stronger focus on the quality and relevance of education and research outcomes. Government expenditure is no longer demand driven, but instead based on a three-year funding path. This is justified by ensuring that tertiary education resources are focused on courses and research that meet the needs of New Zealand (TEC, 2007).

Bowing to pressure from students and staff to improve its environmental record, VUW has only recently developed an environmental policy and on the basis of this created an environmental committee to implement this policy (VUW, 2006a). The purpose of the policy is to:

\footnotetext{
"Actively apply the concepts of sustainability and environmental awareness of the local and global environment. This policy defines the environmental obligations of the University and considers how implementation of the policy should occur. The environmental policy is consistent with the University's Strategic Plan and Charter and will enhance Victoria's place as a leading Australasian research and teaching institution. Commitment to this environmental policy will be met by demonstrating leadership in applying environmentally responsible practices for the purpose of sustainability."
}

The overarching principles of the policy are to recognise the University's responsibility to manage its environmental impact, implement sustainable and environmentally sound business practices, ensure the University's footprint is managed, provide community leadership and environmental awareness and have a commitment to implementing the University's Treaty of 
Waitangi statute (VUW, 2006a). However, it has made no official commitment to reduce GHG emissions produced by its operations.

In November 2006, at the launch the book of Confronting Climate Change: Critical Issues for New Zealand, VUW's Chancellor, Emeritus Professor Tim Beaglehole gave a speech and asked the question "The University has recognised that in planning future buildings it must give consideration to issues of environmentally sustainable design. Should it go further and consider steps necessary to make itself carbon neutral?" (Speech, Beaglehole, 2006)

This thesis seeks to answer this question by explaining whether and how universities can provide both practical and ethical leadership in terms of climate change. Several universities around the world have already begun to considerably reduce their own emissions of GHGs by making a commitment to carbon neutrality. This thesis explores the proposition that VUW can, to its own advantage, go further than teaching about and researching climate change. It can start managing and mitigating its climate impact and achieve carbon neutrality.

\subsection{Aim and contribution of this thesis}

The aim of this thesis is to assess whether a carbon neutral initiative can successfully be implemented at Victoria University of Wellington.

This thesis builds on previous studies on decision-making and change processes at higher learning institutions in terms of environmental initiatives (Shriberg, 2002; Dautremont-Smith, 2003; Bardati, 2006; Bekessy, et al, 2007). The first contribution that this thesis makes is developing the theme that universities are agents of change within society. Secondly, it investigates the necessary drivers needed for change and the barriers that prevent change.

\subsection{Objectives}

The specific objectives of this thesis are to:

- Identify what has been done at universities internationally, in terms of GHG emission reduction initiatives (Chapter 4);

- Investigate a GHG footprint for Victoria University of Wellington, including methodological issues associated with this (Chapter 5); 
- Identify the perceived opportunities for and benefits of the university implementing a carbon neutral initiative and the barriers to this (Chapter 6);

- Identify and evaluate a framework for VUW to implement a carbon neutral initiative (Chapter 7).

\subsection{Methodology}

In fulfilling the aim and objectives, a wide body of literature was reviewed; information was gathered from a conference and from key stakeholders within the university and representatives of organisations that have already adopted the goal of carbon neutrality / carbon reduction targets were interviewed.

This thesis uses a mixed methods research format and follows a 'three-tier' approach (Creswell and Plano-Clark, 2007). In the first tier, case studies were examined to establish a baseline understanding of universities as agents of change. The second tier used a quantitative research method to establish a GHG footprint for VUW. The third tier used a qualitative research method, involving interview observations.

\subsection{Conceptual Framework}

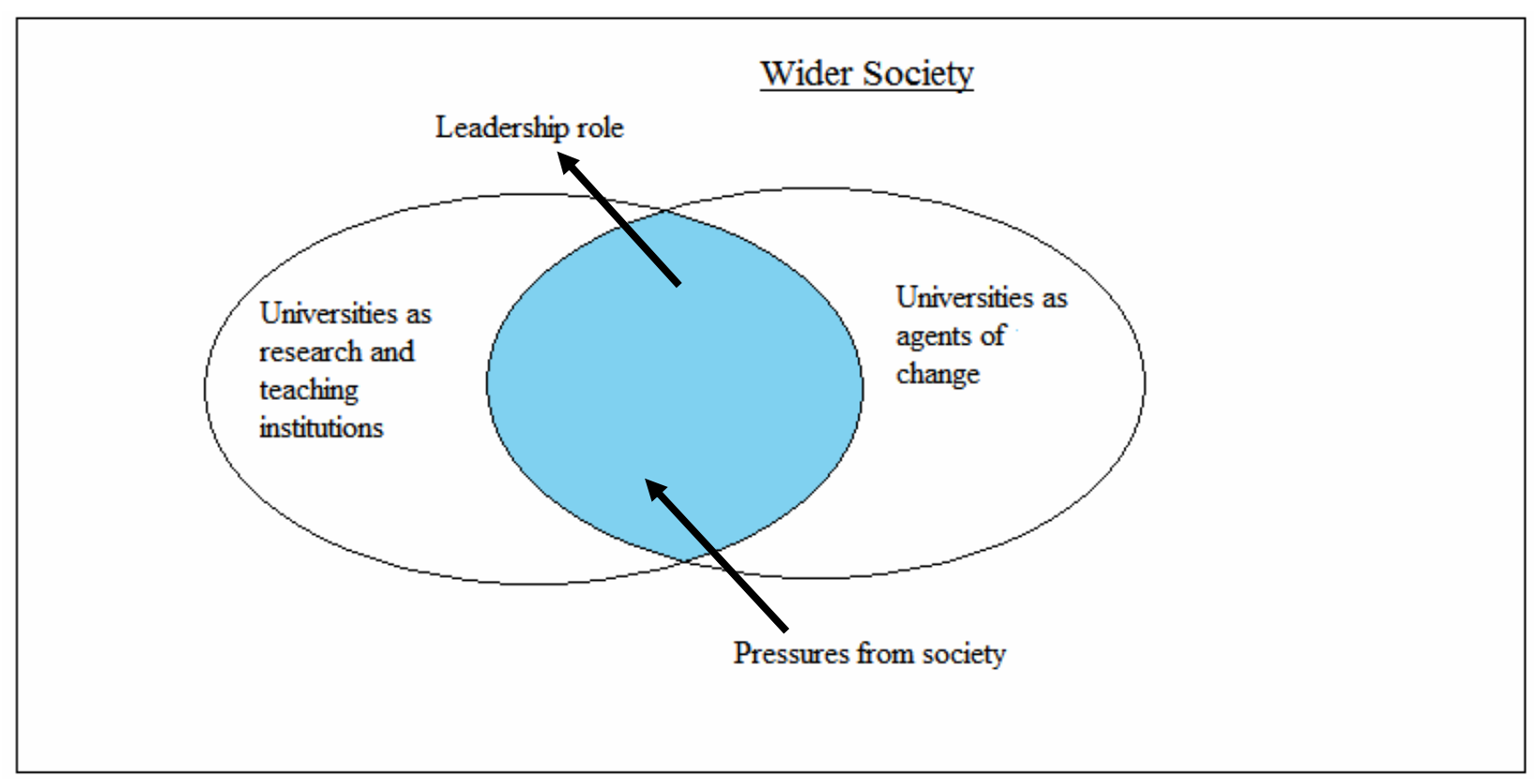

Figure 1: The interface between universities as research and teaching institutions, and universities as agents of change, using a corporate social responsibility model. 
The basis for the conceptual framework used in this thesis uses the idea of corporate social responsibility (CSR) as a business model to examine the leadership interface between the conventional role of universities and the role of universities as agents for change (Figure 1). This frames the investigation of the issues associated with implementing an environmental initiative such as carbon neutrality at VUW.

\subsection{Methods}

\section{Objective one}

Objective one involved investigating whether universities internationally have already committed to becoming carbon neutral and what this involves. A literature review and personal correspondences were used to meet this objective.

\section{Objective two}

Objective two involved examining VUW's GHG footprint by reviewing VUW's recent environmental audit by URS New Zealand Ltd (URS). The New Zealand Business Council for Sustainable Development's web-based carbon footprint assessment tool was used as a comparison.

\section{Objectives three and four}

To answer both objectives three and four, interviews were conducted amongst various stakeholders involved in decision-making roles at VUW and with other organisations that have made commitments to becoming carbon neutral. The purpose of the interviews was two-fold. Firstly the purpose was to ascertain the perceived opportunities for and the benefits of the university implementing a carbon neutral initiative and the barriers to this. Secondly, interviews were conducted to identify a framework for implementing a carbon neutral strategy at VUW. 


\subsubsection{Participants interviewed for this thesis}

Over the course of the 2007 calendar year, twelve key stakeholders involved in decision-making at VUW were interviewed on the issue of carbon neutrality at the university. VUW interviewees included Andrew Wilks, Environmental Manager Facilities Management and chair of the VUW Environmental Committee; Jenny Bentley, Director, Facilities Management; Rainsforth Dix, Manager, Student Union Complex; Professor Charles Daugherty, Assistant Vice-Chancellor (Research), University Council member; Professor David Bibby, Pro-Vice Chancellor, Dean of Science, Dean Architecture and Design; Kevin Duggan, Management Information Analyst, University Council member; Professor Jonathan Boston, Deputy Director of the Institute of Policy Studies; and Phillip Barker, the Co-leader of Gecko, the VUW student environmental club.

Interviewees involved with other organisations that have committed to a carbon neutral target include Melanie Hutton, former climate change campaigner, World Wildlife Foundation; Diana Shand, Communities for Climate Protection - New Zealand (CCP-NZ); Ian Shearer, (CCP-NZ); Caitlin Littlefield, Middlebury College, Vermont; and Roddy Yarr, St Andrews University, Scotland.

Interviews were conducted in a semi-formal manner and lasted no longer than 45 minutes. A semi-structured interview approach was chosen, because such interviews are more likely to evoke the interviewees' viewpoints than is the case with standardised interviews, which tend to be restrictive (Dunn, 2005).

A range of interview questions was asked based on the literature review described in this thesis (see Appendix). Qualitative data were analysed by coding and categorising the responses into major conceptual areas. An 'open coding' approach was used, as no pre-conceived categories existed before the interviews took place. Using the scissors and paste method, statements were grouped together by their content significance to create broad categories. For example, statements concerning the monetary constraints to implementing a carbon neutral initiative were grouped into 'Financial Barriers', while statements regarding ratios between students and staff were grouped into 'Academic priorities'. 
Ethics approval was given for the interview component of this thesis. All interviewees were provided with a copy of the information sheet and consent form. All interviewees gave their consent to be named and their opinions to be attributed (see Appendices).

\subsection{Other methods that could have been used to evaluate results}

For this research, there are very few alternative methods that could be used to address the aim of this thesis. Quantitative surveys could have been sent out to various members of the university community and the community at large to gather results; however due to time restriction, this was not practical.

If the primary aim of this thesis had been to investigate the economic feasibility of a carbon neutral initiative, then a cost-benefit analysis would have been an appropriate method to use. However, the aim is not limited to economic factors affecting decision-making at a university. As discussed in the literature review in the next two chapters, there is a range of factors, often largely qualitative in nature that can affect decision-making at a university.

\subsection{Other carbon emissions management initiatives}

There are other options VUW can choose to manage GHG emissions produced from its operations apart from aiming for carbon neutrality. Some of these options include having percentage emissions reductions each year, or setting emission targets. Percentage reductions would mean choosing a base year or working from the previous year, i.e. each year emissions could be reduced by 10 percent of emissions produced in the previous year. A base year approach would be akin to Kyoto targets. For example, by 2015 emissions could aim to be reduced by 20 percent compared to emissions produced in 2006.

However, both of these 'specified level' options would restrict the university in terms of growth, as the University would be pledging to constraining itself to a set amount of GHG emissions each year. Also, choosing either of these options would mean that the University would have a positive net GHG footprint each year, adding to the increasing GHG emissions in the atmosphere currently occurring. As explained in the next chapter, carbon neutrality is the only option that aims for a zero net GHG footprint. 


\subsection{Epistemology}

This research follows in the epistemological philosophy of environmental pragmatism, referring to finding those philosophies which work in practice to explain and solve environmental problems (Rothenberg, 1996). It is an open-ended and dynamic inquiry into specific real-life problems of humanity's relationship with the environment. It directs a decision-maker to choose a political strategy which advantageously results in solutions to environmental problems (Light and Katz, 1996).

When it comes to policy design, the pragmatic view is to continue experimenting with ways to reconstruct social institutions so that the public has a say in determining the kind of environment we inhabit (Parker, 1996). Pragmatism views individuals as the source of genuine insight into what is needed and accordingly tries to maximise participation in governing (Parker, 1996).

\subsection{Positionality Statement}

I am in favor of Victoria University becoming carbon neutral. It should be acknowledged that I bring a certain bias and subjectivity to issues discussed in this thesis, but I have done my utmost to keep my personal views independent of the process of data collection and analysis.

I was the 2006/ 2007 Victoria University of Wellington Students' Association Environmental Officer. I sat on both the VUW Environmental Committee ${ }^{1}$ and Transport Committee and I am an active member of Gecko, the university's student environmental club. I had already met and developed relationships with several of the people I interviewed for this thesis; nevertheless, I do not believe that this has significantly biased the results or conclusions I have drawn.

\footnotetext{
${ }^{1}$ Other members of the 2007 Environmental Committee included Andrew Wilks, VUW Environmental Manager; Associate Professor Ralph Chapman, Director of Environmental Studies, Kelburn campus representative; Dr. Christian Schott, Senior Lecturer, Pipitea Campus representative; Associate Professor John Storey, Deputy Dean of the Faculty of Architecture and Design, Te Aro campus representative; Vasilisa Frolova, Finance representative; Dave Povey, Faculty of Management representative; Jennie Calder-Smith, Faculty Manager, Education, Karori campus representative; Aaron Packard, Gecko / Student representative.
} 


\section{Carbon neutrality and the reasons for it}

This chapter analyses the issues surrounding the potential for Victoria University to become carbon neutral. The chapter begins by outlining the scientific and the political agenda, in both an international and domestic context. Following this, the concept of carbon neutrality is defined and the rationale for carbon neutrality is discussed.

\subsection{The argument for reducing GHG emissions}

\subsubsection{The problem of climate change under a timeline / scenario of 'Business as Usual'}

Scientists have been aware of anthropogenic climate change for over a century (Tyndall, 1861; Arrhenius, 1896; Callendar, 1938; Revelle and Suess, 1957). Nevertheless, progress in mitigating these effects has been slow. While there is a worldwide scientific consensus that atmospheric GHG emissions have to decrease, worldwide GHG levels are still increasing. Global GHG emissions due to human activities have grown since pre-industrial times, with an increase of 70 percent occurring between 1970 and 2004 (IPCC, 2007a: 4).

In 1984, the International Conference on the Assessment of the Role of Carbon Dioxide and of other Greenhouse Gases in Climate Variations and Associated Impacts was held in Villach, Austria. There were 89 scientists from 23 countries in attendance and they declared a threat greater then any other in human history could occur in the first half of the $21^{\text {st }}$ century. They warned that this potential catastrophe would be overwhelmingly influenced by government policies. They called for consideration of a global convention to tackle the impending threat (Pearce, 2005).

However, anthropogenic climate change only began to enter the political arena in 1988 when NASA climate change scientist James Hansen published a paper in the Journal of Geophysical Research (Hansen, et al, 1988) and submitted a report to the US Congress warning that climate change would become a problem in the near future. Twenty years on, his predictions of rising temperatures due to human activity have proved to be correct (Renowden, 2007).

Also in 1988, the United Nations Environmental Programme (UNEP) and the World Meteorological Organisation established the Intergovernmental Panel on Climate Change (IPCC) 
to provide independent scientific advice on the complex and important issue of climate change from a scientific, socio-economic and policy perspective.

The IPCC has published four comprehensive assessments and several special reports scrutinising scientific climate change research, the predicted impacts and opportunities for adaptation and mitigation. The reports have all concluded that anthropogenic climate change is a real phenomenon, each subsequent report stating this with a growing level of certainty. These reports have served as the basis for negotiating the United Nations Framework Convention on Climate Change (UNFCCC) (IPCC, 2007a).

The UNFCCC (1992) sets out a broad framework for intergovernmental efforts to tackle climate change. 191 countries have ratified the Convention. It recognises that climate change is a global issue and that GHG emissions need to be cut by all parties. The Convention came into force on 21 March 1994.

The scientific understanding of climate change has continued to improve vastly since 1988 . The release of the Third (TAR 2001) and Fourth (FAR 2007) Assessment Reports of the IPCC. Working Group 1 (The Physical Science Basis) of the FAR built on the findings of the TAR with large amounts of new and more comprehensive data, more sophisticated analysis of data, improvements in understanding of processes and their simulation in models and a more extensive exploration of uncertainty ranges (IPCC, 2007a: 10).

The report concluded that:

"Most of the observed increase in global average temperatures since the mid- $20^{\text {th }}$ century is very likely due to the observed increase in anthropogenic greenhouse gas concentrations. This is an advance since the TAR's conclusion that "most of the observed warming over the last 50 years is likely to have been due to the increase in greenhouse gas concentrations."

Anthropogenic climate change is a problem of the public good as it involves a key part of the 'global commons' - the atmosphere (Manne and Stephan, 2005). It may be the most important problem that human civilisation faces this century. Regrettably though, that which drives climate change - GHG emissions from energy use and land use changes - are vital components of the current global economic system (Perman, 1994; Ahmed, 2006). By way of GHG emissions, human activity is influencing other aspects of the climate, including oceanic temperature, 
continental-average and extreme temperatures, and wind patterns. It is the pervasive nature of this series of effects that makes addressing the consequences of climate change particularly critical (IPCC, 2007c).

According to the Stern Review, edited by Sir Nicholas Stern, taking strong action to reduce emissions has to be viewed as an investment, a cost incurred now and in the next few decades to prevent the risks of very severe consequences in the future (Stern, et al, 2006). If these investments are made intelligently, the costs will be manageable and there will be a wide range of opportunities for sustained growth and development in due course. Applying this principle at the level of the university is the essential objective of this research.

The global solution to the climate change problem will require a variety of collectively agreed international initiatives. Actions by some will not be adequate. The Earth's atmosphere is a public good; therefore the issue of free riding occurs. Without clear and enforceable property rights, every nation exploits the atmosphere, using it as a dumping ground for GHGs. Likewise, if emitters refuse to cooperate, or take action, the action of others involved will be less effective. Thus, a high level of cooperation, including meaningful participation by all significant emitters, will be required to mitigate climate change.

Both mitigation and adaptation to climate change represent adjustments to changing climatic (environmental) conditions (Stechr and von Storch, 2005). However, they represent two fundamentally dissimilar approaches. The differences and potential conflict between the two approaches are now well documented (Klein, et al, 2005), and are an important aspect of the climate change literature (Cohen, et al, 1998).

On the one hand, mitigation can be described as protecting nature from society, reducing the risk of severe climate change. Adaptation, on the other hand, can be described as protecting society from nature, reducing the harm placed on society from climatic changes (Stechr and von Storch, 2005). In terms of the precautionary principle, there exists a general consensus that mitigation is the favoured of the two options; that emission reduction, as opposed to following 'business as usual' ('BAU') emissions, with adaptation to subsequent changes, is the most appropriate measure to deal with climate change. In order to avoid the serious consequences of climate change, industrialised countries need to reduce yearly emissions by 60 to 80 percent of what they currently are by 2050 (IPCC, 2007d: 172). 


\subsubsection{International Mechanisms and Markets Supporting Emission Reductions}

\section{The Kyoto Protocol}

There is a consensus that GHG emissions should be limited in order to prevent negative impacts from climate change (Bode, 2006). Article 2 of the United Nations Framework Convention on Climate Change (UNFCCC, 1992) specifies the objective as follows:

\footnotetext{
"The ultimate objective of this Convention and any related legal instruments that the Conference of the Parties may adopt is to achieve, in accordance with the relevant provisions of the Convention, stabilisation of greenhouse gas concentration in the atmosphere at a level that would prevent dangerous anthropogenic interference with the climate system. Such a level should be achieved within a time-frame sufficient to allow ecosystems to adapt naturally to climate change, to ensure that food production is not threatened and to enable economic development to proceed in a sustainable manner."
}

The 1997 Kyoto Protocol builds upon the UNFCCC and shares the Convention's objective, principles and institutions. While not prescriptive, the Protocol allows for an international 'cap and trade' system, and sets out legally binding targets and timetables to reduce GHG emissions for countries that have ratified it. The first commitment period of the Kyoto Protocol began on $1^{\text {st }}$ of January 2008 and expires on the $31^{\text {st }}$ of December 2012 (UNFCCC, 1998).

Countries ratifying the Protocol fall into two groups, Annex I and Annex II. Annex I countries have legally binding quantified targets to reduce their emissions, whereas Annex II countries, developing nations, are encouraged but are not legally required to reduce emissions.

To date, 174 countries, the most recent being Australia in late 2007, have signed and ratified the Protocol; nevertheless, one of the world's largest emitters, the United States of America (USA), despite signing the Protocol, is yet to ratify it. Other countries, such as India and China, two of the world largest growing economies, have ratified the Protocol and are taking steps required to reduce their emissions, although not being legally required to do so, as both these countries are part of Annex II (Montgomery, et al, 2006).

There is widespread criticism of the Kyoto Protocol, in regard to the commitment needed to stabilise the climate system (Golub, et al, 2006). The impact of the Protocol's first commitment period emission reductions relative to 'BAU' emission projections is likely to be limited. Even if 
the target of the Protocol is met, reducing Annex 1 emissions to 5 percent below 1990 levels, it is now clear that the Protocol's impact on concentration of GHGs would be marginal.

However, the Kyoto Protocol was designed as an initial stepping stone and was, in itself, not intended to prevent anthropogenic climatic change but simply to take the first step in doing so. Another factor explaining why the Protocol is limited in stabilising GHGs concentrations in the atmosphere is that in its development in the mid-nineties, the science of climate change was less clear and there was intense opposition from countries such as Australia and the USA to ambitious emission reduction targets.

Annex I countries can meet their targets through domestic climate change policies and the use of Kyoto Mechanisms. There are three Kyoto Mechanisms:

\section{Joint Implementation (JI)}

Under Article 6 of the Kyoto Protocol, an Annex I Party may implement a project that reduces 'BAU' emissions or enhances removals by sinks in the territory of another Annex I Party and count the resulting emissions towards meeting its own target.

\section{Clean Development Mechanism (CDM)}

Under Article 12 of the Protocol, the Clean Development Mechanism allows Annex I countries to invest in projects that reduce emissions in developing countries, such as India and China, as a cheaper alternative to investing in emission reductions in their own countries (UNFCCC, 1998). Without the incentives provided by the Kyoto Protocol, such projects would not have occurred. Due to costs of emission reduction generally being far lower in Annex II countries, the CDM allows a given net global emissions reduction to be achieved at lower global cost.

\section{Emission Trading}

Article 17 of the Kyoto Protocol allows Annex I Parties to acquire emission reduction units from other Annex I Parties that have already satisfied their Kyoto obligations and use them towards meeting their Kyoto targets. Also known as the Kyoto compliance market, this emission market allows Annex I Parties to reduce the overall cost of reducing emissions, as it encourages greater 
emission reductions where marginal costs are lowest. A unit is equal to one metric tonne of $\mathrm{CO}_{2}$ equivalent $\left(\mathrm{CO}_{2} \mathrm{e}\right)$. Under the Protocol, units that can be traded include AAUs - assigned amount units; ERUs - emission reduction units arising from Joint Implementation Projects, or CERs Certified Emission Reduction units arising from the CDM.

\section{The Voluntary Carbon Market}

Beyond the three Kyoto Protocol mechanisms and the Kyoto Protocol market, another carbon trading market, known as the voluntary carbon market, exists. Companies, governments, non governmental organisations, or individuals can purchase carbon credits for purposes other than meeting Kyoto targets. Voluntary carbon credits cannot be used in the Kyoto market. By investing in carbon reduction projects, companies and organisations can on-sell these emission reductions to consumers. Consumers can then offset their own personal GHG emissions. Between 2005 and 2006, trade in the global voluntary carbon market tripled. This generated 24 million tonnes of voluntary offsets (Ward, et al, 2007).

\section{Voluntary Retail Market Source}

Offset projects can be classified into two generic categories (Figure 2):

1. $\mathrm{CDM} / \mathrm{JI}$ - projects that involve CERs and ERUs

2. Non-CDM/JI - verified projects that do not fall under the Kyoto emission reduction mechanisms. These are known as Verified Emission Reductions VERs.

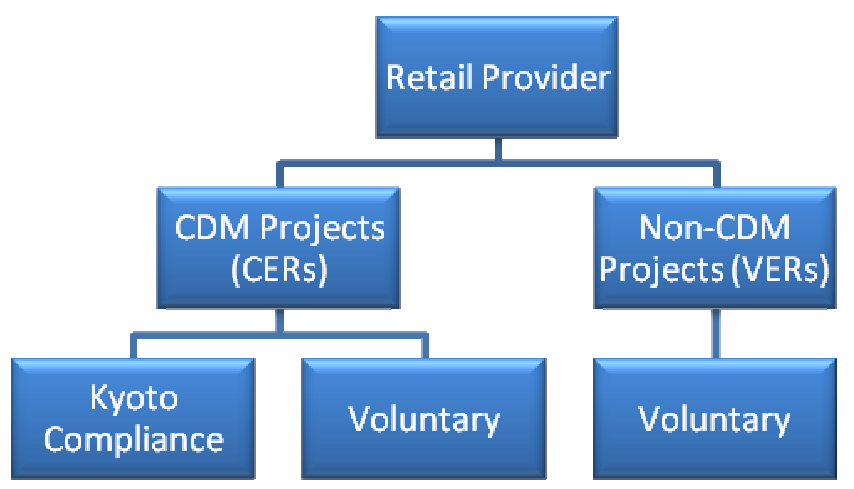

Figure 2: Schematic of the Voluntary Retail Market (Based on: Taiyab, 2006: 8) 
Carbon credits can be purchased from a CDM or a non-CDM project. As long as these credits are not used for meeting regulatory targets, they can be sold on the voluntary market. VERs, CERs or ERUs can be used for either the Kyoto Compliance market of the voluntary market, but the majority of credits sold on the voluntary market are from VERs (Taiyab, 2006). Kyoto credits are more valuable and expensive than equivalent credits purchased from non-Kyoto projects, so there is little incentive to use Kyoto credits in the voluntary market.

\subsubsection{Significant issues arising from the carbon market}

The following paragraphs identify some of the significant analytical issues relating to the integrity of the carbon market. These are relevant to the choices any institution, such as VUW, or individual faces when deciding whether and how to become carbon neutral.

\section{Additionality}

Carbon credits can only be counted if reduction in emissions is additional to any that would occur in the absence of the certified project activity. For example, carbon credits obtained from the planting of a forest can only be counted if the forest was not planned to be planted anyway. The project's intent must be emission reductions; they cannot be 'BAU' projects. To ensure the integrity of both the Kyoto compliance market and the voluntary market, the concept of additionality must be adhered to (Ward, et al, 2007).

\section{Double counting}

Another key concern associated with carbon credits, potentially compromising credibility, is that of double counting of emission reductions. This occurs when emission reductions are claimed by two or more parties. Buyers and consumers must be wary of this when purchasing credits on the voluntary market, as rogue retailers may sell the same carbon credits to another party. The Kyoto protocol market has a credit registration system that, if working properly, eliminates the risk of double counting. 


\section{Voluntary standards and industry guidelines}

To counter the significant issues mentioned above, there have been various standards and guidelines established. These have been developed by various groups, such as governments, individuals and organisations. These standards and guidelines include (Ward, et al, 2007):

Gold Standard for Voluntary Emission Reductions - this is the most rigorous standard to date. It only supports renewable energy projects that have proven additionally, which benefits local communities. It does not support land-use, land-use change or forestry projects.

Voluntary Carbon Standards - This standard provides a 'robust, new global standard and programme for approval of credible voluntary offsets'. In order to be a VCS offset, a carbon credit must be real, additional, measurable, permanent, independently verified, and unique, thus meaning that it can not be sold to two or more parties. VCS was initiated by The Climate Group, the International Emissions Trading Association and the World Economic Forum in 2005. Two versions of the VCS were released for submissions during 2006 (VCS, 2007).

\section{Accreditation of verifiers/certifiers}

Standards and guidelines cannot guarantee the quality of offsets. Validation and verification by qualified, independent third party auditors will provide a sense of creditability for investors. In 2006 the International Organisation for Standardisation (ISO) introduced ISO standards to be used as templates for voluntary offset projects. To prevent double counting, electronic registry systems have been set up, similar to the Kyoto compliance system, for instance, in New Zealand, the "TZ1" carbon trading platform is being developed (Ward, et al, 2007).

\subsection{New Zealand's Emission Reduction Response}

\subsubsection{A Brief History}

New Zealand played a key role in negotiations leading up to the adoption of the Kyoto Protocol in 1997 (Boston, 2007). The New Zealand Government ratified the Kyoto Protocol in December 2002. By ratifying the Protocol the Government has agreed to reduce the country's GHGs during 
the first commitment period $(2008-2012)$ to their Kyoto Protocol target, which is equivalent to their 1990 level of emissions (Renowden, 2007).

Since the late 1990s gross emissions (before accounting for forest sinks) have continued to rise, and in 2006 they were 20 percent above the 1990 baseline (Boston, 2007). From late 1997 to 2005, New Zealand's mitigation response was minimal and out of step with the seriousness of the emerging climate change problem (Chapman and Boston, 2007). However, since ratifying the Kyoto Protocol the government has analysed and developed options to begin reducing the rate of growth of emissions. To date, some of these measures have been implemented.

The Government first announced its intention to introduce a carbon tax in 2002 to meet the country's Kyoto commitments to reduce GHG emissions. This tax was to apply across the economy, excluding agricultural methane and nitrous oxide emissions. In early 2005 the Government released details of the carbon tax, setting a price of $\$ 15$ per tonne of $\mathrm{CO}_{2}$-equivalent. The tax was scheduled to take effect from April 2007 (Hodgson, 2005). In late 2005, however, the Government announced that it would not proceed with the carbon tax and would instead consider other options to ensure New Zealand meets its commitments (Parker, 2005)

In her 2007 annual speech to Parliament, the Right Honourable Prime Minister Helen Clark announced the ambitious goal of New Zealand becoming the first carbon neutral country across all sectors (Clark, 2007). The likelihood of reaching this goal is open to debate, given the government's record of action on climate change to date (Chapman and Boston, 2007).

However, the New Zealand government is beginning to lead by example. To this end it is aiming to make the public sector carbon neutral. The first group of six departments announced their commitment to achieving carbon neutrality by 2012. In early 2008, the Ministries for the Environment, Health, and Economic Development, the Departments of Inland Revenue and Conservation, and Treasury are working to significantly reduce their carbon footprint. The primary objective is to reduce each department's emissions, followed by offsetting emissions that cannot be further reduced.

Over the next few years all other government departments will develop and implement plans to reduce their emissions. By 2012, all public service departments are required to commit to 
becoming carbon neutral. The wider state sector will also be encouraged to join this programme (Clark, 2007).

The New Zealand government recently unveiled a framework for GHG reductions across all sectors, alongside plans to enhance carbon sinks in the forest sector through the adoption of an Emissions Trading Scheme (ETS). Operating within the Kyoto Protocol, the ETS is a price-based mechanism that aims to influence a wide range of firms and consumers, covering all major sectors of the economy (forestry, transport, stationary energy, industrial processes, agricultural and waste) by 2013 (NZ Government, 2007).

The ETS will obligate these sectors to hold emission units that match the emission levels for which they are responsible. A finite number of emission units will be issued in New Zealand each year and the scheme will operate within a global cap on emissions set by the Kyoto Protocol (NZ Government, 2007: 5). Through this scheme, the New Zealand government, rather than concentrate on actual emission reductions, aims to reduce its financial liability for national emissions above New Zealand's allocation of emission units' equivalent to 1990 levels.

In June 2007, the Wellington City Council (WCC) voted on a plan that will see the city embark on a path towards becoming carbon neutral. Though the Council has already committed to a plan of action with Communities for Climate Protection (CCP-NZ), it will now develop specific mechanisms such as provision of options facilitating carbon emission reductions and offsetting measures. In addition, the Council committed to developing infrastructure and urban development that is aligned with the move towards carbon neutrality. If these goals are achieved, this ambitious vision will see Wellington city become the first capital city in the world to become carbon neutral. Mayor Kerry Prendergast views this move as a 'need to future-proof the city against the environmental, social and economic impacts of climate change' (WCC, 2007a).

Following this declaration, in December 2007 Wellington City Councillors agreed to adopt a Climate Change Action Plan that involves achieving carbon neutrality for its own operations by 2012. This is, by any standard, an ambitious plan, and a bold signal of leadership for the wider Wellington community. The WCC's target is a reduction in emissions of 30 percent below 2001 levels by 2020 and 50 percent below 2001 levels by 2050 (WCC, 2007b). 


\subsection{Carbon Neutrality}

The following section focuses on defining carbon neutrality and outlining issues surrounding the concept. The section will conclude with the criticisms of carbon neutrality.

\subsubsection{Definition of carbon neutrality}

There is no single definition of carbon neutrality (Ward, et al, 2007: 3). However, the New Oxford American Dictionary declared it as the word(s) of the year in 2006. It was defined as: (McKean, 2006: 257)

\footnotetext{
"Being carbon neutral involves calculating your total climate-damaging carbon emissions, reducing them where possible, and then balancing your remaining emissions, often by purchasing a carbon offset: paying to plant new trees or investing in "green" technologies such as solar and wind power"
}

Carbon neutrality is not a static state but an engaged process. Simply stated, carbon neutrality involves indemnifying against a quantity of GHG emissions via an additional activity that removes an equal quantity of GHG from the atmosphere or by preventing this quantity from being emitted by others activities.

\subsubsection{Concept of carbon neutrality}

The concept of carbon neutrality can be explained by examining the dynamic balance that describes a compound's atmospheric concentration. A change in atmospheric concentration of any compound $(\Delta \mathrm{C})$ is described by six variables as follows:

$$
\Delta \mathrm{C}=\mathrm{T}_{\text {in }}+\mathrm{P}+\text { Sources }-\left(\mathrm{T}_{\text {out }}+\mathrm{L}+\text { Sinks }\right)
$$

Equation 1 (Isham, et al, 2003: 17)

Where:

- $\mathrm{T}_{\mathrm{in}}$ is the transport of that compound into a specific region of the atmosphere;

- $\quad \mathrm{P}$ is chemical production of that compound in the atmosphere;

- Sources are emissions of the compound into the atmosphere;

- $\mathrm{T}_{\text {out }}$ is the transport of that compound out of that region;

- $\quad \mathrm{L}$ is chemical loss;

- Sinks are removal processes of the compound's removal from the atmosphere (Isham, et al, 2003: 17). 
This equation can be greatly simplified for $\mathrm{CO}_{2}$ as it is one of the most important and long-lived anthropogenic GHGs in the atmosphere, accounting for more than half of human-induced radiative forcing (IPCCa, 2001). Because $\mathrm{CO}_{2}$ is uniformly mixed in the lower atmosphere, the sum of the transport in and transport out terms becomes negligible: $\mathrm{T}_{\mathrm{in}} \approx \mathrm{T}_{\text {out }}$. Similarly, the chemical production and chemical loss of $\mathrm{CO}_{2}$ within the atmosphere are relatively balanced processes and tend to be slow in comparison to the activity of sources and sinks (Isham, et al, 2003: 17). Therefore, for $\mathrm{CO}_{2}$ the above equation can be simplified to two terms (Jacob, 1999, Isham, et al, 2003):

$$
\Delta \mathrm{CO}_{2}=\text { Sources }- \text { Sinks }
$$

Equation 2 (Williams, 2001: 236)

\subsection{GHG Sinks and Sources}

There are two ways in which the destabilisation of the global carbon cycle can be linked to the increase in the Earth's population and human activity. The first is an increase in the burning of fossil fuels and wood for energy, releasing vast amounts of GHGs into the atmosphere. The second is the large-scale depletion of vegetation, leaving fewer sinks to remove, through the process of photosynthesis, GHGs such as $\mathrm{CO}_{2}$ (Williams, 2001).

A GHG source is defined by the UNFCCC (1992) as any process or activity that releases a GHG, aerosol $^{2}$, or precursor ${ }^{3}$ of a GHG into the atmosphere. A GHG sink is defined by the UNFCCC as any process, activity or mechanism that removes a GHG, aerosol, or precursor of a GHG from the atmosphere.

Likewise, in the GHG Protocol Initiative ${ }^{4}$, a GHG source is any process that emits GHG emissions into the atmosphere. There are five defined GHG source categories:

- Combustion emissions from generating grid-connected electricity.

\footnotetext{
${ }^{2}$ Aerosols are a collection of air-borne solids of liquids, with a typical size between $0.01 \mu$ and $10 \mu$ that reside in the atmosphere for at least several hours.

${ }^{3}$ Precursors are atmospheric compounds that are not GHGs or aerosols, but that have an effect on GHG or aerosol concentration by taking part in physical or chemical processes regulating their production or destruction rates.

${ }^{4}$ The Greenhouse Gas Protocol Initiative was designed in conjunction with both the World Resource Institute (WRI) and the World Business Council on Sustainable Development (WBCSD). Further detail will be given in Chapter 3. The GHG Protocol Initiative is used to define sources and sink, because the URS Environmental Audit used the GHG Protocol Initiative as the basis for their auditing process (URS, 2007).
} 
- Combustion emissions from generating energy or off-grid electricity, or from flaring.

- Industrial process emissions - e.g. cement production.

- Fugitive emissions - e.g. GHG leaks from pipelines.

- Waste emissions - e.g. GHG emissions from landfills.

Under the GHG Protocol Initiative, a GHG sink is any process that removes and stores GHG emissions from the atmosphere. The GHG Protocol Initiative defines only one GHG sink category:

- Increased storage or removal of $\mathrm{CO}_{2}$ by biological storage.

Although not classified as a GHG sink by the GHG Protocol Initiative, carbon sequestration is another GHG sink. Carbon sequestration involves capturing and storing $\mathrm{CO}_{2}$ sourced from the combustion of fossil fuels, as in power generation, or from the preparation of fossil fuels, as in natural gas processing. Capturing $\mathrm{CO}_{\mathrm{s}}$ involves separating $\mathrm{CO}_{2}$ from other gases and storing it sites, such as oil wells, where it can be stored for a very long time. This process can also be applied to certain industrial processes (IPCC, 2005).

\subsection{GHG concentrations in the atmosphere}

In order for the atmospheric change in concentration of $\mathrm{CO}_{2}$ to equal zero, two scenarios are available:

1. Decrease the sources of $\mathrm{CO}_{2}$ until they equalise with the existing sinks, or

2. Increase the sinks of $\mathrm{CO}_{2}$ until they equalise with existing with sources.

The target of both these scenarios is to have the change in atmospheric concentration of $\mathrm{CO}_{2}$ $\left(\Delta \mathrm{CO}_{2}\right)$ equal zero. In order to input no additional $\mathrm{CO}_{2}$ into the atmosphere and thus mitigate further anthropogenic climate change, the sources and sinks of $\mathrm{CO}_{2}$ must be equal. As it takes time for this equilibrium to be reached, if only the first scenario is employed to balanced concentrations, the concentration will be larger than today's atmospheric concentration. A similar situation would eventuate if only the second scenario were to be employed. To actually reduce the current levels of $\mathrm{CO}_{2}$ in the atmosphere, the total number of sources must be fewer than the total number of sinks. 
These are the basic premises of carbon neutrality. Having discussed the concept of carbon neutrality and placed it within the context of climate change science and of New Zealand's response to climate change, it is now possible to turn to the subject of this thesis, which is carbon neutrality at VUW.

\subsection{Rationale for carbon neutrality at VUW}

Perhaps the most basic answer as to why VUW should become carbon neutral - an answer which is consistent with arguments made by advocates of corporate social responsibility literature (CSR) discussed in the next chapter - is given by Creighton (1988: 6):

\footnotetext{
"Since universities are generally long-lived institutions, they should be concerned with the long-term health and liveability of their community and regions."
}

Shriberg (2002), addressing the question as to why universities might become sustainable, outlines five key points that also apply to becoming carbon neutral:

\section{1) Expertise / Ability}

Universities have the expertise, leverage and resources to advance progress on mitigating climate change. They also have less fiscal pressure than other large institutions and have the ability to act on vision. Universities should lead society, not because they are qualified for the role, but because they are the only institution with the ability to lead (Shriberg, 2002). Universities have a unique academic freedom and the critical mass and diversity of skills to develop new ideas. They are able to comment on society and the challenges society faces and so help create a healthy, just and sustainable world (Cortese, 2003).

\section{2) Social/ Ethical obligation}

Universities have a unique role, receive special benefits and are held in high regard within society. At an ethical level, the case for such an organisation to act to mitigate climate change is strong (Pierrehumbert, 2006). Pragmatically, any single institution's impact is limited; on the other hand, high profile institutions acting early outside government can demonstrate leadership. 
Universities are often at the forefront of teaching and research on climate change. The threat that climate change represents is well known within the academic community. To teach about climate change and then to do nothing constitutes hypocrisy from an ethical perspective. GHG emissions caused by institutions such as universities in the form of energy, transport, waste and material consumption have the potential to be considerably reduced by effective organisational and technical measures.

By moving to zero net GHG emissions, a university can avoid adding to the world's total GHG emissions and, importantly, help persuade like-minded organisations to act in such a manner. Thus the university can demonstrate both environmental integrity and assume a leadership role in this concern.

\section{3) Role Models}

The way in which universities conduct their operations will serve as a role model to students and society in general (Nicolaides, 2006). By leading the way, universities are able to direct society towards a more sustainable way of living. 'Even as universities teach their students that the vital signs of the planet are in decline, graduates leave university to begin lives that generally contribute to, rather than mitigate, a growing array of environmental and social problems' (Uhl and Anderson, 2001: 36).

\section{4) Problem-causers}

It is claimed the present problems that society faces are reinforced by universities in two ways. First, and most importantly, many graduate students leaving universities lack environmental knowledge and are leading society down an unsustainable path (Orr, 1994, Shriberg, 2002). A major shortcoming of the teaching philosophy of universities is the belief that they exist solely to educate within specialised disciplines. To wit, universities are divided into faculties: science, arts, commerce, law; and are then further divided up into schools and departments: such as chemistry, physics and biology. Despite evidence showing that there is a current planetary crisis; many students are not taught to think holistically and to perceive the interconnectedness of the systems they are a part of. "Ultimately, then, the ecological crisis concerns how we think and the institutions that purport to shape and refine the capacity to think" (Orr, 1994: 2) 
The second way that universities reinforce the ecological problems society faces is that universities, far from being isolated ivory towers, are part of society as a whole - they impact upon the environment and should therefore make the reduction of this environmental impact a top priority (Creighton, 1998). This will be expanded on in the next chapter.

\section{5) Image Benefits}

Within carbon neutrality, there exists the potential to positively influence an institution's image amongst stakeholders. Universities can use environmental efforts as a selling point to prospective students and to the community (Creighton, 1998). The demand amongst students for environmental education and for institutions to reduce their environmental impact is growing (Cortese, 1999). Universities are highly sensitive towards environmental programmes in peer institutions and thus strive to maintain a positive image by emulating these programmes (Shriberg, 2002).

If the university decided to embrace carbon neutrality, there would be additional short-term operational cost. However, at a societal scale, the cost of doing nothing may be far greater. The Stern Review found that:

\footnotetext{
"Using the results from formal economic models, the Review estimates that if we don't act, the overall costs and risks of climate change will be equivalent to losing at least 5\% of global GDP each year, now and forever. If a wider range of risks and impacts is taken into account, the estimates of damage could rise to $20 \%$ of GDP or more. In contrast, the cost of action - reducing greenhouse gas emissions to avoid the worst impacts of climate change - can be limited to around 1\% of global GDP each year" (Stern, 2006, vi).
}

By reducing emissions, the university can not only demonstrate leadership, but also play its part in reducing the costs to the planet. Clearly, the university could continue with 'BAU' emissions, free riding on the actions of other organisations (Tietenberg, 2003: 75), in the hopes these organisations will act to abate the high costs of climate change, as noted by the Stern Review.

\subsection{Criticism of Carbon Neutrality}

While the aim of this thesis is to investigate whether and how VUW can become carbon neutral, the author is aware that there are various critics of carbon neutrality. Carbon Trade Watch, a project of the Amsterdam-based think tank the Transnational Institute, produced a report in early 
2007 entitled "The Carbon Neutral Myth, Offset Indulgences for your Climate Sins" (Smith, 2007).

The central criticism targets the prevailing emphasis on carbon offsets. Buying offsets can allow organisations to maintain 'BAU' GHG emissions or even increase their GHG emissions, while remaining guilt-free by investing only in offsetting (Smith, 2007; Ball, Hughes and Milne, 2007). Carbon Trade Watch argues that offsets place too much attention on individual behaviour and carbon footprints, as opposed to systemic changes and urgent political will to properly tackle climate change (Smith, 2007).

A more telling criticism is that the carbon offset market is often unregulated and secretive, which can lead to deception and fraud. There are two types of offset: the official offsets sanctioned under the auspices of the Kyoto Protocol, and unofficial offsets, whereby charitable and profitmaking organisations charge a fee to offset emissions that are not counted in the official national inventory. Unofficial offsets cannot confer Kyoto credits and are not bound by the Protocol's rules. Careful consideration has to be given when investing in offsetting initiatives (Pearce, 2007). Investment in offsets that meet standards and industrial guidelines, which are verified, can negate this concern.

While these criticisms may be in part be true, many carbon neutrality initiatives do not centre solely on emissions accounting and offsetting, but also include energy efficiency and emission reductions. Although an entire systemic change may be needed to deal with climate change, an important first step is the transition towards reducing the GHG concentration in the atmosphere. For this to happen, it is important that organisations such as VUW account for their emissions, and then begin to reduce them.

An important argument for carbon neutrality, and in defence of carbon offsetting in particular, is the need to reduce carbon emission sources and increase carbon sinks. Carbon offsets can help achieve both. Planting trees can help increase the number of available carbon sinks while project-based carbon offsets, such as renewable energy projects, help reduce carbon sources. As discussed above, both of these actions will result in a net reduction of the GHGs in the atmosphere. Focusing only on emission reductions will mean that concentrations of GHG in the atmosphere will be higher in the future than at present, as net GHG emissions are still positive. 


\subsection{Summary}

Scientists have discovered that anthropogenic GHG emissions are changing the planet's climatic system. The effects of this are potentially catastrophic for the environment, the economy and on society. Although there have been both international and domestic mechanisms developed to counter the increasing GHG emissions in the atmosphere, emissions are still increasing. Both the New Zealand government and the Wellington City Council have signalled their intent to become carbon neutral as a response to climate change. Carbon neutrality involves balancing sources of emissions with available sinks, resulting in net emissions equalling zero. Although there are several rationales for an organisation such as VUW to become carbon neutral, carbon neutrality can be criticised, depending on how it is carried out. 


\section{The University as an Agent of Change and the CSR model}

This chapter begins with discussing universities as research and teaching institutions and agents of change. The next section discusses the CSR model and its application to universities. It then goes on to discuss the CSR model, and its applications in detail.

\subsection{Universities as agents of change}

Universities have been instrumental in identifying and developing strategies to mitigate the growing ecological crisis facing humanity. Faculty, students, and others involved in higher education are influential in the environmental movement in New Zealand (Chapman, et al, 2006). The potential of universities to take a lead role in guiding society through the current climatic crisis has been recognised by the United Nations. The Agenda 21, Chapter 36.1 (i) (UNCED, 1992) recommends that:

\footnotetext{
“i) Countries could support university and other tertiary activities and networks for environmental and development education. Cross-disciplinary courses could be made available to all students. Existing regional networks and activities and national university actions which promote research and common teaching approaches on sustainable development should be built upon, and new partnerships and bridges created with the business and other independent sectors, as well as with all countries for technology, knowhow, and knowledge exchange".
}

Yet, there is often reluctance amongst universities to make the environmental and interconnected multidisciplinary sustainable development issues a top priority in its teachings, research and operations (Shriberg, 2002). As Creighton points out (1998: 12):

\footnotetext{
"Universities and colleges that have existed as institutions for many years have well-established systems of operating and they tend to be fiscally and operationally conservative. Moreover, they are purveyors of ideas and the teachers of classic methods, history, and a body of knowledge that is often more theoretical than practical, making these institutions less than adept at the pragmatic thinking needed for implementing campus environmental action”.
}

This can be explained in part by the traditional role of academic governance dating back to the Middle Ages (Altbach, 1972: 2). With little variation, the university community is made up of five groups: the University Council, the administration, the faculty, staff and students. A sixth 
agent, the surrounding community, is typically considered an external stakeholder rather than a part of the university family (Creighton, 1998).

Universities' response to climate change thus far has often contradicted their role as employers of those at the forefront of change, including pioneers in thinking about climate change science and policy. Empirical studies have suggested a variety of reasons for why universities may be reluctant to actively implement appropriate environmental action at their campuses (for example, see, Smith, 1993, Creighton, 1998, Dahle and Nauemayer, 2001; Wright, 2005). These include internal barriers to organisational change, misunderstanding the meaning of sustainability, bureaucratic inertia, resistant standard operating procedures, risk aversion, lack of vision and leadership from staff and students, and narrow definitions of costs and benefits in implementing 'green initiatives' (Breyman, 1999; Dahle and Nauemayer, 2001).

When change does occur in how a university functions, it often "comes from a result of careful and usually time-consuming deliberation by official committees" (Altbach, 1974: 1). According to Shriberg, the official committee-based process of organisational change stems from the academic world (2002: 30). However, a university committee can be an effective means to progress, provide an opportunity for the wider university community to raise issues and learn about where the university is heading (Creighton, 1998).

\subsection{Corporate Social Responsibility and its application at Universities}

The combined effects of legal and regulatory pressure, market and non-market incentives and growing public support promote the improvement of organisational environmental performance. The very nature of a university with its academic freedom, tenure, governance, adjunct and parttime teaching, tensions between teaching and research, and the student life-style, makes it quite distinct from the business world (Walton and Galea, 2005). However, several studies have drawn on business models, such as the 'corporate social responsibility' model to explain how universities might become more sustainable (see: Levy and Dilwali, 2000; Comm and Mathaisel, 2003; Fisher, 2003; Walton and Gale, 2005). Universities involve the various members of the university community to partake in the development of environmental initiatives. 


\subsection{Historical Background of CSR}

Over the last few decades, corporate social responsibility (CSR) has evolved from a marginalised theory into a diverse and complex practice, being increasingly pivotal to many of today's business decisions (Cruz, 2006; Cochran, 2007). CSR is used to justify an organisation's business practices to key stakeholders and society in general (Ingenbleek, et al, 2007). The definition of CSR is not always clear. The World Business Council for Sustainable Development offers one definition (1999: 3):

\footnotetext{
CSR is "the ethical behaviour of a company towards society. In particular, this means management acting responsibly in its relationships with other stakeholders who have a legitimate interest in the business - not just the shareholders... CSR is the continuing commitment by business to behave ethically and contribute to economic development while improving the quality of life of the workforce and their families as well as of the local community and society at large."
}

CSR is gaining importance in today's business world. Over 90 percent of the US-American Fortune 500 companies had CSR initiatives in place by 2004 (Malovics, et al, 2007). Often companies back up their social responsibility credentials by producing substantial papers, or webbased environmental reports, and more recently, social and sustainability reports (Cooper and Owen, 2007).

An example of a corporation demonstrating its social responsibility and environmental performance is United Parcel Service (UPS). UPS formed a partnership with two NGOs, the Environmental Defense Fund and the Pew Charitable Trust to redesign their Next Day Air packaging. The result was packaging that is both lighter and reusable. UPS estimated that this saves them about US\$1.6 million a year, eliminated 550 tonnes of solid waste and saves 2,200 tonnes of trees. Other examples are DuPont, Ricoh, Toyota, Honda, Intel, The Gap, The Body Shop, and BMW. Corporate concern for the environment has become an issue for executive leadership and it is seen that companies are beginning to respond (Walton and Gale, 2005).

However, historically, the discourse on CSR has viewed environmental issues as one of many social issues that organisations face, along with issues of class, race, gender, business ethics, community welfare and needs, minority concerns and stakeholder demands, to name a few 
(Shrivastava, 1995). In a broad sense, all these issues are interconnected in terms of business sustainability and a business's licence to operate.

A neo-classical view of business would have the provision of employment and payment of taxes as an organisation's only social responsibilities. This is reflected in the views of Milton Friedman (1962: 133; cited in Moir, 2001):

\footnotetext{
"Few trends would so undermine the very foundation of our free society as the acceptance by corporate officials of a social responsibility other than to make as much money for their shareholders as they possibly can."
}

Critics of CSR claim that it relies on 'dubious or false assumptions', and that CSR advocates are adherents of 'global salvationism', offering an alarmist view on the state of the environment and the damage done to it by business-related activities. They believe the adoption of CSR will impose unnecessary costs on businesses, lowering profits and reducing employment. As such, organisations adopting CSR are disadvantaged relative to organisations less socially and environmentally responsible (Aupperle, et al 1985; Ullmann, 1985; Henderson, 2001).

This can be refuted. For example, as discussed further in Chapter 4, universities that account for their emissions and have conducted emissions audits predict long-term economic gains rather than losses. The market-driven viewpoint offered by critics of CSR neglects to mention the numerous market failures that can occur in a pure market system where profit is the bottom line. The fundamental problem with this viewpoint is that there is no price for non-market goods such as environmental amenities and climate services.

There is also evidence that the more sustainable oriented companies perform as well as, if not better, than companies with a narrower focused. The Dow Jones Sustainability Index is the first global index tracking the financial performance of the leading companies in terms of sustainability around the world. It captures more than 300 companies that represent the top $10 \%$ based on long-term economic, environmental and social criteria (DJSI, 2008). 


\subsection{Benefits, Opportunities, and Barriers of CSR}

Without a clear-cut understanding of the strategic benefits and opportunities that emerge from CSR practice, as well as the barriers to such practices' implementations, it will be difficult to convince top-level managers to engage - and more importantly invest - in any such programmes. VUW will need to identify these benefits, opportunities and barriers in order to successfully promote and implement a carbon neutral programme (Dahle and Neumayer, 2001; Thompson and Green, 2005).

The following subsection defines both the 'enlightened self-interest' rationale and 'short-term interest' rationale as justifications for CSR. The opportunities arising from CSR initiatives, including carbon neutrality, can be established from these rationales. The final subsection discusses the barriers to the institution and development of CSR initiatives.

\subsubsection{Enlightened self-interest rationale}

CSR theory and practice are beginning to focus on enlightened self-interest as a factor for change (Balabanis et al, 1998; Shriberg, 2002). Enlightened self-interest prioritises long-term planning of an organisation's strategic positioning within society and the environment over short-term profitdriven goals. The benefits include an improved reputation leading to stakeholder engagement and greater employee loyalty and retention.

The demands and concerns of stakeholders are a key component in the enlightened self-interest rationale. Decision-making that responds to the needs of stakeholders is emphasised (Cooper and Owen, 2007). An important aspect of enlightened self-interest is the notion that businesses, communities, and the environment are interconnected. It follows that broad stakeholder engagement is vital for long term business survival.

Proponents of the enlightened self-interest rationale claim that organisations' positive engagement with stakeholders leads to benefits such as an improved reputation amongst employees and broader society (Moskowitz, 1972; Soloman \& Hanson, 1985). Studies have indicated that a positive external image leads to positive internal image. However, this link is even stronger when a business has a negative rather than positive external reputation; a greater negative external image can be more internally damaging (Shriberg, 2002). 
Another component of the enlightened self-interest rationale is an incorporation of ethics and values. Businesses are beginning to realise the importance of ethical practices, and in particular, values for the environment. This form of management can be regarded as 'value-driven environmentalism that focuses on intrinsic motivation as it relates to organisational culture, ethics, and image' (Post and Altman, 1994; Shriberg, 2002: 38).

\subsubsection{Short-term interest rationale}

The majority of literature on CSR stresses the importance of a short-term interest rationale. It follows the classic neo-classical economic theory of 'managerial decision-making', which favours myopia and extrinsic incentives (Shriberg, 2002). Implementing CSR initiatives can lead to a competitive advantage through economic gains from being part of the green market, increasing efficiency, and preceding government regulations (Porter and van der Linde, 1999).

However, this is highly debated. It is claimed that the short-term gains from CSR initiatives are exaggerated. Environmental initiatives that tend to be more expensive cannot be justified on the grounds of short-term profitability (Walley and Whitehead, 1994; Hart, 1995; Hussan, 1999). Gray et al (1993: cited in Hussan, 1999: 203) states: 'there is potential for rather more tension between environment and profit than there is congruence'. The link between CSR and short-term profit is tenuous at best.

Another short-term interest rationale for CSR is the avoidance of government regulation. An organisation that adopts CSR innovations may reduce risk, improve their relationship with government bodies and keep ahead of environmental regulation (Shrivastava and Hart, 1995). However, one study by Karagozoglu and Lindell (2000: 825) found that a progressive environmental strategy was a more important predictor of environmental innovativeness, independent of any favourable regulatory circumstances. The role of regulation is seen by the authors as more of an 'obstacle-reducing catalyser' than a triggering mechanism for an organisation's environmental performance.

\subsubsection{Barriers}

Overall, the literature suggests that the enlightened self-interest rationale is the strongest motivation for CSR. As universities are not profit-driven, the enlightened self-interest rationale 
can be strongly encouraged (Shriberg, 2002). Shriberg concludes that the strongest justification for campus environmental innovations is indeed an enlightened self-interest rationale, rather than short-term interest rationales (2002: 158). Successful environmental initiatives at universities are largely due to ethical and long-term strategic approaches.

The corporate environmental management and social responsibility literature outlines two basic types of barriers: industrial barriers that reflect the special and unique features of an activity in which an organisation engages; and organisational barriers that are not unique to environmental problems, but which affect an organisation's capacity to deal with any form of change (Post and Altman, 1994: 67).

Industrial barriers include lack of information, capital costs, competitive pressures and regulations, and configuration of current operations. Organisational barriers include factors such as attitudes amongst stakeholders, lack of communication and consultation, past practices, and lack of leadership from top management (Post and Altman, 1994: 67). These barriers to change are also present at universities.

The following is an example of an organisational barrier formed by the lack of leadership from top management. CEOs are accountable primarily to the company board and without direction from the board; there is little incentive to implement environmental initiatives (Stanwick and Stanwick, 1998). Often these initiatives are not pursued, as environmental projects tend to have short-term costs and long-term benefits.

Organisations such as universities are different to other institutions, as they are not accountable to shareholders but are instead accountable to a variety of stakeholders. Stakeholders include those individuals, groups, and other organisations that have an interest in the actions of an organisation and who possess the ability to influence the decisions that lead to these actions (Savage, at al, 1991). Similarly, Henriques and Sadorsky define stakeholders 'as any group or individual who can be affected by the achievement of an organisation's objective' (1999: 89). Stakeholders can influence an organisation by either direct pressure or by submission. .

The stakeholders that will be affected by VUW adopting a target of becoming carbon neutral and would need to be involved in decision-making include: the VUW Environmental Committee, the university council, including the vice-chancellor, faculty (including family), university staff, 
students (including family), local Maori, Gecko - the VUW Student Environmental Club, employers and others in the local business sector, the Wellington City Council, the Wellington public, and the government. Each stakeholder will have different levels of power, influence, and interest in the decision-making process.

\subsection{Organisational Environmental Reporting}

A component of CSR is the development of environmental reporting. Growing pressure from the public and internal pressure for change has led to an increase in organisations voluntarily collecting and releasing environmental information. The environmental information that organisations provide lies mainly outside the financial statements and contains both qualitative information and quantitative information on such areas as GHG emissions. One reason why reporting on environmental financial items is rare is that these items are not (yet) confined to accepted standard accounting conventions (Maltby, 1995, Nyquist, 2000).

The International Chamber of Commerce (ICC) defines environmental auditing as (ICC, 1991: 3):

\footnotetext{
"A management tool comprising a systematic, documented periodic and objective evaluation of how well environmental organisation, management and equipment are performing with the aim of helping to safeguard the environment by: (i) facilitating management control of environmental practices: and (ii) assessing compliance with company policies, which would include meeting regulatory requirements."
}

Environmental reporting is now well established, if not common in New Zealand. According to the ICC, environmental auditing began in the 1960s and then faded into the background, until resurfacing around 1989. The use of environmental auditing - both by practitioners and potential clients - had only become widespread in the United Kingdom (UK) and USA in the nineties (Malltby, 1995). In 1996, environmental auditing became part of the International Standards Organisation's (ISO) 14001 environmental standards, being renamed ISO 19011 in 2002 (Bardati, 2006).

As the sustainability of universities becomes more prevalent, there is the need to develop methodologies to measure a campus' level of sustainability. Environmental auditing is emerging as one potential approach (Conway, et al, 2008). Conducting an environmental audit can give an organisation a competitive advantage, differentiating itself within the marketplace, as the manner 
in which an organisation implements environmental management projects indicates how willing it is to fulfil its responsibilities to society (Walker, et al, 2007). The flexibility of an environmental audit allows it to be tailored to suit the specific organisation's situation and the meet the specific needs of the auditors designing the audit (Bardati, 2006).

On the other hand, voluntary reporting by organisations has been criticised, as it only seems to work if there is a large uptake of these voluntary measures, something that is not currently occurring in many countries. Also, environmental reporting tends to be shallow - rather than concentrating on an organisation's ecological footprint, reports are limited in scope, focusing on sustained economic growth. Furthermore, organisations may be vulnerable to accusations of green wash $^{5}$ (Onisto, 1999; Gray and Milne, 2004, 71:72).

Nevertheless, when an organisation begins conducting an environmental audit, this change of focus can redirect its attention towards becoming environmentally sustainable (Bardati, 2006). It is an information-gathering step and further developments can be made in implementing an environmental programme. This environmental programme can be planned from the initial audit. In this context, an environmental audit is an important initiative for VUW to take, as a critical first to achieving carbon neutrality.

\subsubsection{Carbon Auditing}

For organisations and institutions to address climate change, identify GHG opportunities, responsibilities and potential liabilities, they must first understand their carbon footprint - both its size and what influences its size. A GHG emissions inventory helps facilitate this understanding by identifying, quantifying and categorising major sources of emissions. Organisations that are committed to dealing with climate change and developing robust GHG reduction strategies will include a GHG inventory as an integral part of the reductions process (NZBCSD, 2002).

GHG emissions data are predominantly based on estimates due to the difficulty of measuring emissions directly and continuously. Emissions of non- $\mathrm{CO}_{2}$ gases from industrial processes may to some extent be directly measured, while emissions from other sources are normally estimates

\footnotetext{
${ }^{5}$ Green wash is used to describe the actions taken by companies to cover up their environmental wrongs by pretending to by environmental sound. Companies often spend more money or time advertising being green, rather than spending resources on environmentally sound practices
} 
based on activity data and emissions factors (emissions per unit activity). Data collated from each source is based on an assumption about the relationship between an activity and emissions generated (the emission estimation model) (Winiwarter and Rypdal, 2001).

According to the IPCC Guidelines for National GHG Inventories, to understand the effect a human activity has, as regards to GHG emissions, the most common simple methodological approach is to combine information on the extent to which a human activity takes place, called activity data or (AD) with coefficients which quantify the emissions or removals per unit activity. These are called emissions factors (EF). The basic equation is:

\section{Emissions $=\mathrm{AD} \times \mathrm{EF}$}

Equation 3 (IPCC, 2006: 6)

An emissions factor is a representative value that quantifies the amount of a specific GHG released into the atmosphere with an audit of activity associated with the release of that particular GHG. These factors are usually expressed as the weight of pollutant divided by a unit weight, volume, distance, or duration of the activity emitting the pollutant. In most cases, these factors are simply averages of all available data of acceptable quality and are generally assumed to be representative of long-term averages for all facilities in the source category (EPA, 1995). However, there is no standardised set of emission factors.

\section{Marginal Emission Factors}

In some cases, marginal factors are used instead of average emission factors. A marginal emissions factor is used to show how emissions change when the demand for electricity changes. There can be quite a large difference between marginal emission factors and average emission factors. Most of New Zealand's electricity comes from renewable sources; therefore the average emission factor is low, as it is calculated by dividing the total electricity emissions by total electricity production (Samuelson, et al. 2007).

The marginal emission factor is typically higher, as the assumption is made that renewable sources would be used first and fossil fuel-based electricity sources would be activated to meet extra demand as needed. Marginal emission factors are used to calculate savings, as they are an approximation of a worst-case scenario emission factor. For example, when using marginal 
emission factors for electricity, every $\mathrm{kWh}$ of electricity saved during times of peak load will reduce the emissions of $\mathrm{CO}_{2}$ produced by the most inefficient generation source e.g. coal and gas. However, marginal emission factors don't represent emissions produced on an average $\mathrm{kWh}$ basis (Samuelson, et al, 2007). New Zealand emission factors for electricity are discussed in Chapter 5.

\section{Errors in emissions data}

It is impossible to provide completely accurate emission data, including emissions that are directly measured from their source. There may be a range of reasons that result in uncertainties over an emissions inventory, e.g. errors in measurement instruments, bias in expert judgments, and natural variability of the emission-generating process (Monni, et al, 2004).

Numerous emission-producing activities are variable in space and time and it is difficult to develop estimation models and estimation data. Some processes may be poorly understood and may not be recognised as an emissions source. For other sources, estimation models may correctly record data but complete data may be missing to fill the models and guesswork may be needed. Finally, human error may result in inaccurate data collection (Winiwarter and Rypdal, 2001).

Inventory model errors are not distinguished within analysis. While most analyses use simple, linear formulas, this cannot represent the more complex generating processes of GHGs. Potential inventory model errors may also be linked to uncertainties in emission factors. This is a limiting factor of such analysis, due to lack of knowledge to carry out full analyses that include modelling errors (Rypdal and Flugsrud, 2001).

\subsubsection{Greenhouse Emission Guidelines}

In 2000, the United Nations Environmental Programme produced a guideline entitled The $G H G$ Indicator: UNEP Guidelines for Calculating Greenhouse Emissions for Businesses and NonGovernmental Organisations. This provided a step-by-step method for calculating emissions of different GHGs and then working out a single GHG footprint for an organisation. The guidelines were designed in collaboration with expert advice from manufacturing companies, accountants, academics, consultants, financial organisations, government departments and NGOs. One aim of the guidelines was to help countries meet their Kyoto commitments (Thomas, et al, 2000). 


\section{The GHG Protocol Initiative}

Another such guideline is the GHG Protocol Initiative. The protocol was designed in conjunction with both the World Resources Institute (WRI) and the World Business Council on Sustainable Development (WBCSD). It is an international, multi-stakeholder undertaking, aiming to promote the use of standardised methods for estimating and reporting business GHG emissions. The Initiative's intent is to lead to the adoption of a GHG reporting protocol that has a wide range of purposes, including helping companies identify GHG reduction methods and providing a tool for self-assessment or independent auditing (Vine, et al, 2003).

The GHG Protocol Initiative covers the accounting and reporting of GHGs covered within the Kyoto Protocol. These gases are: $\mathrm{CO}_{2}$, methane $\left(\mathrm{CH}_{4}\right)$, nitrous oxide $\left(\mathrm{N}_{2} \mathrm{O}\right)$, hydrofluorocarbons (HFCs), perfluorocarbons (PFCs), and sulphur hexafluoride $\left(\mathrm{SF}_{6}\right)$. The objectives of the GHG Protocol are (2004):

- To represent a true and fair account of an organisation's emissions, through a standardised approach;

- To simplify and reduce the costs of compiling a GHG inventory;

- To provide business with information that can be used to build an effective strategy to manage and reduce GHG emissions;

- To provide information that facilitates participation in voluntary and mandatory GHG programs;

- To increase consistency and transparency in GHG accounting and reporting among various companies and GHG programmes.

To properly manage GHG emissions, operational boundaries have to be defined, so that both direct and indirect emissions can be recorded. Direct GHG emissions are emissions from sources that are owned or controlled by an organisation. Indirect GHG emissions are emissions that result from an organisation's activities, but occur at sources owned or controlled by another organisation. Selecting an organisation's boundaries in terms of a consolidation approach (equity share of control), can be used to define how emissions are classified (The GHG Protocol, 2004) 
According to the World Resource Institute, three scopes (Scope I, Scope II and Scope III) are defined for GHG accounting and reporting purposes. To ensure consistent reporting and to prevent doubling up of two or more organisations reporting on the same emissions, both Scope I and Scope II are carefully defined. The GHG Protocol Initiative requires companies to account for and report on both Scopes I and II at a minimum. The Scopes are defined as (The GHG Protocol, 2004: 27):

- Scope I: Direct GHG emissions

Direct GHG emissions occur from sources that are owned or controlled by the organisation. For example, emissions from combustion in owned or controlled boilers, furnaces, vehicles and emissions from chemical production in owned or controlled process equipment.

- Scope II: Electricity indirect emissions

Accounts for GHG emissions from the generation of purchased electricity consumed by the organisation. Purchased electricity is defined as electricity that is purchased or otherwise brought into the organisational boundary. Scope II emissions physically occur at the facility where electricity is generated.

- Scope III: Other indirect GHG emissions

This scope includes all other indirect emissions. Scope III emissions are a consequence of the activities of the organisation, but occur from sources not directly controlled. Examples of Scope III emissions include extraction and production of purchased materials, transportation of purchased fuels, commuters travelling to and from the organisation and use of sold products and services.

The GHG protocol outlines a series of steps for organisations to follow. These steps include defining the organisational and operational boundaries, choosing a base year, tracking emissions over time, identifying and calculate GHG emissions for a given year, managing the GHG inventory, accounting for GHG reductions, reporting on these GHG reductions, verifying GHG emissions and setting GHG emission reduction targets. 


\section{The New Zealand Business Council for Sustainable Development}

The New Zealand Business Council for Sustainable Development and the Ministry of Economic Development produced a report in 2002 called "The challenge of greenhouse gas emissions: The "why" and "how" of accounting and reporting for GHG emissions". This report was an industry guide for measuring GHG emissions for New Zealand organisations and was adapted from the GHG Protocol Initiative. The guide is structured into three main sections (NZBCSD, 2002):

Why - This section provides a detailed reason why businesses account and report on their GHG emissions, including taking part in government initiatives, participating in GHG trading markets, understanding risk, identifying opportunities and cost savings and acting in accordance with government legislation.

How - Based on the GHG Protocol Initiative guide to emissions reporting, the NZBCSD's report provides a 10-step key to account and report GHG emissions in order to understand an organisation's GHG emissions, calculating these emissions into tonnes of $\mathrm{CO}_{2}$, and then reporting these emissions in line with international good practices (NZBCSD,2002).

Who - who should use the report and case studies of organisations that have already accounted and reported on their emissions in New Zealand (2000 -2003).

Six members of the New Zealand Business Council for Sustainable Development had volunteered to participate in this project by identifying business opportunities and report on their emissions. These members included BP Oil New Zealand Ltd, Hubbard Foods Ltd, Landcare Research, Meridian Energy Ltd, Milburn New Zealand Ltd, and Urgent Couriers Ltd (NZBCSD, 2002).

The NZBCSD accounts for the following source emissions for NZ businesses: petrol, diesel, LPG, gas, coal, electricity and air travel. It does not account for emissions that arise from waste or agriculture.

The NZBCSD website has emissions assessment tool, where users can input data based on the above source, from which a carbon footprint is then calculated. The web-based tool was used to 
calculate a 2006 GHG footprint for VUW, as a comparison to the VUW audit conducted by URS, result are in Chapter 5.

\section{Clean Air-Cool Planet}

Also based on the GHG Protocol Initiative, the Clean Air-Cool Planet Campus Calculator is a carbon emissions calculator tool used by over 200 higher learning institutions throughout North America (though not applicable to New Zealand universities). The on-line Campus Climate Action Toolkit (CCAT) allows institutions to not only calculate their $\mathrm{CO}_{2}$ emissions, but also for other GHGs specified in the Kyoto Protocol.

The tool provides a simple step-by-step guide for calculating emissions. Information on energy, agriculture, refrigerant and solid waste data can be entered into a MS Excel workbook and the tool provides a carbon footprint for any given year. Calculations can be made for years 1990 to 2020 (Clean Air-Cool Planet, 2000).

The tool calculator is the most recognised and widely used tool for calculating emissions for universities in North America, allowing institutions to conduct inventories and share information with each other on how to reduce GHG emissions. The American College and University Presidents Climate Commitment recommends its signatories to use the tool (ACUPCC, 2007). However, anyone can access the tool and member organisations can edit and format it on-line, therefore allowing it to be adapted to the needs of individual institutions.

\subsection{Summary}

Universities have been at the forefront of the teaching and research of climate change, yet, until recently, universities have often been slow at implementing appropriate on-campus emission reduction initiatives. Studies have indicated that a CSR model can be used to successfully implement environmental initiatives at universities. By using a CSR model the opportunities, benefits and barriers that exist in implementing an environmental initiative can be identified.

By adopting a CSR model, organisations can conduct an environmental audit to assess their environmental impact, including their GHG footprint. Several GHG emission guidelines have been developed to aid in this process. These include the GHG Protocol Initiative; the NZBCSD's 
reporting guide; and Clean Air-Cool Planet Campus Calculator - specially designed for the North American higher education sector. 


\section{GHG Emissions Reduction Initiatives at Universities Internationally}

This chapter focuses on objective one, investigating GHG emission reduction initiatives occurring at other universities, both in the USA and the UK. Several case studies are given.

\subsection{Universities and the status quo}

Universities have a vital role in tackling significant societal concerns and issues. As institutions of higher learning they are centres for intellectual inquiry, critical thinking, and innovation. Universities are there to educate members of society - including future leaders. Therefore it is imperative that they take the lead in the sustainability movement by increasing knowledge and public awareness surrounding environmental issues, by developing technology and motivating the drive for a sustainable future. Educational institutes' potential input in this area has been be understood by a variety of bodies, including the United Nations. The Agenda 21, Chapter 36 (UNCED, 1992: 36.1 - 36.27) recommends that:

\footnotetext{
"Countries should support university and other tertiary activities and networks for environmental and development education. Cross-disciplinary courses could be made available to all students. Existing regional networks and activities and national university actions which promote research and common teaching approaches on sustainable development should be built upon, and new partnerships and bridges created with the business and other independent sectors, as well as with all countries for technology, knowhow, and knowledge exchange."
}

Yet having the right curriculum for an environmentally literate society will not be adequate. Many educators and environmentalist believe that a university must, while teaching an ethic of responsibility, also demonstrate environmental responsibility in their wider activities (Pike, et al, 2003). Before universities can educate the wider community, they must be seen to 'walk the talk'

Universities can be seen as 'microcosms' of the problematic environmental scenarios facing humanity as a whole (Smith, 1993: 44). Consisting of a large community, universities consume a vast amount of energy, water, products, hazardous materials, fertilisers, and generate a variety of wastes and pollutants (Smith, 1993, xii). Furthermore, the commuting needs of the university can lead to congestion, noise, and air quality problems for local communities (Creighton, 1999: 4). 
As universities are not subject to the same constraints as other public and private organisations, they are able to take leadership roles in matters where the logic of the status quo can be questioned. In response to the risks associated with climate change, universities around the world have begun to address their energy use and GHG emissions (Marcell, et al, 2004). The following section will describe how some universities overseas are addressing this challenge.

\subsection{The United States of America}

\subsubsection{The American College and University Presidents Climate Commitment}

The American College and University Presidents Climate Commitment (ACUPCC) is a nonbinding pledge by the presidents of over 400 American colleges and universities aiming to make campuses more sustainable and to address climate change by establishing institutional commitments to reduce emissions and, ultimately, become carbon neutral. The premise behind the commitment was the recognition that higher learning institutions have to exhibit leadership in their communities and throughout society by modelling ways to eliminate global warming emissions, and providing the knowledge and the educated graduates to achieve climate neutrality. Campuses that address the climate challenge by eliminating GHG emissions and by integrating sustainability into their curriculum will help create a thriving, ethical and civil society. The group's goal is to have 1000 or more presidents sign the commitment by 2009 (Asquith, 2007).

The ACUPCC was developed in 2006 and is being coordinated and supported by Second Nature, ecoAmerica and the Association for the Advancement of Sustainability in Higher Education. The commitment provides a framework and support for institutions to become carbon neutral. By making a pledge to the commitment, institutions commit themselves to (ACUPCC, 2007):

- Establishing an institutional structure to oversee the development and implementation of the school's programme to comply with ACUPCC;

- Completing an emissions inventory within a year;

- Within two years, establishing a climate neutrality action plan and set a target date and interim milestone for becoming carbon neutral;

- Taking immediate steps to reduce GHG emissions by choosing two or more from a list of tangible action options while a more comprehensive plan is developed. These include: any construction of new buildings will be built to the U.S. Green Building Council's LEED 
Silver standard or equivalent; adopting an energy-efficient appliance purchasing policy; offsetting GHG emissions by any air travel paid for by the institution; develop a public transport travel plan; purchasing at least 15 percent of the institution's electricity consumption from renewable sources; supporting climate and sustainability shareholder proposals at companies where investments are made; or a comprehensive waste minimisation policy;

- Integrating sustainability into the curriculum and making it part of the educational experience; and

- Making their climate action plan, inventory and progress reports publicly available.

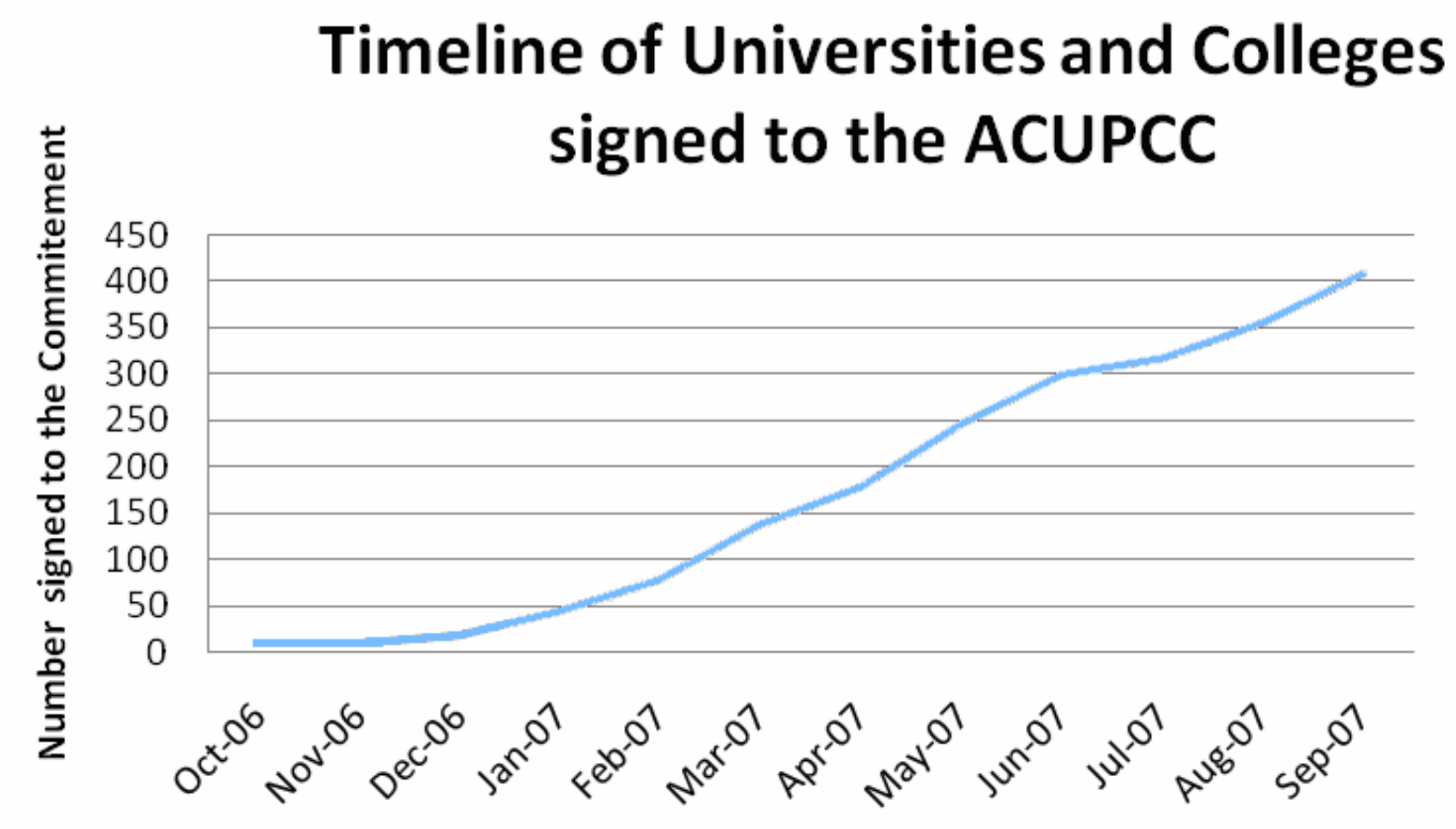

Figure 3: Timeline of Universities and Colleges signed to ACUPCC (pers. comm. Begley, 2007)

Figure 3 illustrates a rapid increase in the number of universities and colleges that are signing up to ACUPCC. In less than a year, over 411 higher learning institutions have agreed to the principle of becoming carbon neutral. It is to be noted that the commitment these institutions have signed extends beyond operational carbon neutrality to curriculum matters and transparency with regards their carbon neutral initiative. 


\subsubsection{The College Sustainability Report Card 2008}

The College Sustainability Report Card (CSRC) assesses and compares campuses throughout North American on the merits of their sustainability programmes. The report is produced by the Sustainable Endowments Institute, a Cambridge, Massachusetts-based non-profit organisation founded in 2005. Designed to identify colleges and universities that show leadership in sustainability, the CSRC provides a basis for North American higher learning institutions to share information by accessing the CSRC website. The report cards focus on policies and practices in eight different areas (Sustainable Endowments Institute, 2007):

- Administration

- Climate Change and Energy

- Food and Recycling

- Green Building

- Transportation

- Endowment Transparency

- Investment Priorities

- Shareholder Engagement

In its second year, the report card assesses and reports on 200 of the largest higher learning institutions in North America. Data collection took place from June through to September 2007. Based on 39 indicators, points were allocated to an individual school's policies and practices. A full letter grade of either A, B, C, D, E, or F (i.e. no plus or minus) was given for each of the above areas, then an overall (plus or minus) final grade, e.g. A+ or B-, was given (Sustainable Endowments Institute, 2007).

Information was gathered first from publicly available documentation. Three surveys were then sent to each school: 1) A Campus Survey covering the Administration, Climate Change and Energy, Green Building, and Transportation; (2) A Dining Services Survey covering the Food and Recycling category; and (3) An Endowment Survey covering the Endowment Transparency, Investment Priorities and Shareholders Engagement categories. 
The results have been positive for institutions that have been reviewed in both 2007 and 2008 report cards. These results show that:

- 68 percent of schools reviewed have improved their grade.

- More schools are taking pro-active steps to tackle climate change through aggressive carbon reduction commitments. This has increased from 14 to over 50 percent of schools.

- Green buildings policies are becoming the norm: a year ago only 48 percent of schools have green building policies, this has now risen to 69 percent.

- Endowment investment in renewable energy funds more than tripled amongst schools from 9 to 31 percent (Sustainable Endowments Institute, 2007).

Over recent years, a significant shift has occurred in the degree to which climate change is the subject of active institutional engagement. For example, the Sustainable Endowments Institute found in its review that the proportion of schools committing to reductions in GHG emissions tripled (from 14 to 50 percent) from 2006 to 2007, and more than 25 percent of schools have committed to achieving long term carbon neutrality by signing the ACUPCC (Sustainable Endowments Institute, 2007).

Both the ACUPCC and the CSRC prove that many of North American higher learning institutions are taking issues of climate change and campus sustainability seriously and have instituted proactive measures to reduce their environmental impact.

\subsubsection{Case Studies}

\section{The University of California, Santa Barbara}

The University of California, Santa Barbara is located on the California coast about 160 kilometres from Los Angeles. The campus size is 400 hectares. It has 20,000 student enrolments and a faculty size of just over 1000. In May 2006, the Bren School of Environmental Science and Management, (a department of the university), supported the completion of a Masters project entitled "Changing the Campus Climate: Strategies to Reduce Greenhouse Gas Emissions at the University of California, Santa Barbara" (Ahmend, et al, 2006). The group project was a requirement of all students enrolled in the Masters programme. 
The project was divided up into two main phases, analysis and implementation. In the analysis phase, an inventory was conducted of the main sources of GHG emissions on campus. Projected changes, mitigation strategies, development of criteria for selecting mitigation strategies and analysis of emission reduction targets were also inventoried. In the implementation phase, barriers to and opportunities for the university becoming carbon neutral were investigated. These two phases were conducted with the aim of prompting the University to reduce its emissions and receive the associated benefits involved.

In order to create a GHG inventory for UCSB, the inventory tool Clean Air-Cool Planet Campus Climate Action Toolkit (CCAT) was used. Although the inventory included a wide range of sources, including emissions from electricity consumption, vehicle fleet, commuting, air travel, refrigerator emissions and solid waste, the inventory only analysed GHG emissions required of their university by the California Climate Action Registry ${ }^{6}$. The GHG inventory analysed data over a 15-year period, dating from 1990 (Clean Air-Cool Planet, 2000).

By successfully certifying its GHG emissions inventory with the California Climate Action Registry, UCSB became the second UC campus to earn the distinction of Climate Action Leader. The California Climate Action Registry is a non-profit public/private partnership that encourages organisations to voluntarily register their GHG emissions. The registry, created by the California legislature in 2000, has over 75 major companies, cities, government agencies and NGOs registered. The intent of the registry is to encourage voluntary actions to increase energy efficiency and decrease GHG emissions (Levin, 2006).

The UCSB GHG inventory found that electricity is the single largest source of GHG emissions, corresponding to two-thirds of total emissions, followed by natural gas, corresponding to onethird of total emissions. It was found that the campus fleet's GHG emissions were negligible in comparison to the total GHG emissions.

To coincide with this project, a student guide was also released. This was a guide for university student groups planning to mitigate campus GHG emissions. Its purpose was to facilitate a student grassroots movement mobilising graduate students to lead the way to long-term climate

\footnotetext{
${ }^{6}$ The registry accepts GHG emission reports that include of all the Kyoto gases; however participants may opt to limit their reports to only $\mathrm{CO}_{2}$ during the first three years of participation. After the third year, participants are required to include all six of the Kyoto GHGs.
} 
solutions. Students at the university are also offered free public transport (Sustainable Endowments Institute, 2007: 161)

In March, 2007, the university signed the American College and University Presidents Climate Commitment. In an effort to attain carbon neutrality, the university aims to release its plan in late 2008, one year earlier than required by the ACUPCC agreement.

\section{College Sustainability Report Card Rating}

\begin{tabular}{|l|l|}
\hline Administration & A \\
\hline Climate Change and Energy & A \\
\hline Food and Recycling & A \\
\hline Green Building & A \\
\hline Transportation & A \\
\hline Endowment Transparency & B \\
\hline Investment Priorities & C \\
\hline Shareholder Engagement & D \\
\hline Overall Grade & B + \\
\hline
\end{tabular}

Figure 4: 2008 College Sustainability Report Card Rating for the University of California, Santa Barbara (Source: Sustainable Endowments Institute, 2007)

The University of California received a higher grade (Figure 4) in 2008 College Sustainability Report Card than the B grade received in the 2007 edition.

\section{Oberlin College, Ohio}

Oberlin College is a private liberal arts college, located in Ohio, with a student population of less than 3000 (Oberlin, 2006). Located on-site is the Adam Joseph Lewis Center for Environmental Studies (AJLC), an environmentally benign college building that has won several awards over the last few years (Orr, 2002: 162). The building includes photovoltaic (PV) panels to provide for the building's energy needs, with some of this energy being sold back to the local power grid; active and passive heating systems, including geothermal wells and large south-facing windows to control the indoor climate; an integrated building-landscape system to stimulate local flora and fauna; a Living Machine that is ecologically engineered, which combines elements of conventional wastewater technology with the purification process of a wetland ecosystem to treat 
and recycle the building's wastewater; a weather station to monitor the local climate; and building materials that were selected for their low energy consumption and low levels of toxicity.

The college has indicated its intention to become carbon neutral by 2020, a project established by Professor David Orr, Chair of Oberlin's Environmental Studies Department. The project's objective is to demonstrate leadership within its community by reducing GHG emissions produced by the college - ideally becoming carbon neutral - at the lowest possible costs or, if possible, at a profit. The latter scenario could be achieved by selling excess energy produced at the college back to the grid (Heede and Swisher, 2002).

Background work for this decision was provided by The Rocky Mountain Institute, which prepared a 125-page document for Oberlin College outlining a proposal that would make the college completely carbon neutral by the year 2020. The core objectives of the Oberlin 2020 project are the following (Heede and Swisher, 2002: 74):

- To assess the technical, economic, and GHG reduction feasibility of achieving "climate neutrality" for the college by the year 2020 .

- To identify the best combination of smart design, new cost-saving technology, campuswide policy initiatives, innovative financing mechanisms and a carefully phased 20-year (2001 - 2020) implementation plan to achieve the climate neutrality aspiration.

- To motivate the Oberlin College Trustees to support the project's approval, financing and implementation.

- To create, publish and promote a model GHG reduction strategy and implementation plan for other like-minded campuses.

The report presented four scenarios, with Oberlin's plan most consistent with the third:

1. The "baseline" 'BAU' scenario involves the university buying offsets. This scenario would require no special effort to save energy or reduce emissions to achieve neutrality.

2. The "no-brainer" (low-hanging fruit) scenario includes low cost-efficiency measures that rely on conservation technologies and modest levels of investment, resulting in the fastest paybacks and highest rates of return. 
3. The "no-regrets" (aggressive energy and co-generation) scenario includes aggressive but cost-effective energy efficiency and co-generation measures. This scenario requires higher levels of investment and slower returns.

4. The "no-prisoners" (carbon neutral) scenario is more aggressive and reduces carbon emissions to zero, and involves no purchasing of carbon offsets. It relies on higher levels of capital investment.

The report included three main strategies for cutting carbon emissions: on-site reductions, carbon sequestering and carbon offset purchasing. The report also outlined implementation and financing mechanisms. These included a GHG inventory, assessment of potential options for GHG reductions, barriers and risk assessment, and policy and financing options for all four scenarios.

By investing in co-generation measures, Oberlin College may be able to sell excess electricity back to the Ohio electricity grid and, in doing so, reduce emissions produced from the grid and then earn carbon offset credit. This is due to the fact that natural gas co-generation produces far fewer carbon emissions than the conventional power grid in Ohio. Oberlin College could achieve carbon neutrality without having to buy any external offsets (Heede and Swisher, 2002: 86).

In November 2006, Oberlin College became one of the first four higher learning institutes in the USA to sign the American College and University Presidents Climate Commitment (ACUPCC).

\section{College Sustainability Report Card Rating}

\begin{tabular}{|l|l|}
\hline Administration & A \\
\hline Climate Change and Energy & A \\
\hline Food and Recycling & A \\
\hline Green Building & A \\
\hline Transportation & C \\
\hline Endowment Transparency & D \\
\hline Investment Priorities & A \\
\hline Shareholder Engagement & A \\
\hline Overall Grade & B- \\
\hline
\end{tabular}

Figure 5: 2008 College Sustainability Report Card Rating for Oberlin College (Source: Sustainable Endowments Institute, 2007) 
Oberlin College received a higher grade (Figure 5) in 2008 College Sustainability Report Card than $\mathrm{C}+$ grade received in the 2007 edition.

\section{Harvard University - Cambridge, Massachusetts}

Harvard University is a large urban university located in Cambridge, Massachusetts, with more than 18,000 degree candidates enrolled, including undergraduates and students in 10 graduate and professional schools, 13,000 students within the Harvard Extension School, and more then 2,000 faculty staff and 14,000 general staff members.

The Harvard Green Campus Initiative is a wide-ranging campus sustainability programme. It employs a staff of 16 full-time professionals and 40 part-time student employees. The initiative involves sustainable behavioural change programmes with results that have saved the university considerable amounts of money through energy savings in residential dorms and faculty laboratories, renewable energy projects, performance building services, on-site solar panels, geothermal projects, bio-diesel for the university's vehicle fleet, a waste programme with a recycling rate of over 45 percent, a green campus loan fund and provision for a GHG inventory.

Harvard uses the Clean Air - Cool Planet Campus Carbon Calculator to assess GHG emissions. Though the university started its GHG inventory in 2001, as of yet, there are no specific reduction goals or targets for GHG reductions as the university is still assessing various GHG reduction scenarios for cost-effectiveness. Another barrier to Harvard's commitment to a specific plan is the decentralised nature of the University: with every school possessing independent decisionmaking power, consensus among all schools must be reached before a GHG commitment can succeed (Martin, 2008).

Approximately 7 percent of the university's total energy is offset by the purchase of renewable energy credits, making Harvard one of the largest university green energy purchasers in the USA. More than US\$12 million of the Green Campus Loan Fund ${ }^{7}$ has been invested in 110 projects. According to the Green Campus Initiative web-site, projects average a payback period of only five years and a return on investment of 33 percent. Environmental savings include over 30

\footnotetext{
7 The Green Campus Loan Fund provides capital for high performance campus design, operations, maintenance, and occupant behaviour projects. The Harvard Green Campus Initiative promotes and administers the fund.
} 
million $\mathrm{kg}$ of $\mathrm{CO}_{2}$ emission reductions, 48 million litres of water, and 100,000 $\mathrm{kg}$ of solid waste per year (pers. comm. Martin, 2008).

\section{College Sustainability Report Card Rating}

\begin{tabular}{|l|c|}
\hline Administration & A \\
\hline Climate Change and Energy & A \\
\hline Food and Recycling & A \\
\hline Green Building & A \\
\hline Transportation & B \\
\hline Endowment Transparency & D \\
\hline Investment Priorities & C \\
\hline Shareholder Engagement & A \\
\hline Overall Grade & A- \\
\hline
\end{tabular}

Figure 6: 2008 College Sustainability Report Card Rating for Harvard University- Cambridge, Massachusetts (Source: Sustainable Endowments Institute, 2007)

This grade rating (Figure 6) remains unchanged from the previous year's rating.

\section{Middlebury College}

Middlebury College is a liberal arts college in Vermont. There are over 2350 students who attend the college. The college attempts to incorporate an environmental ethic in all its undertakings (Dagan, 2002). An Environmental Council was formed comprising students, staff and faculty. The role of the council was to advise the Campus President on campus environmental issues. A main focus of the council was on the college's emissions of GHG emissions, with the aim of net reductions. In 2002 the college conducted an extensive emissions inventory, adapting the toolkit developed by Clean Air - Cool Planet. The inventory was compiled for the years 1990-2000.

In 2003, Middlebury College prepared a report on reducing the college's impact on the climate. The report, entitled 'Carbon Neutrality at Middlebury College: A Compilation of Potential Objectives and Strategies to Minimise Campus Climate Impact', involved the work of 16 students from the 2003 'Scientific and Institutional Challenges of Becoming Carbon Neutral' environmental studies class (Isham, 2003). The report aimed to present strategies that were 
economically feasible, provided the greatest net reduction of GHG emissions, or had the greatest long-term potential for significant mitigation of GHG emissions.

Both this report and the emissions inventory report were used by the college's Carbon Reduction Initiative Committee to develop a Climate Change Action Plan. In September 2006, the college announced that it would invest in a biomass plant, with an annual emission reduction of almost 12,500 metric tons. The estimated cost for this project is expected to be US\$11 million, and will be funded through loans and a state grant.

In January 2007, a proposal was developed to eliminate the college's net carbon emissions by 2016. The proposal outlined the educational opportunities of carbon neutrality for the college and detailed several infrastructural changes needed to reduce the college's carbon footprint. These projects included heating and cooling, electricity, transportation, waste and purchasing, and architecture and planning. In May 2007, the Middlebury College board of trustees approved a plan to become a carbon neutral institution by 2016 (Kloman, 2007). In the following month, the college signed the ACUPCC.

\section{College Sustainability Report Card Rating}

\begin{tabular}{|l|c|}
\hline Administration & A \\
\hline Climate Change and Energy & A \\
\hline Food and Recycling & A \\
\hline Green Building & A \\
\hline Transportation & A \\
\hline Endowment Transparency & C \\
\hline Investment Priorities & A \\
\hline Shareholder Engagement & A \\
\hline Overall Grade & A- \\
\hline
\end{tabular}

Figure 7: 2008 College Sustainability Report Card Rating for Middlebury College (Source: Sustainable Endowments Institute, 2007)

Middlebury College received a higher grade (Figure 7) in the 2008 CSRC rating than the B+ grade received in the 2007 edition. 


\section{Lewis and Clark College}

Lewis and Clark College is a small liberal arts college in Portland, Oregon, with fewer than 3000 students. In 2001, the college estimated its emissions for the years 1990 and 2000. This audit was conducted because the college, although not required to, wanted to become Kyoto compliant. This meant setting a target to reduce its emissions by 7 percent during the Kyoto period. Over and above this requirement, however, the college decided it would reduce its 1990 GHG emissions by 10 percent. The college became the first higher education institute in the USA to become Kyoto compliant (Dautremont-Smith, 2003).

The college believed that there existed little difference between buying offsets and investing in energy efficacy upgrades, as both require a financial investment. The economic argument put forth was that the cost of making further emissions reduction investments on-campus would be greater than the cost of purchasing an equivalent amount of offsets. Short-term on-campus emission reduction initiatives were viewed as too costly, more resource intensive and produced fewer external social benefits than offset projects.

These social benefits included investment in wind power projects, car pooling projects, forestry and a landfill gas collection power project. The university also intended to implement on-campus emission reductions over the long term with the goal of achieving carbon neutrality by the earliest possible date (Dautremont-Smith, 2003). In 2006, Lewis and Clark signed the ACPUCC and made a commitment to become carbon neutral (Sustainable Endowments Institute, 2007). Lewis and Clark College has not been evaluated by the college sustainability report card rating.

\subsection{United Kingdom}

As in the previous section covering the USA, the initiatives intended to facilitate the institutional development of GHG reductions programmes in the UK are outlined in this section. This is followed by four case studies examining UK tertiary institutions' response to such initiatives. Although several universities in the UK have committed to GHG reduction initiatives, at the time of writing, no universities had committed to any carbon neutral target. The last sub-section presents an example of a university as an agent of change within its local community. 
The UK's government has set a target of 60 percent $\mathrm{CO}_{2}$ emissions reduction by 2050 . The UK's higher learning sector will need to reduce emissions by 2 percent per year to meet this target (Fawcett, 2005). The Carbon Trust is one organisation that aims to help UK organisations to reduce their emissions.

\subsubsection{The Carbon Trust}

The Carbon Trust is a UK-wide private company set up by the Government in response to the country's increasing GHG emissions. Its focus is to move the UK towards a low-carbon economy. The Trust works with UK businesses and the public sector to create business-oriented solutions in five key areas: insights, innovation, enterprise, investments and solutions. By developing these five key areas, the Trust aims to develop and finance low-carbon enterprise.

The Trust is funded by the Department for the Environment, Food and Rural Affairs; the Department for Business; Enterprise and Regulatory Reform; the Scottish Executive; the Welsh Assembly Government and; Invest Northern Ireland (The Carbon Trust, 2007).

The Trust has set up a Higher Education Carbon Management programme (HECM), which is designed specifically for universities and higher education colleges, providing them with support and guidance to reduce their emissions and energy costs. However, it has no specific carbon neutral objective. Once accepted into the programme, a university must have senior management support, form a team or committee and follow a five step process (Figure 8) to reduce emissions. The Trust recommends that the team leader dedicate at least two days a week for 10 months to the process. 
Step One: Mobilise the organisation

Building a team and determining the scope

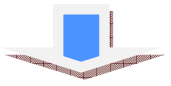

Step Two: Set baseline, forecast and targets

Setting the baseline for the programme and its goals

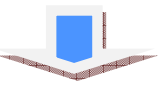

Step Three: Identify and quantify options

Identify the risks and opportunities presented by climate change

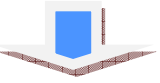

Step Four: Finalise Strategy and Implementation Plan

Design a cost-effective strategy to cut emissions and save money

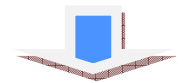

Step Five: Implement plan

Complete with budgets, targets, and success metrics

Figure 8: The HECM Five Step Process

As of 2007, the Carbon Trust's programme has so far involved 33 universities and identified potential savings of $£ 12$ million and 125,000 tonnes of $\mathrm{CO}_{2}$ for the organisations involved. Universities in the UK that are already committed to the Carbon Management programme include: Coventry University, King's College London, Leeds Metropolitan University, London Metropolitan University, Oxford Brookes University, The Open University, University of Birmingham, University of Bradford, University of Cambridge, University of Leeds, University of Southampton, University of St Andrews, University of Strathclyde, University of Sunderland, University of Sussex, University of Teesside, University of the West of England, University of Wales (Aberystwyth), University of Warwick, and the University of York (Carbon Trust, 2007).

\subsubsection{Case Studies}

\section{The University of Cambridge}

The University of Cambridge has an annual energy and water bill of over $£ 9$ million and an annual carbon footprint of 55,000 tonnes. In an effort to improve energy efficiency, the university 
signed up to the Carbon Trust Higher Education Carbon Management programme. The Trust helped the university identify several areas that needed improvement. These included:

- Improving the Building Energy Management Systems and thus making improvements in the heating and cooling of buildings.

- Installing more efficient lighting system.

- Improvements in the monitoring of energy usage in individual buildings.

- An improvement in the insulation of the university's piping system.

A plan was developed to reduce the university's GHG emissions by 10 percent from 2006 to 2010, despite projected expansion and growing demand for energy (University of Cambridge, 2007). By following the Trust's recommendations, the university expects to reduce carbon emissions by 16,000 tonnes and save $£ 6$ million in energy costs. The Trust helped the university secure finance worth over $£ 300,000$ to implement these improvements (The Carbon Trust, 2007).

\section{The University College London}

The University College London (UCL) is England's third oldest university. It has a community of more than 24,500 staff and students, occupying 120 sites. The total energy bill for the university is more then $£ 4$ million a year. Working with the Carbon Trust, immediate savings of $£ 119,000$ were identified for the three largest sites by following simple technical improvements and staff/student energy awareness programmes. It is estimated that if the Trust's recommendations are followed at all of UCL's 120 sites, savings of up to $£ 750,000$ a year could be achieved (The Carbon Trust, 2006).

In late 2007 the university conducted a carbon audit and is currently working with the Carbon Trust on an environmental strategy aiming to reduce GHG emissions by 20 percent from 2008 to 2012. This five-year plan will be submitted for audit to the Carbon Trust in early 2008 and will then be submitted to the UCL Council before implementation (Anderson, 2008).

\section{University of St Andrews}

The University of St Andrews is Scotland's oldest university and the UK's third oldest. In the academic year 2006/07 there were around 6700 students and 1500 staff members. 
Through the Carbon Trust, St Andrews was the first Scottish university to sign up to the Higher Education Carbon Management programme. The adopted programme constitutes one objective under the university's Sustainable Development Strategy. This aims to reduce energy consumption and waste, and promote the use of renewable energy.

The university has secured $£ 1$ million over a two-year period for investment in energy efficiency programmes. Although there is no timeframe or targets set, the university aims to become the UK's first certified carbon neutral university (pers. comm. Yarr, 2007).

\subsubsection{An Example of a University as an Agent of Change}

\section{The University of Chester}

The University of Chester made a five-year commitment, beginning 2006, to supporting Aston Hayes Village in its efforts to become carbon neutral. If successful, it will be England's first carbon neutral village (Alexander, et al, 2007). Working closely with the village, the university has provided expertise to carry out a baseline GHG emissions survey and a group of students to monitor the village's success in reducing and mitigating emissions. While this project is a community led initiative, it demonstrates the leadership role universities can play. Being inspired by this project, the Chester City Council decided to invest in the CRed package, a web-based system. This system allows individuals to monitor their own emissions. The university is working closely with the council, by providing advice, analysis and reporting services.

\subsection{Summary}

Numerous universities in the USA and UK are beginning to record and reduce their GHG emissions. Carbon neutrality is a viable target to which college and university presidents and chancellors in North America are committing. This is clear given the numbers signing up to the ACUPCC. By agreeing to the commitment, higher learning institutes lead by example. This is a highly visible example witnessed by government, industry and the public, signifying academies' visibility in the community and their role as centers for innovation, emphasising the fact that they have a critical role in mitigating climate change. 
One factor that all these universities have in common is that they are not going through the process of emission reductions by themselves. Support, advice and know-how are available, designed specifically for universities seeking to become carbon neutral and reduce emissions. Universities in North America can use the carbon-auditing tool, Clean Air- Cool Planet, and make a pledge of carbon neutrality through the ACUPCC. Universities in the UK can reduce their emissions with the advice and financial help of the Carbon Trust. Higher learning institutes in both regions are also able to learn from each other by establishing networks.

Other significant features include the integration of carbon neutrality into academic courses, as a teaching resource for programmes such as environmental studies or energy management, and into wider scope sustainability initiatives. Carbon reduction initiatives can also save money through energy efficiency initiatives, although this often requires initial capital investment. 


\section{The Carbon Footprint for VUW - Carbon Auditing}

This chapter will focus on objective two of the research, reviewing the aspects of the carbon audit of VUW conducted by URS, and compare the results with an estimation obtained by using another auditing tool from the New Zealand Business Council for Sustainable Development. The subsequent section will present the costing to offset the 2006 VUW GHG footprint as estimated by URS, as evaluated by carboNZero. Following this, the 2007 VUW GHG footprint will be presented. Finally, an analysis of the results will be carried out.

\subsection{Results: Identifying a 2006 GHG footprint for VUW}

\subsubsection{Identifying a GHG footprint for 2006 as estimated by URS}

URS Ltd is a professional services company that provides organisations with engineering and environmental advice across New Zealand, Asia Pacific and elsewhere. In 2007, an environmental audit for 2006 was conducted by URS for VUW. Following this audit, a report was prepared by URS that involved a high-level environmental review and inventory of VUW's environmental performance data that included GHG emissions. The GHG Protocol Initiative was used as the basis for the carbon audit process by URS (URS, 2007).

\section{Selection of emission factors}

URS had investigated a variety of different sources to obtain the emission factors that were used in this project. These sources included the Ministry for the Environment (MfE), the New Zealand Business Council for Sustainable Development (NZBCSD), the Electricity Efficiency and Conservation Authority (EECA), the Ministry of Economic Development (MED), the carboNZero Scheme of LandCare Research and the Electricity Commission (URS, 2007). 
Table 1: URS Emission Factors applied in the VUW emissions audit (URS, 2007)*

\begin{tabular}{|c|c|c|c|}
\hline Emission Source & Units & $\begin{array}{l}\text { Emission Factor } \\
\left(\mathrm{kg}^{8}\right.\end{array}$ & Source \\
\hline \multicolumn{4}{|c|}{ Scope I } \\
\hline \multirow{2}{*}{ Petrol Fleet } & Litres & 2.32 & \multirow{2}{*}{ IRD } \\
\hline & $\mathrm{km}$ travelled & 0.2 & \\
\hline Diesel Fleet & Litres & 2.71 & IRD \\
\hline Gas & kwh & 0.188 & NZBCSD \\
\hline \multicolumn{4}{|c|}{ Scope II } \\
\hline Electricity & kwh & 0.625 & $\mathrm{EC}$ \\
\hline \multicolumn{4}{|c|}{ Scope III } \\
\hline $\begin{array}{l}\text { Taxis, rental cars, } \\
\text { mileage }\end{array}$ & Litres & 2.32 & IRD \\
\hline Domestic air travel & $\mathrm{km}$ travelled & 0.18 & NZBCSD \\
\hline $\begin{array}{l}\text { International air } \\
\text { travel }\end{array}$ & $\mathrm{km}$ travelled & 0.11 & NZBCSD \\
\hline Car & $\mathrm{km}$ travelled & 0.2 & CarboNZero \\
\hline Motorcycle & km travelled & 0.08 & CarboNZero \\
\hline Bus & $\mathrm{km}$ travelled & 0.09 & CarboNZero \\
\hline Train & $\mathrm{km}$ travelled & 0.15 & CarboNZero \\
\hline Waste & $\mathrm{kg}$ & 0.45 & CarboNZero \\
\hline Virgin Paper & $\mathrm{kg}$ & 1.63 & Norske Skog \\
\hline $\begin{array}{l}\text { Paper with less } \\
\text { than } 50 \text { percent } \\
\text { recycled content }\end{array}$ & $\mathrm{kg}$ & 0.815 & Norske Skog \\
\hline $\begin{array}{l}\text { Paper with more } \\
\text { than } 80 \text { percent } \\
\text { recycled content }\end{array}$ & $\mathrm{kg}$ & 0.163 & Norske Skog \\
\hline
\end{tabular}

${ }^{8}$ IRD (Implementing the Carbon Tax 2005 Consultation Paper, NZBCSD (GHG Protocol of New Zealand Business Council for Sustainable Development), EC (Electricity Emission Factor Review, 2004), CarboNZero (Landcare Research CarboNZero Household calculator), Norske Skog (Norske Skog New Zealand Sustainability Report, 2005)

While emission factors and data quality are considered to be as accurate as possible for Scope 1 and 2 emissions, Scope 3 emissions have been either derived or estimated (URS, 2007). 
Table 2: VUW 2006 Overall GHG profile estimate by emission source (URS NZ)

\begin{tabular}{|l|l|l|}
\hline Type of Emission & Source & Tonnes $\mathrm{CO}_{2} \mathrm{e}$ \\
\hline \multirow{4}{*}{ Business Travel } & Taxi & 25.97 \\
\cline { 2 - 3 } & Rental cars & 23.20 \\
\cline { 2 - 3 } & Mileage & 7.18 \\
\cline { 2 - 3 } & University Fleet & 129.22 \\
\cline { 2 - 3 } & Air Travel & 3047.84 \\
\hline Total Business Travel & & 3233.40 \\
\hline \multirow{2}{*}{ Commuting } & Students & 3830.68 \\
\cline { 2 - 3 } & Staff & 1291.92 \\
\hline Total transport & & 8356.00 \\
\hline Energy & Electricity & $12,235.80$ \\
\cline { 2 - 3 } & Gas & 3328.53 \\
\cline { 2 - 3 } & Diesel generation & 1.20 \\
\hline Total (excl. elect.)* & & $15,565.53$ \\
\hline Total Energy (incl. elect.) & & 3329.73 \\
\hline Total Energy (excl. elect.) & & 292.52 \\
\hline Waste & & 456.15 \\
\hline Paper & & $24,670.20$ \\
\hline Total (incl. elect.) & & 12.40 \\
\hline \multirow{2}{*}{ Totre } & & \\
\hline
\end{tabular}

* Electricity is currently purchased from Meridian Energy, a certified carbon neutral supplier

The results from URS show that the carbon footprint for VUW in 2006 was around 25,000 tonnes of $\mathrm{CO}_{2}$ e. Excluding electricity consumption, due to it being purchased from Meridian, a carbon neutral energy supplier (carboNZero, 2007a), the GHG footprint for 2006 was around 12,400 tonnes. 
Figure 9: 2006 Total GHG Emissions of VUW, as estimated by URS (URS, 2007)

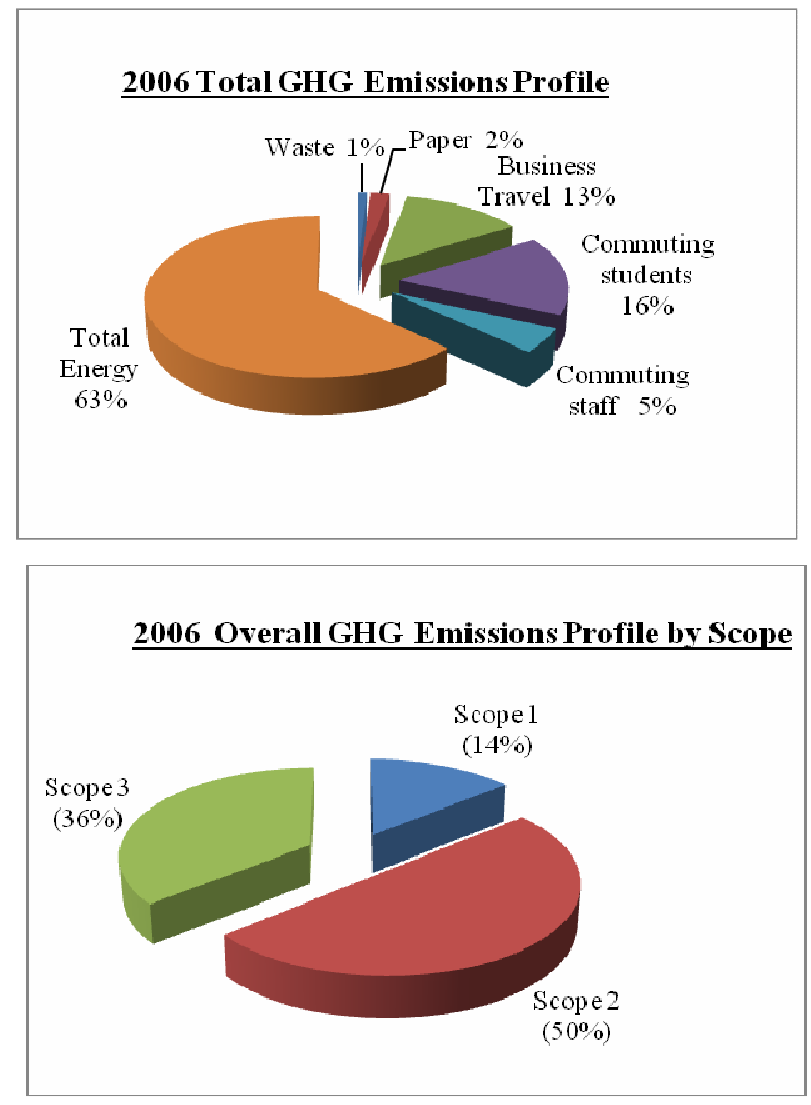

Figure 10: 2006 GHG Emissions Profile of VUW by Scope, as estimated by URS (URS, 2007)

\subsubsection{Identifying a 2006 GHG Emissions for VUW using the NZBCSD assessment tool}

As a comparison to the GHG footprint for VUW as estimated by URS, the New Zealand Business Council for Sustainable Development's GHG Emissions web-based assessment tool was also used. The emission factors listed on the NZBCSD website are based on 2002 emissions factors. Emissions from several sources have not been included, due to the NZBCSD web-based calculator tool not accounting for them. 
Table 3: NZBCSD Emission Factors (Source NZBCSD, 2002)

\begin{tabular}{|l|l|l|}
\hline Emission Source & Units & $\begin{array}{c}\text { Emission Factor } \\
\mathrm{kg}\end{array}$ \\
\hline \multirow{2}{*}{ Petrol Fleet } & Litres & 2.32 \\
\cline { 2 - 3 } & $\mathrm{km}$ travelled & 0.244 \\
\hline Diesel Fleet & Litres & 2.71 \\
\hline Gas & Kwh & 0.188 \\
\hline Electricity & Kwh & 0.230 \\
\hline Taxis, rental cars, mileage & Litres & 2.32 \\
\hline Domestic air travel & $\mathrm{km}$ travelled (medium haul) & 0.180 \\
\hline International air travel & $\mathrm{km}$ travelled (long haul) & 0.110 \\
\hline
\end{tabular}

Table 4: 2006 GHG Profile for VUW by Emissions Source, estimated using the NZBCSD tool

\begin{tabular}{|l|l|l|}
\hline Type of Emission & Source & Tonnes $\mathrm{CO}_{2} \mathrm{e}$ \\
\hline \multirow{4}{*}{ Business Travel } & Taxi & 25.97 \\
\cline { 2 - 3 } & Rental cars & 23.20 \\
\cline { 2 - 3 } & Mileage & 7.18 \\
\cline { 2 - 3 } & University Fleet & 129.22 \\
\cline { 2 - 3 } & Air Travel & 3047.84 \\
\hline Total Business Travel & & 3233.41 \\
\hline \multirow{2}{*}{ Commuting } & Students & 582.92 \\
\cline { 2 - 3 } & Staff & 849.85 \\
\hline Total transport & & 4666.18 \\
\hline Energy & Electricity & 4502.77 \\
\cline { 2 - 3 } & Gas & 3328.53 \\
\cline { 2 - 3 } & Diesel generation & 1.20 \\
\hline Total Energy (incl. elect.) & & 7832.5 \\
\hline Total Energy (excl. elect.) & & 3329.73 \\
\hline Total (incl. elect.) & & $12,498.68$ \\
\hline Total (excl. elect.) & & 7995.91 \\
\hline
\end{tabular}


The estimate based on the NZBCSD's tool show that the carbon footprint for Victoria University in 2006 was 12,500 tonnes of $\mathrm{CO}_{2} \mathrm{e}$ (Table 4). Reconciliation between the VUW carbon footprint estimate by URS and the carbon footprint estimated for VUW, using the NZBCSD tool, will be explained in below in Section 5.4. The 2006 Total GHG Emissions of VUW estimated using the NZBCSD tool is show in Figure 11. The 2006 GHG Emissions of VUW by source, estimated using the NZBCSD tool is show in Figure 12.

Figure 11: 2006 Total GHG Emissions of VUW estimated using the NZBCSD tool

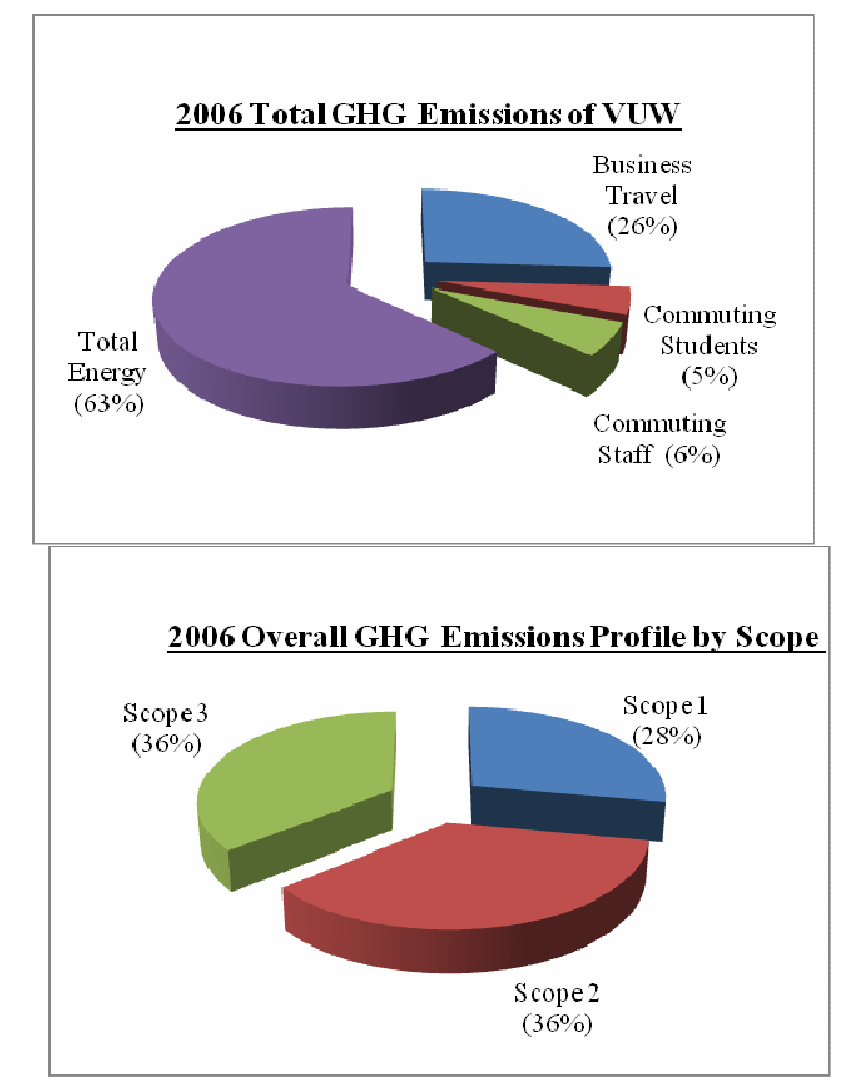

Figure 12: 2006 GHG Emissions of VUW by source, estimated using the NZBCSD tool

\subsection{CarboNZero costing for 2006 GHG footprint}

CarboNZero is a carbon emissions management and certification programme developed by Landcare Research. The programme is based on research carried out over the last decade and has been funded by the Foundation for Research, Science and Technology. It provides the necessary tools to assist organisations and individuals to estimate and reduce their carbon footprint.

CarboNZero has worked with organisations such as the New Zealand Wine Company (owners of the Grove Mill brand), Toyota NZ and South Pacific Pictures (Renowden, 2007). There are three key steps involved. 
1. Measuring the carbon footprint

2. Managing this footprint and reducing it where possible

3. Mitigating the carbon footprint where emission reductions are not possible.

The offsets used by carboNZero are Kyoto-consistent carbon credits from verified schemes such as renewable energy generation and the regeneration of NZ native forests (EBEX21 carbon credits), where landowners agree to allow marginally productive land to regenerate as native bush and are paid for the carbon the new growth takes out of the atmosphere.

\subsubsection{Financial cost for VUW to become carbon neutral}

Based on the URS audit, VUW can become carbon neutral through the carboNZero programme at an approximate cost of $\$ 330,000$ (GST exclusive) for the first year, as demonstrated in the breakdown below (Table 5):

Table 5: The carboNZero certification process cost breakdown (carboNZero, 2007b)

\begin{tabular}{|l|l|}
\hline carboNZero Step & Estimated Cost \\
\hline Measure & $\$ 25,000+$ GST \\
\hline Manage & No cost, unless consultancy required \\
\hline External Audit & No cost, URS Ltd Audit \\
\hline Mitigate & $\$ 254,200+$ GST \\
\hline Certification & $\$ 50,000+$ GST \\
\hline Total estimated cost: & $\$ 329,200+$ GST \\
\hline
\end{tabular}

\subsection{Results: Identifying a GHG footprint for 2007}

An internal VUW estimation for the 2007 VUW GHG footprint is shown below. This was calculated by Andrew Wilks, the Environmental Manager from Facilities Management. The emission factors used were based on the emission factors used by URS, except for the emission factor used for electricity. This was based on the Ministry for the Environment's 2007 average emission factor, which was 0.209 (tonnes $\mathrm{CO}_{2}$ ). 
Table 6: 2007 VUW Overall profile by emission source (pers. comm. Wilks, 2008)

\begin{tabular}{|l|l|l|}
\hline Type of Emission & Source & Tonnes $\mathrm{CO}_{2} \mathrm{e}$ \\
\hline \multirow{4}{*}{ Business Travel } & Taxi & 25.97 \\
\cline { 2 - 3 } & Rental cars & 23.20 \\
\cline { 2 - 3 } & Mileage & 3.53 \\
\cline { 2 - 3 } & University Fleet & 133.63 \\
\cline { 2 - 3 } & Air Travel & 1720.03 \\
\hline Total Business Travel & & 1906.36 \\
\hline \multirow{2}{*}{ Commuting } & Students & 3830.68 \\
\cline { 2 - 3 } & Staff & 1291.92 \\
\hline Total transport & & 7028.96 \\
\hline Energy & Electricity & 4095.30 \\
\cline { 2 - 3 } & Gas & 3062.71 \\
\cline { 2 - 3 } & Diesel generation & 2.27 \\
\hline Total (excl. elect.)* & & 7160.28 \\
\hline Total Energy (incl. elect.) & & 3064.98 \\
\hline Total Energy (excl. elect.) & & 282.53 \\
\hline Waste & & 280.84 \\
\hline \multirow{2}{*}{ Paper } & & 14.752 .61 \\
\hline \multirow{2}{*}{ Total (incl. elect.) } & & \\
\hline & & \\
\hline
\end{tabular}

Figure 13: 2007 Total GHG Emissions of VUW as estimated by VUW (pers. comm. Wilks, 2008)

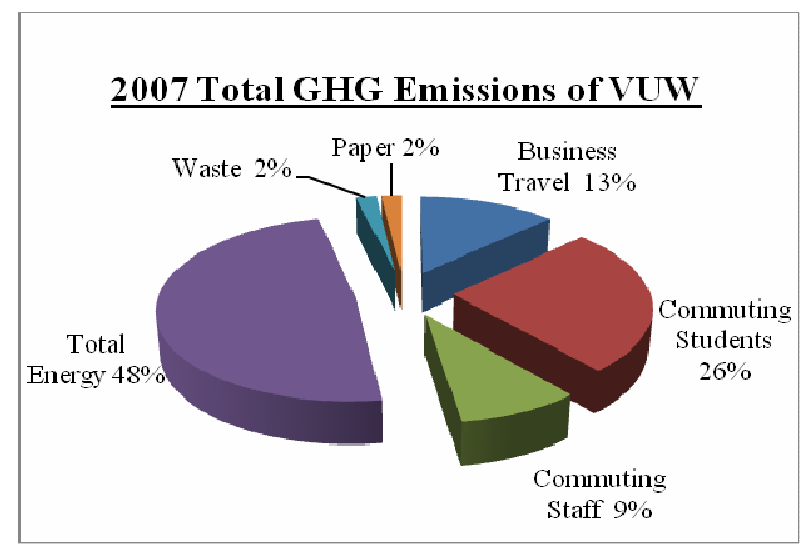




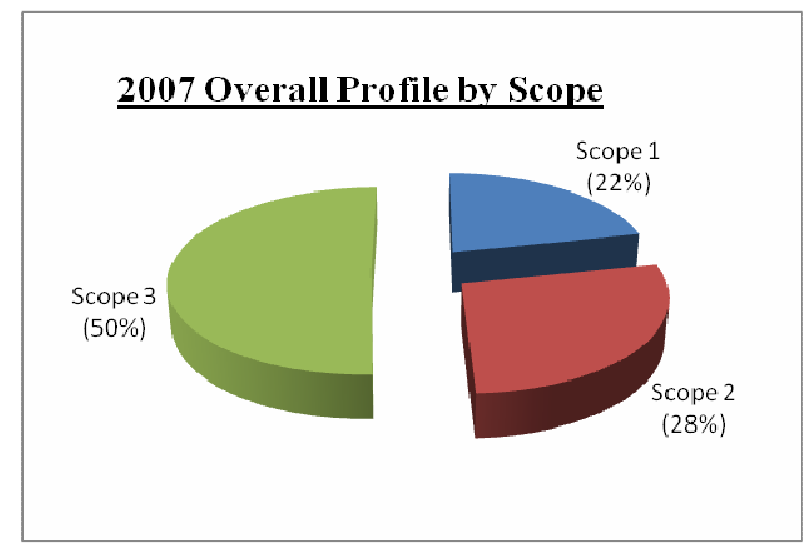

Figure 14: 2007 Overall Profile by Scope as estimated by VUW (pers. comm. Wilks, 2008)

\subsection{Analysis of VUW carbon audits}

\subsubsection{Comparing VUW's $2006 \mathrm{CO}_{2} \mathrm{e}$ profile estimation by URS and the NZBCSD calculator tool}

Comparing VUW's $2006 \mathrm{CO}_{2}$ e profile estimation by URS $(24,500)$ and the estimation using the NZBCSD tool $(12,500)$ gives a difference of 12,000 tonnes, almost 100 percent difference. At first glance, this difference is large; however, further analysis shows that a key difference between the two estimations are the emission factors used. For example, URS uses the marginal emissions factor of $0.625 \mathrm{~kg} \mathrm{CO}_{2} / \mathrm{kWh}$ for electricity, where the NZBCSD tool uses the average emission factor of $0.23 \mathrm{~kg} \mathrm{CO}_{2} / \mathrm{kWh}$.

The emission factor of 0.625 for electricity is obtained from the Concept Consulting Group and the MfE (CC Group, 2004). The emission factor for electricity of 0.23 is an average emission factor for electricity consumption in 2006, obtained from a Ministry for Economic Development report entitled 'New Zealand Energy Greenhouse Gas Emissions 1990 - 2006 (MED, 2007).

The URS estimate of emissions for electricity is 12,236 tonnes of $\mathrm{CO}_{2} \mathrm{e}$. This figure is obtained from:

$$
\begin{aligned}
\text { Emissions } & =\text { Total electricity used } * \text { emission factor } \\
& =19,577,279.18 \mathrm{kWh} * 0.625 \mathrm{kgCO}_{2} / \mathrm{kWh} \\
& =12,235,799.45 \mathrm{~kg} \mathrm{CO}_{2} \\
& =12,236 \text { tonnes } \mathrm{CO}_{2}
\end{aligned}
$$


However, if the average emission factor were used, it would give:

$$
\begin{aligned}
\text { Emissions } & =\text { Total electricity used } * \text { emission factor } \\
& =19,577,279.18 \mathrm{kWh} * 0.23 \mathrm{kgCO}_{2} / \mathrm{kWh} \\
& =4,502,774.11 \mathrm{~kg} \mathrm{CO}_{2} \\
& =4503 \text { tonnes } \mathrm{CO}_{2}
\end{aligned}
$$

This figure of 4503 tonnes of $\mathrm{CO}_{2}$ emission for electricity now approximates to the estimate obtained by using the NZBCSD tool. Using this figure instead of 12,236 tonnes $\mathrm{CO}_{2}$, gives a total overall profile of GHG emissions for VUW in 2006 of 16,937 tonnes $\mathrm{CO}_{2} \mathrm{e}$.

Another difference in emission factors is that of petrol, (kilometres travelled). URS uses an emission factor for petrol kilometres travelled of $0.2 \mathrm{~kg} \mathrm{CO}_{2} / \mathrm{km}$, whereas, the NZBCSD tool uses an emission factor of $0.244 \mathrm{~kg} \mathrm{CO}_{2} / \mathrm{km}$. URS obtained this emission factor from carboNZero, due to due to wanting to have consistency. 'It is a good practice to use factors from limited sources as they all have different methodology and assumptions behind them' (KarlikNeale, 2008).

Table 7: Comparison between commuting emission factors between URS and NZBCSD (URS, 2007)

\begin{tabular}{|l|l|l|l|}
\hline \multicolumn{1}{|c|}{ Emission Source } & \multicolumn{1}{c|}{$\begin{array}{c}\mathrm{km} \\
\text { travelled }\end{array}$} & Emission Factor $\mathrm{kg} \mathrm{CO}_{2} / \mathrm{km}$ & \multicolumn{1}{c|}{$\begin{array}{c}\text { Tonne } \\
\mathrm{CO}_{2}\end{array}$} \\
\hline $\begin{array}{l}\text { Commuting Students } \\
\text { Car (km travelled) }\end{array}$ & $2,389,000$ & 0.2 (from URS) & 448 \\
\cline { 3 - 4 } $\begin{array}{l}\text { Commuting Staff } \\
\text { Car (km travelled) }\end{array}$ & $3,483,000$ & 0.244 (from NZBCSD) & 583 \\
\cline { 3 - 4 } & & 0.244 & 697 \\
\hline
\end{tabular}

Another factor explaining the large difference is that the estimation obtained using the NZBCSD tool does not account for emissions produced by waste, paper usage, commuter travel by bus, train, or motorbike whereas URS does calculate for these emissions. Including emissions for waste produced by these sources (using emission factors from URS) increases the overall estimated profile using the NZBCSD tool by a total of 4697 tonnes, as shown in Table 8: 
Table 8: Estimated emissions from sources not included in the NZBCSD tool

\begin{tabular}{|l|l|}
\hline Emission Source & Tonnes $\mathrm{CO}_{2}$ \\
\hline Waste & 292.52 \\
\hline Paper usage & 456.15 \\
\hline Commuting Students & \\
\hline Bus & 548.10 \\
\hline Train & 2768.70 \\
\hline Motorcycle & 36.08 \\
\hline Commuting Staff & \\
\hline Bus & 98.46 \\
\hline Train & 488.70 \\
\hline Motorcycle & 8.16 \\
\hline Total & 4697 \\
\hline
\end{tabular}

When all these factors are taken into account, including the emission factor used by URS for commuting by car of $0.2 \mathrm{~kg} \mathrm{CO}_{2} / \mathrm{km}$, instead of the emission factor of $0.244 \mathrm{~kg} \mathrm{CO}_{2} / \mathrm{km}$, a new estimated emissions profile results: 
Table 9: Adjusted VUW GHG emissions profile for estimate obtained by the NZBCSD tool

\begin{tabular}{|c|c|c|}
\hline Type of Emission & Source & Tonnes $\mathrm{CO}_{2} \mathrm{e}$ \\
\hline \multirow{5}{*}{ Business Travel } & Taxi & 25.97 \\
\hline & Rental cars & 23.20 \\
\hline & Mileage & 7.18 \\
\hline & University Fleet & 129.22 \\
\hline & Air Travel & 3047.84 \\
\hline Total Business Travel & & 3233.41 \\
\hline \multirow{2}{*}{ Commuting } & Students & 3830.68 \\
\hline & Staff & 1291.92 \\
\hline Total transport & & 8356.00 \\
\hline \multirow[t]{3}{*}{ Energy } & Electricity & $12,235.80$ \\
\hline & Gas & 3328.53 \\
\hline & Diesel generation & 1.20 \\
\hline Total Energy (incl. elect.) & & $23,921.54$ \\
\hline Total Energy (excl. elect.) & & $11,685.75$ \\
\hline Waste & & 292.52 \\
\hline Paper Usage & & 456.15 \\
\hline Total (incl. elect.) & & $24,670.21$ \\
\hline Total (excl. elect.) & & 12,434 \\
\hline
\end{tabular}

This new GHG emissions estimate for VUW, obtained using the NZBCSD tool, is now equivalent to the GHG emissions estimate by URS.

\subsubsection{Criticism of the NZBCSD assessment tool}

The NZBCSD web-based assessment tool is limited in measuring the different scopes. It measures Scope I and Scope II emissions, but is limited in measuring Scope III emissions. The GHG Protocol Initiative suggests that an organisation should measure and account for Scope I and Scope II at a minimum. However, as illustrated by the two differing results, this excludes around 4,500 tonnes of GHG emissions between the estimation from URS and the estimation given by the NZBCSD tool. For a more complete estimation of the university's GHG footprint, emissions from Scope III should be included. 


\subsubsection{Further analysis of the 2006 GHG footprint for VUW}

As the 2006 GHG emissions footprint for VUW, as estimated by URS, is the official GHG footprint for the university, further analysis is given below. However, the GHG footprint is a rough estimate, due to the small amount of information available and the largely developmental stages of data recording at VUW. The data collected by URS had many limitations (URS, 2007; pers. comm. Wilks, 2007).

When excluding electricity consumption from the 2006 GHG footprint, a new GHG profile is given in Figure 15:

Figure 15: 2006 GHG Emissions Profile (excl. electricity) by source of VUW, as estimated by URS (URS, 2007)

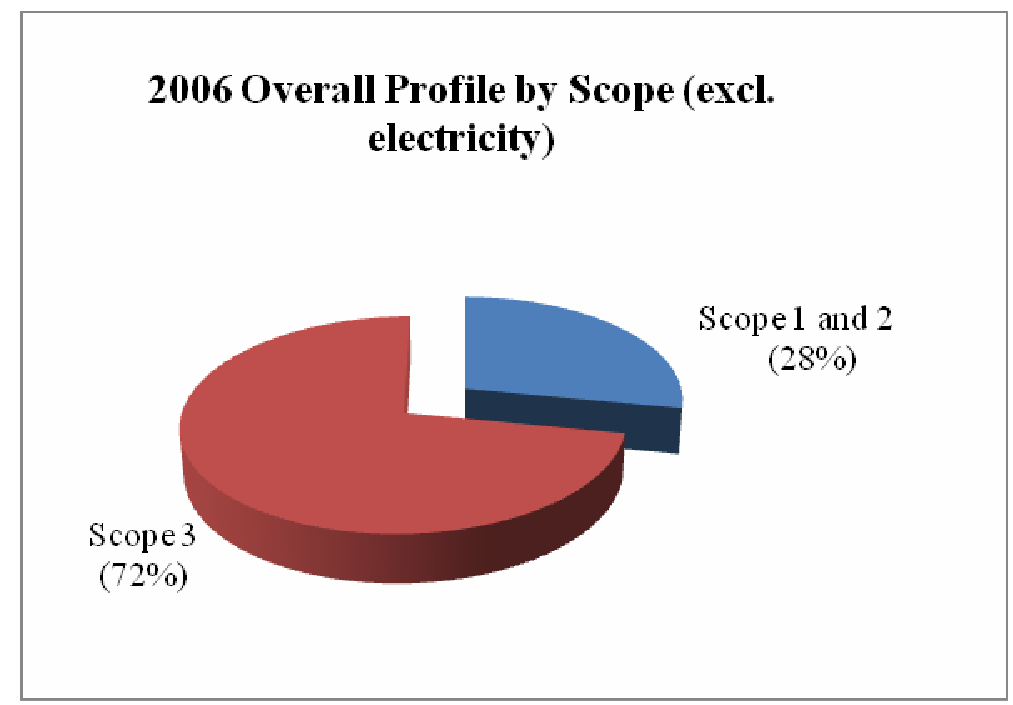

Scope III emissions, emissions that occur as a consequence of the university's activities, but are not owned or controlled by the company, account for the largest source of emissions produced by VUW's operations. Much of the data collected for Scope III sources have been estimated, due to lack of data availability (URS, 2007: 1-4). This situation is expected to improve over the next few years, as recording processes become more sophisticated, resulting in a more accurate VUW emissions footprint (pers. comm. Wilks, 2007). Scope I emissions are directly measured from the university's records (URS, 2007). 


\section{Energy}

The energy requirements at VUW are supplied by mains electricity and reticulated gas. The university has four back-up diesel generators, which are used for emergency lighting, IT servers, and for freezing (pers. comm. Wilks, 2007). The data collected for energy usage of the university was from direct measurement records. Emissions produced from energy are classified as Scope I (gas and diesel) and Scope II (electricity) emissions. Emissions from gas are a significant contributor to the university's GHG energy profile.

Figure 16: 2006 GHG Energy Emissions Profile of VUW, as estimated by URS (URS, 2007)

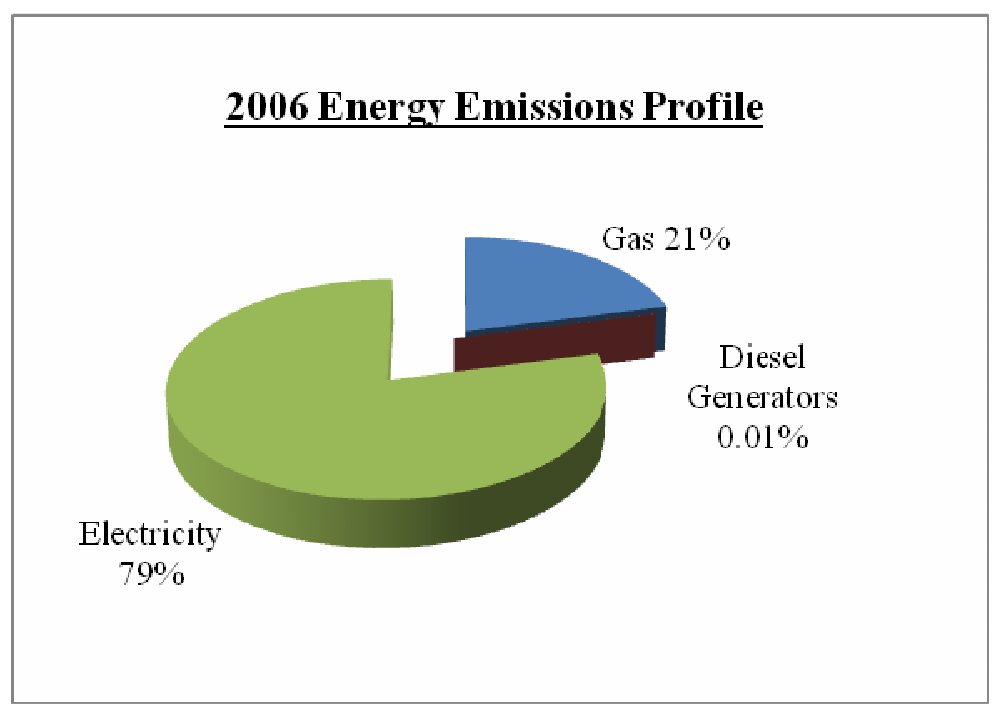

\section{Transport}

Leaving aside emissions produced from electricity from the overall emissions profile, the estimate GHG footprint provided by URS shows that transport is the largest contributor to the university's carbon footprint, representing 67 percent of the total GHG profile (12,434 tonnes) for 2006, comprising of: 
Table 10: 2006 GHG Travel Emissions Profile (excl. electricity) of VUW, as estimated by URS (URS, 2007)

\begin{tabular}{|l|l|}
\hline Source & $\begin{array}{l}\mathrm{CO}_{2} \text { Percentage } \\
\text { GHG footprint (excl. Electricity) }\end{array}$ \\
\hline Taxi & $0.21 \%$ \\
\hline Rental Cars & $0.19 \%$ \\
\hline University Fleet & $1.00 \%$ \\
\hline Private Mileage & $0.06 \%$ \\
\hline Air Travel & $24 \%$ \\
\hline Commuting Students & $31 \%$ \\
\hline Commuting Staff & $10 \%$ \\
\hline Total Transport & $100 \%$ \\
\hline
\end{tabular}

\section{1) Business Travel}

The data collected regarding the university's vehicle fleet is directly measured, whereas data collected from taxis, rental cars, mileage, and air travel are estimated. The university's fleet accounts for 4 percent of 2006 business travel emissions, which falls into Scope I emissions (within direct control of the university). 94 percent of emissions associated with business travel in 2006 are related to air travel. Air travel emissions are classes as Scope III emissions, as according to the GHG Protocol Initiative (see section 3.5.2), which are indirect GHG emissions that occur as a consequence of the university's activities (URS, 2007).

Air travel was also estimated on an assumed distance travelled per dollar spent, rather than on actual flight distance in kilometres (pers. comm. Wilks, 2008). Therefore, the accuracy of this estimation for 2006 is questionable.

\section{2) Commuting}

The data collated for commuting staff and students is based on the VUW travel survey conducted in 2007 (Opus, 2007). The survey sample size was 10 percent of all staff and 7 percent of all students. This was then extrapolated to represent all staff and students. Therefore it has to be acknowledged that this is a small sample size. Commuting students and staff represented $41 \%$ of the total carbon footprint for VUW (URS, 2007). Commuting by car is the most carbon intensive mode of travel by VUW staff. 
According to the 2007 VUW travel survey, $40 \%$ of VUW Staff commute by car, which accounts for 54\% of emissions generated by staff commuting related emissions (Opus, 2007). In regards to commuting by students, the largest proportion of emissions generation is related to travel by train. VUW cannot make improvements to this without the co-operation of Greater Wellington Regional Council, which controls the public transportation system in the region. VUW is in the process of implementing a university wide travel plan, but this has not yet been finalised.

\section{Waste}

Waste produced by VUW included in the URS audit was defined as 'any solid waste collected on campus for disposal, recycling or re-use' (URS, 2007). The university collects solid waste for disposal via skip bins. Gantry-style bins have their weights directly measured, while the solid waste data from regular skip bins is estimated from volume multiplied by the number of empty skip bins (pers. comm. Wilks, 2007).

The remainder of the data for waste was based on one waste audit conducted in Rutherford House (URS, 2007). Members of the VUW Environmental Committee ${ }^{9}$ in conjunction with the MfE conducted this waste audit in March 2007. This audit was based on two days of waste collection. The total weight of the waste collected was 235 kilograms. These data were then extrapolated for the whole of the University (URS, 2007).

The Rutherford House waste audit found that $62 \%$ of the waste was recyclable and a further $26 \%$ could be composted (McQuillan, 2007). Assumptions were then made in the URS Environmental Audit for all waste produced by the entire university (URS, 2007). This assumption could bias the final estimate for the GHG emissions for waste, although the direction of any bias is not known. For a more accurate estimate, the university can improve the record keeping of waste recycled and waste sent to landfill. Further waste audits are needed for a more accurate understanding of the composition of waste going to landfill.

\section{Paper}

VUW uses a large quantity of paper for its operations. Data on the amount of paper used at VUW was based on the actual amount of paper purchased in 2006 by the university. While paper usage

\footnotetext{
${ }^{9}$ The author took part in the waste audit at Rutherford House.
} 
is a Scope III emission, the emissions resulting from its use are four times as much as the emissions resulting from fuel used by the university fleet (Scope I).

\section{Emissions not accounted for in the URS GHG audit}

The URS carbon footprint accounts for emissions from energy, transportation, commuter traffic, air travel and solid waste. Emissions produced from agriculture are not accounted for. This is due to limited data in this area (pers. comm. Wilks, 2007). Emissions from agriculture include fertiliser application on fields and grounds. Fertilisers that contain nitrogen inevitably release nitrous oxides into the atmosphere. Further improvements can be made in this area with systems in place that record this information. Emissions from toxic waste were not accounted for. Several student hostels not directly owned by VUW and rooms owned by the VUW, but leased to private businesses, were not included in the URS audit, as these fell out of the range of the university operational boundary.

\subsubsection{Cost to neutralise 2006 GHG emissions}

CarboNZero has estimated that $\$ 330000$ is needed to neutralise VUW's 2006 GHG emissions. This includes measuring, mitigating and certifying VUW's carbon neutral status. Excluding electricity, the GHG footprint for VUW in 2006 was approximately 12,400 tonnes of $\mathrm{CO}_{2} \mathrm{e}$. To get the cost per tonne, fixed costs must be subtracted from the total cost, and then divide by tonnes.

$$
\begin{aligned}
\text { Cost per tonne } & =(\text { total cost }- \text { fixed costs }) / \text { tonnes } \\
& =(329,200-75,000) / 12,400 \\
& =\$ 20.50 \text { per tonne }
\end{aligned}
$$

It will cost around $\$ 20.50$ per tonne of $\mathrm{CO}_{2}$ e to offset 12,400 tonnes through carboNZero. This figure can be compared to a Treasury figure for $\mathrm{CO}_{2} \mathrm{e}$ of US $\$ 11.90$, or approximately $\mathrm{NZ} \$ 15$ per tonne of $\mathrm{CO}_{2} \mathrm{e}$ (Treasury, 2007: 4). Treasury recommends that this figure be used to estimate a carbon price for the purpose of recording the Kyoto-related liability for New Zealand.

Using the figure of \$15 a tonne, the cost to offset VUW's GHG footprint for 2006 will be approximately $\$ 186,000$, compared to $\$ 254,200$ to purchase offsets through carboNZero. 
Although the cost of mitigation through carboNZero is higher than purchasing Kyoto offsets, it has to be noted that offsets purchases on the voluntary market are typically cheaper than offsets purchased on the Kyoto market. If VUW chose to become carbon neutral by purchasing offsets through another means, as opposed to becoming carbon neutral through carboNZero, this could save the university a considerable amount of money. Either way, in order to have environmental integrity, VUW will have to purchase verified offsets that meet voluntary standards and industry guidelines, as discussed in Section 2.1.2.

\subsubsection{Comparison of 2006 and 2007}

The GHG footprint difference between the URS estimate for 2006 and the in-house estimate by VUW for 2007 appears large, 24,700 in 2006 compared to 14,800 tonnes in 2007. However, using an average emission factor for electricity, instead of a marginal emission factor for electricity, now makes the two estimates comparable.

Table 11: Comparison of 2006/2007 VUW GHG Footprint (including electricity) (URS, 2007; pers. comm. Wilks, 2008)

\begin{tabular}{|c|c|}
\hline $2006\left(\mathrm{CO}_{2}\right.$ tonnes $)$ & $2007\left(\mathrm{CO}_{2}\right.$ tonnes $)$ \\
\hline$\approx 16,900$ & $\approx 14,800$ \\
\hline
\end{tabular}

This shows that emissions produced by VUW in 2007 decreased by 2000 tonnes from 2006.

Table 12: Comparison of 2006 / 2006 VUW emissions profile

\begin{tabular}{|c|c|c|}
\cline { 2 - 3 } \multicolumn{1}{c|}{} & $2006\left(\mathrm{CO}_{2}\right.$ tonnes $)$ & $2007\left(\mathrm{CO}_{2}\right.$ tonnes $)$ \\
\hline Business Travel & 3233 & 1906 \\
\hline Commuting Students $^{*}$ & 3830 & 3830 \\
\hline Commuting Staff $^{*}$ & 1291 & 1291 \\
\hline Electricity & 4502 & 4095 \\
\hline Gas & 3328 & 3063 \\
\hline Diesel Generators & 2.27 & 282 \\
\hline Waste & 292 & 280 \\
\hline Paper & 456 & 14748 \\
\hline Total & 16934 & 1.20 \\
\hline
\end{tabular}

* The 2006 commuting figures are based the 2007 travel survey. 
Over the period 2006 to 2007, VUW's GHG footprint decreased in size, the largest decrease coming from business travel. Although this is a positive signal, the 2006 GHG emissions caused by air travel were estimated, whereas in 2007 actual flight distance in kilometres was recorded (pers. comm. Wilks, 2008).

The decrease in energy emissions was partly due to the implementation of an energy programme in 2007, the installation of energy-efficient mechanical plant, the improvement of the control of heating and ventilation systems, the redesign of lighting systems and targeted awareness campaigns, and a printing policy which drove a change to double-sided default printing (pers. comm. Wilks, 2008).

\subsection{Summary}

URS estimated VUW's GHG footprint for 2006 to be approximately 24,000 tonnes of $\mathrm{CO}_{2} \mathrm{e}$. CarboNZero estimated that the cost to offset this and thereby render VUW carbon neutral was approximately $\$ 330,000$ for one year. This cost would when decrease each year, depending on VUW's yearly GHG footprint. Using the NZBCSD's emissions calculating tool, another estimate of 12,500 tonnes of $\mathrm{CO}_{2} \mathrm{e}$ was calculated. The difference between the two estimations is explained by the different sources included in the measurement and the different emission factor used. The GHG footprint for VUW in 2007 was estimated by VUW to be 15,000 tonnes of $\mathrm{CO}_{2} \mathrm{e}$. 


\section{Can VUW become Carbon Neutral?}

This chapter presents the results from the interviewees conducted amongst the VUW stakeholders to assess whether VUW is in a position to adopt the goal of carbon neutrality. The chapter outlines the perceived opportunities, benefits and institutional barriers that exist for VUW to effectively implement this goal. The opportunities and benefits identified from the interviews conducted will draw upon the literature on the concepts of the enlightened self-interest and shortterm rationales for environmental action discussed in Section 3.4.1 and 3.4.2. The barriers identified by interviewees will be analysed in the terms of the literature on organisational and industrial barriers discussed in Section 3.4.3.

\subsection{Results from Interviews}

The results below are collated from the interviews conducted with the various VUW stakeholders:

\subsubsection{Knowledge of climate change}

Interviewees all agreed that anthropogenic climate change was an urgent and important issue. The interviewees claiming a high level of knowledge on climate change had a better understanding of what carbon neutrality meant, while the interviewees claiming a low level of knowledge on climate change had a poorer understanding the meaning of carbon neutrality.

\subsubsection{Awareness of climate change teaching and research at VUW}

Interviewees believed that VUW demonstrated willingness to address climate change and were aware that VUW already incorporates climate change in its teaching and research. Some interviewees mentioned the new Climate Change Research Institute as an example of this willingness. However, many interviewees were unaware of the extent to which VUW lectures incorporated climate change into university courses and research. 


\subsubsection{An achievable goal}

Interviewees agreed that carbon neutrality was an achievable goal for VUW; yet this was considered to be dependent on the benefits, opportunities and barriers. In the following subsections, the strategic benefits, opportunities and barriers of a carbon neutral initiative identified by the interviewees will be reviewed.

\subsubsection{Benefits and Opportunities identified by the interviewees}

The following results were identified as the benefits and opportunities of a carbon neutral initiative for VUW. Coding and categorising the responses gathered from the interviews into main conceptual areas gave rise to the following categories. These have been further categorised into either examples of short-term interest or enlightened self-interest, as explained in Chapter 3.

The following have be categorised as examples of short-term interest benefits:

- Marketing and branding opportunities

- Cost-savings opportunities

- Opportunities through voluntary activities

The following have been categorised as examples of enlightened self-interest benefits:

- Leadership benefits

- Environmental benefits

- Benefits from working with local / central government

- Moral / ethical benefits

\section{$\underline{\text { Short-term interest benefits }}$}

\section{Marketing and branding opportunities}

Most interviewees identified the marketing and branding opportunities of a carbon neutral initiative as a benefit. Carbon neutrality was seen as a selling point for VUW amongst both prospective domestic and international students. At present, no university in Australasia has implemented a carbon neutrality programme. By becoming the first carbon neutral university in 
Australasia, VUW could experience 'marketing spin offs' (pers. comm. Wilks, 2007), and be seen as a pioneer and market leader. Becoming the first carbon neutral university can be advantageous in a similar way to the benefits for Meridian Energy, who found a competitive advantage by becoming New Zealand's first carbon neutral certified energy provider.

"With the attention that environmental awareness has worldwide, international students may prefer to go to an institution that has taken a positive step to become environmentally sound" (pers. comm. Fontanier, 2007). In the climate of growing competition amongst universities worldwide, carbon neutrality may become an essential selling point to both students and other stakeholders. It is possible that students from North America for example may expect universities to account for their emissions, due to the number of universities signing up to the ACUPCC.

A ShapeNZ survey occurring from August 2006 to February 2007 surveyed 3088 New Zealand consumers (ShapeNZ, 2007). Those surveyed overwhelmingly agreed or strongly agreed that a company's environmental practices have an influence on whether they buy their products or not. It indicates that New Zealanders base their choices on issues including, but not limited to, "green issues". VUW can seize the opportunity this presents by becoming carbon neutral and use the branding that accompanies it. By becoming carbon neutral "there will definitely be benefits around" VUW's "brand and therefore consequential benefits in the attraction of high quality students" (pers. comm. Bentley, 2007).

\section{Cost saving opportunities}

It was recognised that implementing a carbon neutral initiative will lead to financial opportunities for the university, by improving energy efficiency and waste reduction. This opportunity is evident at universities that are implementing emission reduction programmes, as discussed in Chapter 4.

\section{Opportunities through voluntary actions}

It was recognised by most interviewees that there would be opportunities through voluntary actions. It was noted that voluntary actions could be more cost effective than actions that occur because of legislation. Interviewees felt that it was inevitable that the university would be carbon neutral in the future, as the government has announced its intentions for the country to become carbon neutral. 


\section{Enlightened self-interest benefits}

\section{Leadership}

All interviewees identified leadership opportunities for VUW if a carbon neutral initiative was implemented. It is seen that VUW can serve as a model for other universities and organisations. This view of leadership is in line with Agenda21, Chapter 36. Universities are in the privileged position, where they can take a leadership role, through teaching, research, policy development, and demonstrating the principles of awareness and stewardship of sustainability (Dahle and Neumayer, 2001).

\section{Environmental benefits}

All interviewees recognised the environmental benefits for the climatic system as a whole, if the university implements a carbon neutral initiative. However, it was pointed out that this benefit should not be 'exaggerated', as the university's GHG footprint is minuscule in comparison to total anthropogenic GHG emissions (pers. comm. Boston, 2007).

\section{Working with local / central government}

Many interviewees saw that VUW could develop partnerships with both local and central government, and this could create opportunities for both the university community and the wider public. A key objective in the university's strategic plan is that of being linked to the city (VUW, 2006b). The VUW strategic plan gives consideration to programmes that integrate the university with the Capital City and also to the public sector. A carbon neutrality policy will be a good fit for VUW within Wellington, particularly since Wellington City Council announced in December 2007 that it has set a carbon neutral target for its corporate operations by 2012 .

Accounting, reducing and offsetting the university's GHG emissions will also enable it to engage with the New Zealand Government's Emissions Trading Scheme. 'The Emissions Trading Scheme will have financial ramifications for the university, through its energy costs. A carbon neutral initiative will help reduce these costs' (pers. comm. Boston, 2007).

The New Zealand government is already implementing a programme of carbon neutrality for the state sector by 2012, and for the whole of the country to become carbon neutral in the future. 
Adopting a carbon neutral target would not only fit the needs of the key stakeholders represented within the Environmental Committee, but also anticipate future changes taking place in the country more widely.

\section{Moral and ethical benefits}

Several interviewees identified the moral and ethical benefits from becoming carbon neutral. This can be classed into the enlighten self-interest rationale. By 'doing the right thing', there would be several external and internal benefits for the university. The wider community would view the university in a positive light and the university community would feel better about being linked to an ethical university.

\subsubsection{Barriers identified by the interviewees}

Several barriers exist, which hinder the university adopting a carbon neutral objective. However, with each barrier, an opportunity arises to develop new values, create and identify new methods of implementation and create procedures to accommodate a new policy direction. The following results refer to which factors the interviewees considered the barriers hindering VUW becoming carbon neutral. Coding and categorising the responses gathered from the interviews into main conceptual areas gave rise to the following categories. These barriers have been further categorised into either industrial or operational barrier, as explained in Chapter 3.

The following barriers can be categorised as industrial barriers:

- Financial

- Higher priority of other initiatives

- Information system barriers

- Academic priorities

- Education and awareness

The following barriers can be categorised as organisation barriers:

- Lack of support or commitment

- Cultural and behavioural barriers

- Lack of space 


\section{$\underline{\text { Industrial barriers }}$}

\section{The lack of financial resources}

The results indicate that the main barrier to implementing a carbon neutral programme at VUW is the cost of funding it. It was generally asserted that carbon neutrality was a preferred goal; however VUW will need to conduct careful budgetary planning to achieve it, and if the costs were too great, then carbon neutrality would remain a future goal for VUW, until it became affordable.

On the 14 December 2006, the Minister for Tertiary Education, the Hon. Dr Michael Cullen, released the second Tertiary Education Strategy. The strategy incorporates Statements of Tertiary Education Priorities 2008 - 2010. “The government announced a new approach to tertiary funding. Government expenditure on tertiary education will no longer be 'demand driven', but instead will be set as a three-year funding path" (TEC, 2006). This will see a change from funding per student to bulk funding. The focus is now placed research and teaching outcomes.

This funding change will effectively place a barrier in the way of VUW becoming carbon neutral. Adequate funding is a restriction on all university priorities. It has been projected that VUW may now have an excess of around 1000 students in 2008, in terms of funding allocation - relative to its funding cap (pers. comm. Wilks, 2007). This alone will place limits on finances available to support learning and teaching, let alone other objectives.

\section{Higher priority of other initiatives}

There was agreement amongst the interviewees that the number one priority for the university is to provide a climate where world-class research and teaching can occur. This is in line with the university's strategic plan, which sets out an objective that the University is one of the top two research-led universities in the 2012 PBRF evaluation (VUW, 2004).

Another priority identified by some interviewees was the Campus Development Framework (VUW, 2006c). The period 2006 to 2016 will see the university redevelop several parts of the Kelburn campus, including:

- The Fairlie Terrace student accommodation complex and Laby Building Extension. The funding for these projects has already been approved and construction is under way. 
- The Campus Hub centred on the Rankine Brown Building and the Quad. This will be a major reconstruction project. Funding for this is yet to be approved.

The limited funding from TEC and the Ministry of Tertiary Education will see the university having to make stricter choices in terms of investment. As the manager of Facilities Management put it "the campus hub is an investment that will almost have to be done, it will result in the university placing less emphasis on carbon neutrality as a major objective" (pers. comm. Bentley, 2007).

\section{Information system barriers}

The lack of a comprehensive and integrated information system is considered a significant barrier, as the record-keeping systems have not been in place in previous years. Therefore, it is difficult to understand the university's GHG footprint prior to 2006. Therefore, it is difficult to predict any emission reductions scenarios for future years based on previous years' emissions prior to 2006

\section{Academic priorities}

It was recognised that academic priorities pose a barrier. For instance, the desire to reduce student-to-staff ratios and make headway in addressing the gap between domestic and international salaries for academic staff was noted as important academic priorities, which were placed before funding a carbon neutral initiative.

Another component of academia is travelling to conferences and other universities to conduct research. Business travel represents a large component of the VUW GHG footprint, and this may pose a problem, as academics may not be willing to stop or minimise travelling overseas.

\section{Education and awareness}

Education and awareness were identified as a barrier and the consensus was that there needs to be a university community-wide education and awareness campaign before successfully implementing a carbon neutral initiative at VUW. This campaign can help generate more of a buy-in into the initiative. 


\section{Organisational barriers}

\section{Lack of support or commitment}

All interviewees noted that there needed to be a commitment by the University Council and, in particular, the Vice-Chancellor, for a carbon neutral initiative. Without this commitment, carbon neutrality would not happen at VUW. It was also noted that there had to be general support from the entire university community if a carbon neutral initiative was to be successful.

\section{Cultural and Behavioural barriers}

The majority of interviewees considered cultural and behavioural change as a major barrier to overcome. It is possible to become carbon neutral by simply purchasing offsets every year to balance the university's carbon footprint; thus a cultural and behavioural change would not need to occur. However it was acknowledged that attitude and behavioural change would aid oncampus emission reductions, therefore reducing the cost of purchasing offsets. Ultimately, lasting sustainable behavioral change is the real goal to meeting the challenge that climate change presents (pers. comm. Barker, 2007).

\section{Lack of space}

Another barrier identified was the lack of space at VUW. This was seen as a barrier to becoming carbon neutral. For example, if the university intended to increase its carbon sinks, it would not be possible to do so through planting trees, unless the university purchased more land, with the intent to plant a large-scale forest. The lack of space is such a problem that there is no room to for an on-campus compost facility, which would be ideal to help reduce the GHG footprint from waste (pers. comm. Bentley, 2007).

\subsection{Summary}

Carbon neutrality is viewed by interviewees as a feasible initiative for VUW. The interviewees identified many enlightened self-interest and short-term opportunities and benefits for VUW that would be gained by implementing a carbon neutral initiative. However, the success of this initiative is dependent on overcoming several existing barriers, including financial constraints and lack of commitment from the University Council. 


\section{A Framework for Implementing a Carbon Neutral Initiative at VUW}

The theory behind the implementation of carbon neutral initiatives at universities is relatively new and still developing. This chapter therefore analyses why, both in theory and practice, a particular method might be chosen when designing and implementing a carbon neutral initiative. By understanding the economic, environmental and social reasoning behind this method, the future designers of a carbon neutral initiative will be better equipped to make informed decisions. The carbon neutral initiative framework in Figure 17 will be used. The framework has been developed from both the theory and information gathered from the interviews:

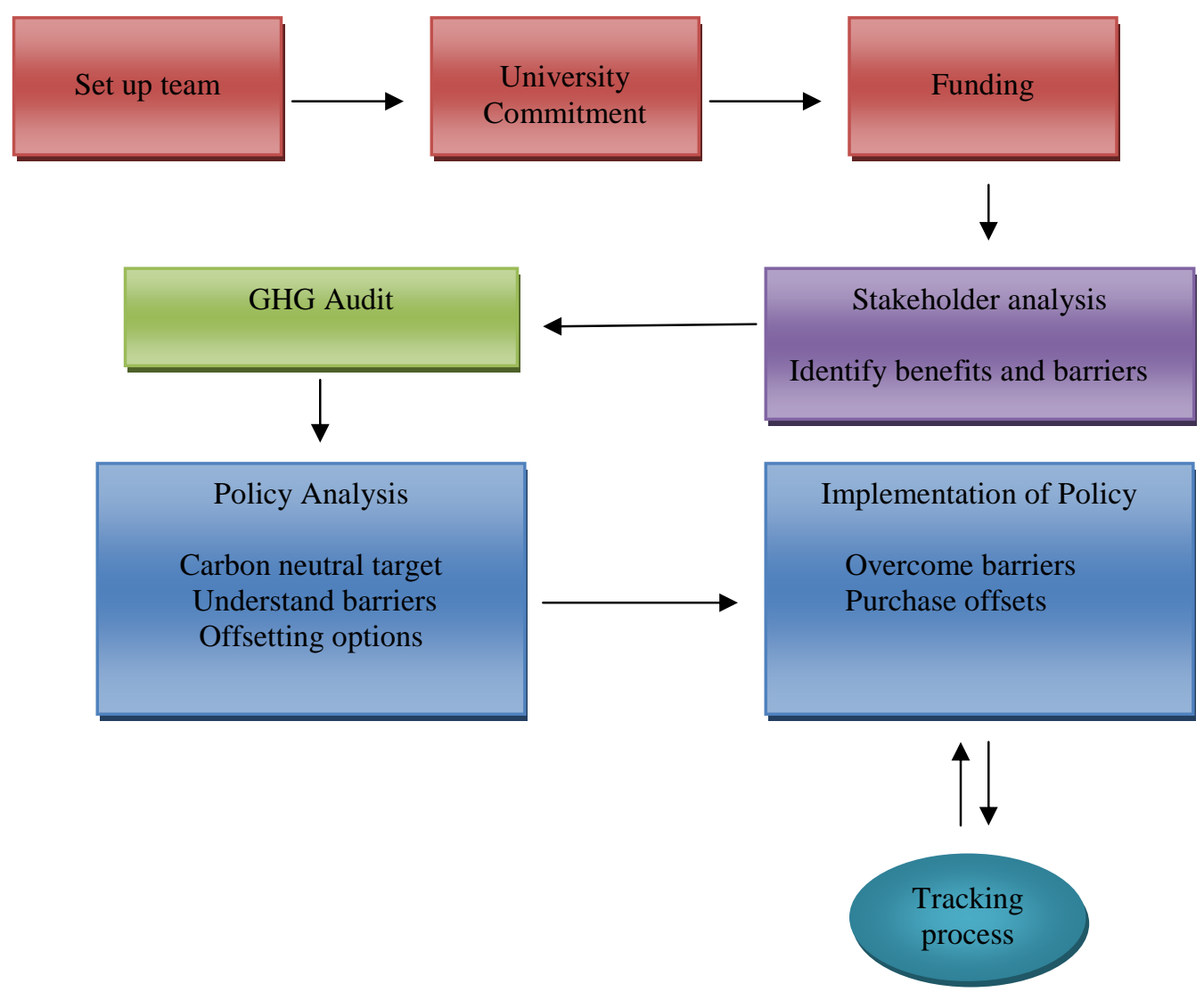

Figure 17: Carbon neutral initiative framework

From the literature, an environmental initiative involves a series of consecutive steps (Creighton, 1998; Sharp, 2002; Clarke, 2006). The process begins with forming a team that steers the project. The next step involves obtaining support and a commitment from senior management, or in the case of a university, the university council. The team then has to arrange adequate funding 
for the project. The next stage involves policy development. A stakeholder analysis should be conducted and the policy be designed to meet the needs of the stakeholders. Following this, the organisation plans for the policy's implementation, implements the policy, monitors outcomes and then reviews the policy accordingly. The section below will present both a theoretical and practical plan for a carbon neutral initiative at VUW.

\subsection{Results from interviewees}

The following results refer to which factors the interviewees believe are needed for the process of implementing a carbon neutral initiative. Coding and categorising the responses gathered from the interviews into main conceptual areas gave rise to the following categories:

- Dedicated team

- University commitment

- Funding

- Stakeholder analysis

- Inventory

- Policy analysis and design

- Implementation of policy

- Tracking progress

\section{Setting up a carbon neutral team}

An initial step by organisations that implement successful environmental initiatives, including a carbon neutral initiative, is the formation of a dedicated environmental steering committee (Strachan, 1996; Creighton, 1998; Clarke, 2006). To be successful, the committee must include a person from senior management, who can provide direction and answer questions about the organisation that others on the committee may ask (pers. comm. Hutton, 2007). This is important for two reasons. First, it allows for environmental initiatives to be factored into wider management issues, e.g. marketing and the social branding aspirations of universities. Second, through involvement, the financial, social and environmental benefits will become more visible at senior levels of the university. This is likely to underline the importance of such initiatives and lead to continued support (Carpenter and Meehan, 2002; Sharp, 2002; Bekessy, et al, 2007). 
The committee should also be made up of other stakeholders within the organisation who are prepared to be committed to the project and determined to carry it forward (Hutton, 2007). However, the committee must also have flexibility, as the process can take time, while committee members such as representatives from the student body might only be able to commit for a short period.

Ideally, one or more committee members should be environmental leaders or champions. The campus sustainability literature underlines the need for environmental leaders on environmental committees (Creighton, 1998; Clugston and Calder, 1999; Walton et al, 2000; Shriberg, 2002). For example, Walton et al. (2000: 524) found that there is normally an active environmental champion involved in the formation of the policy and its implementation in institutions that have implemented successful environmental initiatives.

\section{University commitment}

To successfully achieve environmental initiatives, and in particular carbon neutrality, it is widely acknowledged that there needs to be visible and meaningful commitment from high level university executives (Creighton, 1998; Clugston and Calder, 1999; Bekessy, 2007; Hutton, 2007; Yarr, 2007; Shand, 2007). Chernushenko (1996: 4) summarises the driving forces from senior management as follows:

\footnotetext{
"A good structure for campus environmental management takes a simultaneous top-down and bottom-up approach. First, support for this significant shift to sustainable practices must come from the top. Not only must the most senior people be interested in the cause, they must be seen to be so. They must be 'champions' of the cause, showing vision and leadership. Second, people throughout the organisation must be a part of such an initiative. They need to believe that they have an equal stake in achieving better environmental management and that they will share in the benefits. This requires that all members of the organisation be involved in the development, implementation, monitoring and enforcement of the initiative".
}

However, it is also acknowledged that this commitment is absent at most universities for many environmental initiatives (Thompson and Green, 2005; Bekessy, et al, 2007). In this case, some researchers suggest that environmental initiatives have to be developed 'that do not assume a topdown approach' (Thompson and Green, 2005: 8). However, this is clearly not the case when it comes to a successful carbon neutral initiative, as this needs both high-level and other support and requires university-wide change. 
The requirement of high level commitment for a successful carbon neutral programme is recognised by VUW Environmental Manager, Andrew Wilks: "There will be different levels of responsibility involved. Although the lead will come from the official working team, which includes a high level manager, in pushing the initiative forward, a commitment will have to come from the senior management team, who are the next level down in the process" (pers. comm. Wilks, 2007). It is also clear that at a very high level, in the University Council, there is considerable support for carbon neutrality (Beaglehole, Speech, 2006; Daugherty, 2007; Duggan, 2007).

\section{Funding}

Interviewees indicated that funding was vital for the success of a carbon neutral initiative. This is in line with the literature. Beekeesy, Samson and Clarkson (2007) found that a long-term budget is needed for the success of campus environmental programmes. This is to ensure that resources are available to enable universities to meet commitments. Without adequate funding, any response will most likely fail.

The development, implementation, monitoring and enforcement of a carbon neutral initiative at VUW will require adequate funding. For example, as discussed in Section 5.2, it was estimated that VUW can go through the carboNZero certification programme and can become carbon neutral for approximately $\$ 330000$ for the first year. Other options such as on-campus emission reduction projects will also require up-front funding. As discussed in Section 6.1.5, a major barrier to VUW becoming carbon neutral is the financial costs. Funding will have to be sourced from somewhere; some options (as suggested by the interviewees) for funding may include the following:

\section{The Vice-Chancellor}

The Vice-Chancellor may be able to fund the entire initiative through the Vice-Chancellor's discretionary budget. This would require full support and commitment from the Vice-Chancellor and the University Council (pers. comms. Wilks, 2007; Bentley, 2007; Barker, 2007; Boston, 2007; Duggan, 2007). There would, of course, be an opportunity cost, against which benefits of carbon neutrality would have to be weighed. 


\section{Student Fees}

It was suggested that if there is enough support from students, funds can be raised through student fees (pers. comm. Barker, 2007). For example, in 2006 there were 21,076 students enrolled at VUW (VUW, 2006). If there was full financial support from students, VUW could become carbon neutral through the carboNZero programme, if each student was willing to contribute at the rate of one dollar each week during trimester one and two, or a total of around $\$ 24$ per calendar for the entire year. Costs are likely to fall in subsequent years.

\section{Alumni}

Approaching alumni may provide a useful resource for both funding opportunities and expertise in emission reductions. Carefully designed projects may appeal to alumni's sense of loyalty towards the university (pers. comm. Barker, 2007).

\section{Central / Local government}

With the government's target of the country one day becoming carbon neutral, lobbying the government for a contribution, such as a seeding grant, may be an effective means to provide the funding needed for the carbon neutral transition (pers. comm. Shand, 2007).

The Wellington City Council's goal of carbon neutrality also provides an opportunity for funding. By working with the council, the university can provide expertise in areas of renewable energy technology, energy efficiency and green building design, while the council may be able to provide the financial backing needed (pers. comm. Shearer, 2007).

\section{External Funding Organisation}

Sourcing funding from external funding organisations may be successful, as this can benefit both the university and the external funder. Partnering with business and industry leaders to deliver joint projects can benefit local industry by promoting Wellington as a sustainable business centre. Businesses willing to provide funding may be able to claim some 'enlightened self-interest' benefits (pers. comms. Wilks, 2007; Barker, 2007). 


\section{Stakeholder analysis}

Before environmental initiatives can be successfully implemented, the complex nature of a university has to be understood and addressed. The multi-layered, complex nature of a university presents no single pre-eminent observation point or any single control-centre from which university-wide changes can be made (Sharp, 2002). A carbon neutral objective for VUW will involve a mixture of circumstance, interests, willingness and opportunities. However, the empirical case study evidence on sustainability in higher education signifies the importance of involving stakeholder's as active and effective agents for change (Shriberg, 2002).

A stakeholder analysis is a useful method to understand how people in the university community are affected by the implementation of a carbon neutral initiative (Grimble and Wellard, 1997). They may be directly or indirectly affected and the initiative may be beneficial or costly for them. An analysis allows for the identification of the co-benefits and co-costs involved, and provide an improved assessment of the efficiency of the initiative (Grimble and Wellard, 1997).

\section{Auditing / Inventory}

For any organisation such as VUW to achieve the status of carbon neutrality it has to first understand its carbon footprint on a yearly basis - both in size and what influences the size. As discussed in Section 3.5, a GHG emissions audit gives this understanding. Additionally, conducting an audit is fundamental for a valid GHG reduction strategy, as it is a signal of organisational commitment to confronting the climate change challenge.

Accounting for GHG emissions has allowed VUW to identify the biggest sources of emissions and the most effective reduction opportunities. Ideally, the top priorities are areas where the net costs per tonne of $\mathrm{CO}_{2} \mathrm{e}$ abated are lowest. This will depend on both the direct cost of reductions (e.g. implementation costs) and co-benefits (e.g. improved student or staff comfort). These areas can then be targeted (The GHG Protocol, 2004). After conducting a stakeholder analysis and GHG audit, a carbon neutral policy can be designed from the resulting information. 


\section{Policy analysis and design}

A GHG audit on VUW has already been conducted for 2006 and 2007. Combining this with a stakeholder analysis will allow for VUW to begin analysing and designing an appropriate carbon neutral policy, one that fits the needs of VUW's community.

\section{$\underline{\text { Introduce and analyse a carbon neutral target }}$}

Interviewees were in agreement that VUW should commit to a timeframe of carbon neutrality, as opposed to simply purchasing offsets every year to match the university's carbon footprint. This means that the focus of the carbon neutral initiative will be on emission reductions. This will involve developing mitigation strategies and conducting a feasibility study. VUW can follow four scenarios, similar to those outlined in the report commissioned by The Rocky Mountain Institute for Oberlin College (Heede and Swisher, 2002: 74). A cost benefit analysis will have to be conducted to see which scenario will achieve a carbon reduction objective at the lowest possible cost. The principles are:

- The offset purchase scenario

VUW can continue emitting without investing in any on-campus emission reductions programmes or behavioural change programmes. Carbon neutrality can be achieved simply by purchasing offsets every year to balance out its carbon footprint. This option may however become costly over a few years with no emission reductions programme, especially if emissions or carbon credits increase in price over that time. Interviewees were not in favour of VUW simply purchasing offsets to balance its GHG footprint.

- The "no-brainer" (low-hanging fruit) scenario

This scenario involves simple, low-cost emission reduction programmes and behavioural change programmes, with any emissions that cannot be reduced simply being offset. This option places a greater emphasis on buying offsets, as opposed to emission reductions. It would require little effort from the university community and emissions may still rise over the years. However, the only issue with choosing this option is that 'VUW has already tackled all low-cost emission reductions, and it appears that any more emission reductions may need a large amount of financial capital invested into it' (pers. comm. Wilks, 2007). 
- The "no-regrets" scenario

In terms of emission reduction benefits, much research has been devoted to analysing what has been termed 'no-regrets' emissions reduction (Marechal, 2007). Emissions reduction is considered no-regret when the cost of implementing measures is outweighed by the financial benefits gained. This scenario would require a large investment into emission reduction initiatives and behavioural change programmes, as opposed to buying offsets.

A component of the university's GHG footprint is caused by travel, both business and commuter, and reducing this may pose a problem. One of the barriers identified in the last chapter was the difficulty of reducing GHG emissions associated with academic travel overseas. While some travel can be avoided, offsets may have to be purchased to offset most business travel to achieve carbon neutrality.

- The "no-prisoners" (carbon neutral) scenario

This scenario would require that the university aim to reach a carbon neutral target with emission reductions and without the purchase of any third-party offsets. This would require on-campus sources of emissions and on-campus sinks or sinks owned by VUW to be equal. Therefore, a significant investment would have to be made. Options include investment in renewable energy projects or purchase of land to plant forestry. If the university invested enough capital into this, it may be able to sell any excess credits.

As mentioned in the last chapter, a major barrier to achieving carbon neutrality is the financial constraints, therefore this scenario is unlikely to be implemented in the short-term, as it will require a large amount of up-front capital, and returns may take years. However, with the longer-term prospect of ever-more stringent emission reduction goals and measures being needed as climate change worsens.

\section{Implementing the policy}

Once a policy has been agreed on, it will then have to be implemented. Depending on which scenario is chosen, it will most likely have to include behavioural change programmes, such as education programmes for staff ands students. While educating the university community may lead to changes in values and ethics, this does not necessarily mean changes in behaviour 
(McKenzie-Mohr and Smith, 1999). Unfortunately, a variety of studies have established that enhancing knowledge and creating supportive attitudes has little or no impact upon behaviour change (McKenzie-Mohr, 2000).

For changes in behaviour to occur, community-based social marketing should be used (pers. comm. Boston, 2007). Community-based social marketing is comprised of four steps: uncovering barriers to behaviours and then, based upon this information, selecting which behaviour to promote; designing a programme to overcome the barriers to the desired behaviour; piloting the programme; and then evaluating it once it is broadly implemented (McKenzie-Mohr, 2000: 546). Successful implementation of GHG mitigation polices depends on smooth integration into existing efforts towards campus sustainability and this may take a considerable period of time (pers. comm. Wilks, 2007).

\section{Track and review progress}

Once a target and mitigation strategy is chosen, it will be necessary to track performance in order to assess whether the target is correct and if the mitigation strategy is on the right track. If there are any problems with either, then they will have to be adjusted to suit (The GHG Protocol, 2004).

Despite the expense of delivering many environmental programmes, evaluation of their effectiveness are infrequent. Community-based social marketing stresses the evaluation of implemented programmes. Additionally, it emphasises the direct measurement of behaviour or its consequences (e.g. tracking emissions from energy usage or commuting) as opposed to relying on self-reporting (McKenzie-Mohr, 2000: 549).

Tracking emissions over time will allow the university to meet several goals, including public reporting, establishing targets, managing risks and opportunities, and being able to meet the needs of various stakeholders. Any new buildings, changes in fuel or energy sources, or other factors can also be accounted for (The GHG Protocol, 2004). One issue with VUW tracking emissions over time is that complete record keeping has not occurred in the past and 2006 is the first year in which VUW could collate enough complete data to estimate a GHG footprint, and even this is limited. This GHG footprint will become more accurate as the record-keeping process improves (pers. comm. Wilks, 2007). 


\subsection{Summary}

The interviewees from both VUW and organisations that have already committed to a carbon neutral initiative identified a series of consecutive steps, in regards to the implementation of a carbon neutral initiative at VUW. These steps are consistent with the literature, and include establishing a dedicated team to designing the initiative, getting a commitment from the University Council, arranging adequate funding for the project, conducting a stakeholder analysis to identify barriers, conducting a GHG audit, designing an appropriate mitigation strategy, implement this strategy, and then tracking and reviewing progress. 


\section{Discussion}

The IPCC states that industrialised countries GHG emissions need to be reduced by 60 to 80 percent by the year 2050 in order to avoid catastrophic climate change (IPCC, 2007d). Climate scientist Jim Hansen (2008) argues that we need to go further, reducing current $\mathrm{CO}_{2}$ concentrations to $350 \mathrm{ppm}$. This will require a global effort to meet stringent targets and advance new ways of thinking. It will take comprehensive research, whole systems thinking, technological innovation and a higher degree of environmental literacy amongst academics, political leaders and the general population. To achieve this, there has to be co-operation from the higher learning sector and it will be necessary for universities to take a lead role in guiding humanity through the impending climatic crisis. Mitigation of anthropogenic climate change requires rapid and widereaching action from the higher education sector far beyond current levels.

Despite incorporating climate change into much of its teachings and research, VUW still lags behind many international universities in the way it deals with its own GHG emissions; this is evidenced in Chapter 4. Although VUW began implementing an environmental policy, it is yet to implement a policy designed specifically to manage and reduce GHG emissions produced by its operations. However, carbon neutrality is a viable goal within the scope of the VUW Environmental Policy - in particular, the commitment to sound business practices, community leadership and management of the university's environmental footprint.

There are a number of reasons to adopt carbon neutrality as a target. Firstly, the evidence presented in Chapter 2 concludes that worldwide emission reductions are necessary if the serious consequences of anthropogenic climate change are to be avoided. Only achieving carbon neutrality at a global scale will see the concentration of GHGs in the atmosphere stabilise and reduce to safe levels. New Zealand will need to be part of such a global transformation. By adopting a carbon neutral target, VUW can play its part in mitigating climate change, demonstrating leadership and integrity and preparing itself for the risk imposed by future government regulations.

Secondly, by accounting for both sources and sinks rather than focusing merely on a percentage reduction in emissions, carbon neutrality takes a broader perspective on the basis for the development of ongoing policy. By reducing GHG emissions as much as possible, and then offsetting emissions that cannot be reduced, the university can avoid having a net impact on the 
concentrations of GHGs into the atmosphere. With regard to percentage reductions in emissions, it has to be decided when and where a baseline is to be established. All emission reductions have then to be set in accordance with this baseline. As indicated by the interviewees, VUW plans to grow in size and build new buildings. Carbon emissions management initiatives involving percentage emission reductions complicate such situations, whereas, carbon neutrality requires only that sources and sinks are in balance.

Committing to carbon neutrality would not only be an ideal way to manage GHG emissions but also create many benefits and opportunities for the university, as identified in Chapter 6. However, several barriers will need to be overcome before this commitment can be implemented.

\subsection{The International Context}

As part of the investigation, the actions of other universities in response to climate change were researched. It is clear that numerous universities are responding to the societal demand for action on climate change by making attempts to address carbon neutrality in their campus operations. Universities in the USA and the UK have already made advances toward reducing their GHG emissions. Although this has only been a recent development, several universities in the USA have committed to carbon neutrality through the ACUPCC, and St. Andrews University in Scotland is currently investigating its options before making this commitment.

By taking the initial steps towards managing GHG emissions, these universities are exhibiting the leadership needed to guide society. As previously mentioned, the ACUPCC pledge's aim is for universities and colleges to exercise leadership in their communities and throughout society by modelling ways to eliminate GHG emissions.

This is a critical step in being able to mitigate global GHG emissions because it demonstrates that such action is possible and, indeed, potentially beneficial for higher learning institutions. To its credit, the ACUPCC commitment goes beyond simply offsetting emissions, involving a variety of options to reduce and manage emissions. By committing to such a scheme, it is apparent that these universities are acknowledging their role as agents for change. Universities that are managing their emissions are also building and engendering expertise that will assist in directing society towards a low-carbon economy. 
Evidently reducing GHG emissions and committing to the aim of carbon neutrality is gradually becoming standard practice at many universities internationally. This has been demonstrated by the rapid response of American Colleges and Universities signing the ACUPCC pledge over October 2006 to September 2007. Making such a commitment is feasible for VUW, if other universities can adopt a carbon neutral initiative; there is little reason why VUW cannot.

However, there is an emerging understanding, in both the USA and the UK of the importance of collective support. In the USA, universities are working together and sharing ideas and methods to reduce emissions, while in the UK the Carbon Trust is providing funding and expert advice for universities in the UK to manage emissions.

An obstacle to VUW achieving the success of universities overseas is that this direct support is lacking in this country. While it may be argued that carboNZero can partially fulfil this support role, this possibility has not yet been realised and, if it were to be, may lack effectiveness if not tailored to the complex contingencies specific to New Zealand universities. If VUW chooses to become carbon neutral, it must decide whether to do so independently and thus gain the advantages of becoming the first New Zealand University to do so, or else work closely with other universities in order to source collective support.

\subsection{VUW's GHG audit}

A core component of CSR initiatives is that institutions should play a greater role in society by extending their area of concern beyond their bottom line. This goes hand in hand with the idea that universities can play their part as agents of societal change. The current bottom line for universities is research and teaching output. VUW has, however accepted responsibility for its environmental impact, releasing an environmental policy, and is now working to address this impact. The first step made was to complete an environmental audit for its 2006 operations. Since then an audit for its 2007 GHG impact has been completed.

By auditing the university's environmental impact, areas that have a large or easily reduced environmental impact are clearly indicated. This will allow the university to develop the most cost-effective strategies to manage the university's GHG footprint. Depending on lowest cost, emission reduction strategies can be implemented where possible, or offsets can be purchased. 
However, problems arise when studying the audit data. The raw data collected for both the 2006 and 2007 audits had several emission sources missing; the audits were limited in size, scope, or made assumptions regarding particular sources. For example, the waste audit used a small sample, taken over a two-day period, from only one campus.

As shown in the analysis, this was extrapolated to the entire university for the 2006 period, even though the waste audit was performed in early 2007. As the university improves its environmental record keeping, these problems will be overcome and the environmental impact assessment will become more accurate. A flow-on effect will be that certain areas can be targeted and the environmental impact of the university will inevitably be improved. Until better data collection is achieved, a barrier to carbon neutrality exists in the form of incomplete data, implying that carbon management decisions would not be based on full information.

With regard to communicating and understanding VUW's emission impact, the GHG footprint tool can be effective. The improvements made over the 2006 to 2007 period, in terms of the university's GHG impact, will be included in the VUW 2007 Annual Report. This communicates that small improvements can have a significant positive impact upon university operations. This will demonstrate to the University Council and senior management that environmental initiatives can be effective. It may in turn encourage buy-in for further environmental initiatives, such as a commitment to carbon neutrality.

This stated, it should be noted that 'recorded' improvements so far are largely due to better record keeping as opposed to major changes in behaviour, as only limited energy management actions have been taken and VUW is yet to initiate a travel plan (see previous chapter). This is an example of where it is impossible to provide completely accurate emission data, due to uncertainties over the emissions inventory, as discussed in Chapter 3. The VUW audit may correctly record data, but the extent of data needed to complete an accurate model is lacking - and estimation or even guesswork may be needed (Winiwarter and Rypdal, 2001).

The disparity between the official GHG footprint for VUW, conducted by URS, and the GHG footprint obtained by using the NZBCSD assessment tool demonstrates that organisations measuring their GHG footprint need to be aware of the limits of carbon auditing. URS is more comprehensive in its approach, measuring emissions from Scope I, II, and III, and using a marginal emission factor for electricity. The NZBCSD tool measures emissions from Scope I and 
II, and uses an average emission factor for electricity. There is a 100 percent difference between the two GHG footprints calculated.

If an organisation aspires to possess environmental integrity, and avoid being accused of green wash, the GHG Protocol Initiative advises universities to measure the widest range of emissions produced by an organisation's operations as is possible. VUW is beginning to improve its record-keeping systems and so the GHG footprint will, over the next few years, become more accurate. In this case, if VUW does decide to become carbon neutral, it may save money due to not having to purchase carbon offsets to cover inaccurate emissions data.

Emissions produced from transportation form a large component of the university's GHG footprint. Though it is the component that staff and students have the most control over through their individual decisions, this control is limited by a number of factors. These limits include costs, mode, availability, and location of accommodation. Clearly, the university is unable to remove these barriers to more sustainable modes of transport without working closely with local government and the public transport companies. This is a formidable - though not impossible task that in itself constitutes a barrier to carbon neutrality at VUW.

Another issue arising from the results of the VUW GHG audit is that of emissions associated with business travel. A key part of an academic's professional development is furthering knowledge and experience through sabbaticals or conferences. Consequently, a large proportion of VUW's GHG footprint is caused by air travel. To properly manage air travel emissions, several options are available.

Firstly, air travel can be restricted. This option will, most likely, be unpopular, and will have follow-on effects. Academics may refuse to restrict the amount of travel that results from the furtherance of their own education and learning. Other options include investing in and promoting the use of, video conferencing, although this is not always a good substitute for travel. Ultimately, the university has to assist academic staff to develop professionally in less carbonintensive ways. Further studies are desirable, such as surveying academic staff on what options they prefer.

When staff have to travel by air, offsets can be bought to neutralise the resulting emissions produced. However, offsets will increase the cost of travel and these costs will have to be passed 
on to VUW. However, it has to be recognised that, due to international concerns about climate change and the likely rise in the cost of fuel, the costs of international air-travel will continue to steadily increase in future, regardless (Pearce, 2007). Society in general and universities in particular are likely to have to adjust to air travel becoming increasingly expensive. In contrast, video conferencing will reduce costs associated with air travel and reduce GHG emissions otherwise produced from air travel. Financial savings from video conferencing can in principle be used to purchase the carbon offsets needed to neutralise emissions from the air travel that is deemed necessary.

Options to reduce emissions produced by student commuting include more long distance teaching via the internet. This option is limited at the moment, due to the low quality broadband services available in the country. Until broadband service providers improve the speed of broadband in this country, the potential of long distance teaching will continue to be limited.

\subsection{Likelihood of a carbon neutral initiative at VUW}

The conclusion of this thesis is that VUW can become carbon neutral. This decision, however, remains with the Vice Chancellor and / or the University Council, although it is preferably the sort of strategic decision that the Council should make. Whether the University Council believes that the benefits and opportunities of becoming carbon neutral outweigh the costs associated with surmounting the barriers currently in place is the central question. With a CSR model, the perceived benefits, opportunities, and barriers of environmental initiatives can be identified by interviewing various stakeholders at VUW. This provides a wider scope for decision-making, as opposed to basing the decision solely on a financial rationale.

All interviewees were in agreement that VUW can become carbon neutral if certain criteria are met. While those claiming a high level of knowledge on climate change also believed that a carbon neutral goal was an important objective for the university, interviewees claiming a lower level of knowledge on climate change did not believe that it was as high priority for the university as other objectives.

A key step towards the goal of carbon neutrality being achieved is a firm commitment from the University Council or from top level management and then someone from this top level helping 
drive this initiative. This was a repeated theme recognised by the interviewees and from the literature studied in this thesis.

While many people have seen Al Gore's movie, The Inconvenient Truth, and have read media reports on climate change, a surprising number still appear to dispute the reality of climate change. Humanity is yet to adopt patterns of serious thought and action needed to combat anthropogenic climate change. A widespread information campaign detailing reasons why emissions need to be reduced is still needed. However, even if an education campaign occurs, there is still likely to remain a discrepancy between people's knowledge and understanding of climate change and their behaviour, i.e. actually living a sustainable lifestyle. This is where social marketing techniques will have to be use, as discussed in the previous chapter.

This lack of understanding creates a major obstacle in the way of VUW becoming carbon neutral. If the University Council and senior management fail to understand the urgency of climate change (as noted in Section 2.1), and the need for comprehensive action to address it, then a commitment to carbon neutrality is less likely. Ultimately, an education campaign on the importance of VUW becoming carbon neutral is needed for widespread buy-in.

\subsection{Benefits and opportunities of carbon neutrality at VUW}

The benefits and opportunities associated with VUW becoming carbon neutral identified by the interviewees are similar to benefits and opportunities identified in the literature regarding campus sustainability issues in Chapter 3. These were categorised into either the 'enlightened selfinterest' rationale or a short-term benefit rationale. Proponents of environmental initiatives often try to encourage organisations to adopt these environmental initiatives by emphasising the shortterm benefits, such as the financial savings; however, the literature suggests that successful longterm initiatives concentrate on the enlightened self-interest rationale. This in itself can pose a barrier, as it may prove more difficult to convince the University Council that the main reason why VUW should become carbon neutral is because it is the 'right thing' to do, rather than because of the short-term gains associated from it, such as financial savings or the marketing opportunities.

Objective 8 of the University's strategic plan states that the University will 'attract and retain high quality staff to support the achievement of the University's Vision and Strategic Objectives 
(VUW, 2006b). One of the main benefits identified by the literature on CSR initiatives is that organisations are able to fulfil such an objective. The enlightened self-interest rationale suggests that employees are more willing to work for and remain at organisations that incorporate CSR initiatives at their workplace. A carbon neutral initiative will not only fit the requirements of the VUW Environmental Policy, but also the requirements of the University's Vision and Strategic Objectives.

Strategic Objective 16 of VUW is to "strengthen its links with the Wellington community, national and international institutions'. By committing to carbon neutrality, VUW can help fulfill this objective, by working with the WCC on its own commitment to carbon neutrality, working with the New Zealand Government, and taking a leadership role in helping New Zealand reach a carbon neutral status. It can also strengthen its links with other international institutions who have already committed to carbon neutrality and be part of a global drive to lead society towards a low-carbon economy by 'walking the talk'.

The carbon neutral framework for VUW developed from the results of interviews parallels both the literature and empirical evidence from other organisations that have committed to carbon neutrality. Implementing a carbon neutral framework requires a process of continual improvement in economic, social, and environmental performance that should be made through incremental steps. It will require major efforts by key stakeholders of the university community. Funding and resources must be allocated for sustainability initiatives. This will confirm a firm commitment from the University Council and senior management, and therefore guarantee a more successful outcome.

\subsection{Barriers in the way of VUW becoming carbon neutral}

The majority of barriers identified by the interviewees can be classed as industrial barriers. This is a reflection of very nature of VUW as a whole. As explained in the literature review in Chapter 3, the industrial barriers reflect the special and unique features in which VUW engages, focusing on research and teaching. There is the tension between allocating resources to this main focus and to other objectives.

The organisational barriers identified by the interviewees are based on the individual nature of various stakeholders. For instance, the commitment needed to a carbon neutral initiative is likely 
to require a decision from a few members of the University Council. It cannot be classed as an industrial barrier, because universities in the USA have pledged to become carbon neutral. Therefore, the unique nature of a university cannot be a justification on why VUW cannot commit to a carbon neutral initiative.

Funding was identified by interviewees as the main obstacle in the way of successfully implementing a carbon neutral initiative at VUW. CarboNZero estimated a cost of $\$ 330000$ to offset the university's 2006 GHG emissions. This figure can be compared to the VUW surplus for 2006, before unusual items, which was \$4.0 million (\$1.4 million lower than budget and \$4.1 million less than the previous year). The reduction in the surplus reflected the growth of costs at a rate greater than growth in revenue. Clearly, VUW has to make careful choices in budget allocation, due to Government funding not being as much as the University (VUW, 2006b).

A key issue is that funding from the government does not match the overall expenses faced by VUW. In September 2006, the University Council decided to increase domestic undergraduate fees for 2007 by 5 percent and increase fees for postgraduate courses by $\$ 500$. This was deemed by VUW to be justified in continuing to be a high quality research-led university as required by the University Charter (VUW, 2006b) ${ }^{10}$.

However, the cost of being carbon neutral should not be seen as an extra liability that is extremely discretionary and can be ignored. VUW may have to internalise, due to the proposed NZ ETS, the costs of its GHG emissions to the extent that it buys energy directly and indirectly and this will affect its operations. As discussed in Chapter 2, cost increases may happen from next year, when the costs due to GHG emissions produced by liquid fossil fuels may be incorporated into the New Zealand economy, followed by emissions produced by stationary energy in 2010. Going carbon neutral anticipates these price signals and aims to cut emissions earlier. Even with the ETS's added costs, VUW may have to become carbon neutral in future, due to government regulation, as the government has signalled its intention that all public sector institutions will eventually become carbon neutral.

In line with the Tertiary Education Strategy and funding allocation, universities focus on research and teaching. VUW's own charter states that it is a research-led university. This creates a tension

\footnotetext{
${ }^{10}$ Data for 2007 was not available, as the 2007 VUW Annual Report had not been released at the time of writing.
} 
between research led outputs and universities as agents of change. However, the issues underlining funding are the reasons that intensify this tension. As higher education has become increasingly expensive, with most funding coming from public sources, society has taken a stronger interest in higher education. Traditional concepts of academic autonomy can be called into question. The results from the interviews illustrate that while it may seem appropriate to invest time and money in such areas as the Climate Change Research Institute, for VUW to show real leadership, it has to be seen to properly manage and reduce emissions produced from its operations.

Ultimately, convincing the University Council is a necessary step in VUW becoming carbon neutral. The University Council not only makes broad strategic decisions for VUW, but will play an important part in directing this initiative. Therefore it is vital to make clear the reasons why VUW has to address its GHG emissions through its operations. Not only will moving to carbon neutrality demonstrate the much-needed leadership for change, but will save the university money over time through its operations, thus offsetting the upfront cost of carbon neutralisation. Overcoming the perception barriers will determine whether VUW can become carbon neutral.

If VUW chose the carboNZero programme to become carbon neutral, it could be seen that investing \$330 000 into the programme is more expensive than purchasing offsets from other GHG offset markets. However, investing in a New Zealand company such as carboNZero will help fund further research and development in New Zealand. Offsets purchased through carboNZero will increase New Zealand carbon sinks, through EBEX 21, and will therefore reduce New Zealand's total GHG footprint.

\subsection{The carbon neutral framework}

The interviewees identified a framework that VUW could adopt if it chooses to commit to carbon neutrality. This indicates that there are two main options available for VUW to become carbon neutral. The first option is becoming carbon neutral by measuring VUW's carbon footprint each year, then purchasing offsets through the carboNZero programme discussed above. This would mean that VUW could become carbon neutral in a relativity short timeframe. The second option would be committing to carbon neutrality over a longer timeframe, reducing emissions where possible, implement behavioural change programmes, and then purchase offsets for emissions that cannot be reduced. 


\subsection{VUW as an agent of change}

The research and teaching produced by universities has played an important role in responding to climate change throughout the world. On a major strategic issue such as climate change, it could be expected that universities would be leading the way, in a transition to a low-carbon economy. However, as explained in Chapter 3, there is reluctance in VUW, as it appears in other New Zealand universities, to commit and demonstrate this leadership. Adopting a carbon neutral initiative is an ideal way in which VUW can take a leadership role in society. However, this leadership role is much more comprehensive than simply having the right courses or practices. In order to overcome the main industrial barriers mentioned in the interviewees, there needs to be a shift in the fundamental thinking regarding basic issues about the role of the university in society, creating a stronger relationship between climate friendly practices and the goals of VUW.

The basic structure of the university has remained the same for over 800 years. In order for a university to realistically be able to tackle the climate change crisis, it will require a redesign of operational institutional systems, the creation of an appropriate connection between operational and academic functions, and finding the appropriate mix of disciplinary and multidisciplinary programmes. This will require a major mind shift amongst not only academics and students, but also those who hold the most power at universities, the University Council and Vice Chancellor. This last step may pose the greatest barrier of all, and in all probability, may not be overcome anytime in the near future.

In the end, it will also require a combined effort with both central and local government. If the NZ Government's stance on carbon neutrality is to be achieved, then the role of New Zealand's universities, including VUW, will play a big part in this, through research and advice. Therefore, adequate funding is needed for New Zealand universities to not only become carbon neutral, but also be able to make climate change a top priority in teachings, research, and operations.

Local government, both the WCC and the Greater Wellington Regional Council, will have to work with VUW. In order to reduce the university's transport GHG footprint, investment has to be made into public transport. For Wellington City to achieve a carbon neutral status, it will have to work closely with the University, as the University is one of the regions largest employers, as well as a source of expertise on emission reductions. 
For that reason, for VUW to take on the role of a leader and agent of change, it will have to work closely with other organisations. Adopting a CSR initiative will involve more than the university's internal community stakeholders, but a variety of external ones too.

\subsection{Limitations of research}

The analysis and discussion of this thesis are based on the obtained as well as underpinning interviews and literature. It has to be acknowledged that findings are limited and may not represent a true and complete picture. Although the interviewees chosen for this thesis represent a wide range of VUW's community, this does not necessarily mean a fair representation. Further interviews were requested - including the Vice Chancellor. However, these were declined or there was no response to the request. More interviews will need to be conducted to achieve a more complete answer. Ideally, more members of the staff, the Senior Management Team and the University Council would have been interviewed.

Further investigations can also be carried out into whether there exist other universities, either domestically or internationally, which have already gone through the process of deciding whether or not to become carbon neutral, and then have chosen not to. What the reasons behind the decision and were alternative options chosen instead?

Students deciding which university to attend base their decisions on a number of factors; however there is limited research on whether environmental sustainability is included in this decisionmaking process. Surveying both current and prospective students on whether university environment sustainability plays a role in their decision on which university to attend could add to this research. 


\section{Conclusion}

The scientific evidence is clear-cut. Anthropogenic GHG emissions are changing the climate. Universities are at the forefront of research and teaching on climate change. However, there is reluctance amongst universities to integrate what is learnt from this teaching and research into their daily operations. Only recently have some international universities committed to carbon neutrality or emissions reduction measures.

Universities are the only organisations that are able to take a strong leadership role when questioning the status quo. They are able to take risks where other organisations, such as business and government, cannot. They have the expertise, freedom, and flexibility to make mistakes.

This research has found that VUW is in a position to commit to a carbon neutral initiative and therefore contribute zero net GHG emissions to the atmosphere. VUW has already set in place many of the requirements necessary to adoption of a carbon neutral initiative: by implementing an environmental policy and conducting yearly carbon audits of its operations. However, to actually achieve this objective will require a firm commitment from the University Council and senior management.

A carbon neutral initiative is a first step toward demonstrating that VUW is serious about climate change; VUW can show society a practical way to deal with GHG emissions. Furthermore, implementing such a policy offers a number of benefits and opportunities for VUW. However, in order to capitalise on these benefits and opportunities, VUW needs to overcome several barriers. As such, VUW will need to create a framework in which this initiative can be achieved.

The introduction of appropriate measures to deal with the GHG emissions produced from VUW's operations will be consistent with both the government' and WCC's intention to become carbon neutral.

Carbon neutrality can, however, be criticised if 'BAU' are maintained with offsets purchased to reach neutrality. The research has suggested that VUW can take a strong leadership role, but has to go beyond the "BAU" option. It will have to incorporate this initiative as broadly as possible into its operations. For this initiative to be successful, carbon neutrality will have to become a major strategy. 


\section{References}

ACUPCC. Dautremont-Smith, J., Cortese, A. Dyer, G., and Walton, J. (2007). Implementation Guide: Information and Resources for Participating Institutions.

http://www.presidentsclimatecommitment.org/pdf/ACUPCC_IG_Final.pdf: accessed $1^{\text {st }}$ November 2007.

Ahmed, F., Brown, J., Felix, D., Haurin, T., Seto B. (2005). Campus Climate Neutral. California: University of California, Santa Barbara

Alexander, R. Hope, M., and Degg M. (2007). Mainstreaming Sustainable Development - A Case Study: Ashton Hayes is going Carbon Neutral. Local Economy, 22, 91), 62-74.

Altbach, P. (1972). University Reform. Cambridge, Massachusetts. Schenkman Publishing Company.

Anderson, D. (2008). Energy Conservation at UCL. UCL News. http://www.ucl.ac.uk/news/newsarticles/0801/08010902: accessed $13^{\text {th }}$ January 2008.

Arrhenius, S. 1896. On the Influence of Carbonic Acid in the Air upon the Temperature of the Ground. Philosophical Magazine and Journal of Science, 5, (41), 237-275

Asquith, C. (2007). Going Green Equals Good Business. Diverse Issues in Higher Education, 24, (6), 14-15.

Aupperle, K., Carroll, A., Hatfield, J. (1985). An Empirical Examination of the Relationship between Corporate Social Responsibility and Profitability. The Academy of Management Journal, 28, (2), 446-463.

Balabanis, G., Philips, H., Lyall, J. (1998). Corporate Social Responsibility and Economic Performance in the Top British Companies: are they linked? European Business Review, 98, (1), 25-44.

Ball, A, Hughes, P. and Milne, M. (2007). Will Carbon-Neutral Public Services Help Stop Climate Change? Public Management and Policy Association review, 39, 6-8.

Bardati, D. (2006). The Integrative Role of the Campus Environmental Audit: Experiences at Bishop's University, Canada. International Journal of Sustainability in Higher Education, 7, (1), 57-68.

Barker, P. (2007). Co-leader of Gecko, the VUW Student Environmental Club. Personal Communication, Interview with Philip Barker $12^{\text {th }}$ July 2007.

Beaglehole, T. (2006). Speech at Launch of Confronting Climate Change, Nov 2006.

Begley, M. (2007). Executive Assistant \& Office Manager, Second Nature. Personal Communication, Email with Megan Begley: 17th October 2007.

Bekessy, S., Samson, K., and Clarkson, R. (2007). The Failure of Non-Binding Declarations to achieve University Sustainability: A Need for Accountability. International Journal of Sustainability in Higher Education, 8, (2), 301316.

Bentley, J. (2007). Director, Facilities Management. Personal Communication, Interview with Jenny Bentley 23rd July 2007.

Biddy, D. (2007). Pro-Vice Chancellor, Dean of Science, Dean of Architecture and Design. Personal Communication, Interview with Professor David Bibby $12^{\text {th }}$ September.

Bode, S. (2006). Long-Term Greenhouse Gas Emission Reductions- What's Possible, What's Necessary. Energy Policy, 34, (9), 971-993.

Boston, J. (2007). Post-2012: Towards a New Global Climate Treaty. Post-2012: Towards a New Global Climate Treaty. Wellington: Institute of Policy Studies 
Boston, J. (2007). Deputy Director of the Institute of Policy Studies. Personal Communication, Interview with Professor Jonathan Boston $10^{\text {th }}$ August 2007.

Breyman, S. (1999). Sustainability through Incremental Steps? The Case of Campus Greening at Rensselaer. Sustainability and University Life. W.L. Filho, (Ed). New York: Peter Lang Publishing, 79-87.

Callender, G. (1938). The Artificial Production of Carbon Dioxide and its Influence on Temperature. Quarterly Journal of the Royal Meteorological Society, 64, 223-240.

carboNZero. (2007a). Certified organisations.

http://www.carbonzero.co.nz/members/organisations_certified.asp\#Meridian ; accessed $19^{\text {th }}$ November 2007.

carboNZero. (2007b). Cost Estimated for carboNZero certification VUW. Environmental Committee Documentation, prepared by carboNZero, 20 September 2007.

Chapman, R., Boston, B., and Schwass, M. (2006). Confronting Climate Change: Critical Issues for New Zealand. Wellington: Victoria University Press.

Chapman, R. and Boston, J. (2007). The Nature of the Problem and the Case for Rapid Stabilisation. In Boston, J. (Ed.). Post-2012: Towards a New Global Climate Treaty. Wellington: Institute of Policy Studies

Chernushenko, D. (1996). Greening Campuses: Environmental Citizenship for Colleges and Universities. Winnipeg: International Institute for Sustainable Development.

Clark, H. (2007). Prime Minister's Statement to Parliament.

http://www.beehive.govt.nz/node/28357: accessed 23 ${ }^{\text {rd }}$ March 2007

Clarke, A. (2006). The Campus Environmental Management System Cycle in Practice: 15 years of Environmental Management, Education and Research at Dalhousie University. International Journal of Sustainability in Higher Education, 7, (4), 374 - 389.

Clean Air-Cool Planet. (2000). Campus for climate Action. http://www.cleanair-coolplanet.org/for_campuses.php: accessed 17th March 2007.

Clugston, R. and Calder, W. (1999). Critical Dimensions of Sustainability in Higher education. In Filho, W. (Eds). Sustainability and University Life. Frankfurt: Peter Lang

Cochran, P. (2007). The Evolution of Corporate Social Responsibility. Business Horizons, 50, (6), 449-454.

Comm, C. and Mathaisel, D. (2003). A Case Study of the Implications of Faculty Workload and Compensation for Improving Academic Quality. The International Journal of Education Management, 17, (5), 200-210.

Concept Consulting Group (CC Group). (2004). Electricity Emissions Factor Review - Prepared for NZ Climate Change Office. http://www.concept.co.nz/uploads/00039.pdf: accessed 10 ${ }^{\text {th }}$ August 2007.

Cooper, S., and Owen, D. (2007). Corporate Social Reporting and Stakeholder Accountability: The Missing Link. Accounting, Organizations and Society, 32, 649-667.

Conway, T., Dalton, C., Loo, J., and Benakoun, L. (2008). Developing Ecological Footprint Scenarios on University Campuses: A Case Study at the University of Toronto at Mississauga. International Journal of Sustainability in Higher Education, 9, (1), 4-20.

Cohen, S., Demeritt, D., Robinson, J., Rothman, D. (1998). Climate Change and Sustainable Development towards Dialogue. Global Environmental Change, 8, (4), 341-371.

Comm, C. and Mathaisel, D. (2003). An Exploratory Study of Best Lean Sustainability Practices in Higher Education. Quality Assurance in Education, 13, (3), 227-240.

Cortese, A. (2003). The Critical Role of Higher Education in Creating a Sustainable Future. Planning for a Higher Education, March-May, 15-22. 
Creighton, S. (1998). Greening the Ivory Tower - Improving the Environmental Track record of Universities, Colleges, and Other Institutions. Cambridge, Massachusetts: The MIT Press.

Creswell, J., and Plano Clark, V. (2007). Designing and Conducting Mixed Methods Research. Thousand Oaks, California: Sage Publications

Cruz, J. (2006). Dynamics of Supply Chain Networks with Corporate Social Responsibility through Environmental Decision-Making. European Journal of Operational Research, 184, (3), 1005-1031.

Dagan, M. (2002). A Summary of Energy Consumption and Greenhouse Gas Emissions at Middlebury College. Report. http://www.middlebury.edu/NR/rdonlyres/26273E73-9E1D-4283-864C1C6E9E5B74F9/0/Emissions_InvD_dagan.pdf: accessed 10 ${ }^{\text {th }}$ July 2007.

Dahle, M. and Neumayer, E. (2001). Overcoming Barriers to Campus Greening: A Survey among Higher Education Institutions in London, UK. International Journal of Sustainability in Higher Education, 2, (2), 139-160.

Daugherty, C. (2007). Assistant Vice-Chancellor (Research). Personal Communication, Interview with Professor Charles Daugherty $16^{\text {th }}$ October 2007.

Dautremont-Smith, J. (2003). Strategies for Institutional Kyoto Compliance: A Case Study of the Lewis and Clark Experience. International Journal of Sustainability in Higher Education, 4, (3), 257-262.

Dix, R. (2007). Manager, Student Union Complex. Personal Communication, Interview with Rainsforth Dix $7^{\text {th }}$ August 2007.

DJSI. (2008). Dow Jones Sustainability World Index, February 2008. http://www.sustainabilityindexes.com/djsi_pdf/publications/Factsheets/SAM_IndexesMonthly DJSIWorld.pdf: accessed $2^{\text {nd }}$ March 2008.

Dunn, K. (2005). Interviewing. In. I. Hay (Ed.). Qualitative Research Methods in Human Geography (Vol. 2). New York: Oxford Press.

Duggan, K. (2007). Manager, Information Analyst. Personal Communication, Interview with Kevin Duggan $17^{\text {th }}$ September 2007.

EPA. (1995). Introduction to AP 42, 1, $5^{\text {th }}$ Ed. http://www.epa.gov/ttn/chief/ap42/index.html: accessed $12^{\text {th }}$ May 2007.

Fawcett, T. (2005). Energy Use and Carbon Emissions from the Higher Education Sector. Report, Environmental Change Institute, University of Oxford. http://www.ukerc.ac.uk/Downloads/PDF/05/0511_HE_paper.pdf: accessed $13^{\text {th }}$ January 2008.

Fisher, J. (2003). Surface and Deep Approaches to Business Ethics. Leadership and Organisation Development Journal, 24, (2), 96-101.

Fontanier, G. (2007). 2007 International Officer, VUWSA. Personal Communication, Interview with Genevieve Fontanier, $5^{\text {th }}$ December 2007.

Golub, A., Markandya, A. and Marcellino, D. (2006). Does the Kyoto Protocol Cost too much and Create Unbreakable Barriers for Economic Growth? Contemporary Economic Policy, 24, (4), 520-535.

Gray, R. and Milne, M. (2004). Towards Reporting on the Triple Bottom Line: Mirages. Methods and Myths. In A. Henriques and A. Richardson (Eds). The triple Bottom Line: Does it all add up? London: Earthscan.

Grimble, R. and Wellard, K., Stakeholder Methodologies in Natural Resource Management. A Review of Principles, Contexts, Experiences and Opportunities. Agricultural Systems Journal, 55, (2), 173-193.

Hansen, J., Fung, I, Lacis, A., Rind, D., Lebedeff, S., Ruedy, R., Russell, G. and Stone, P. (1988). Global Climate Changes as Forecast by Goddard Institute for Space Studies Three-Dimensional Model. Journal of Geophysical Research, 93, (D3), 9341-9364. 
Hansen, J., Sato, M., Kharecha, P., Beerling, D., Masson-Delmotte, V., Pagani, M., Raymo, M., Royer, D., and Zachos, J. (2008). Target Atmospheric $\mathrm{CO}_{2}$ : Where Should Humanity Aim? Draft version.

http://www.columbia.edu/\%7Ejeh1/2008/TargetCO2_20080317.pdf: accessed 20th March 2008.

Hart, S. (1995). A Natural-Resource-Based View of the Firm. Academy of Management Review, 20, (4), 986-1014.

Henderson, D. (2001). The Case against ‘Corporate Social Responsibility’. Policy. 17, (2), 28-32.

Heede, R. and Swisher, J. (2002). Oberlin College: Climate Neutral by 2020. Report. Snowmass, Colorado: Rocky Mountain Institute.

Henriques, I. and Sadorsky, P. (1999). The Relationship between Environmental Commitment and Managerial Perceptions of Stakeholder Importance. The Academy of Management Journal, 42, (1), 87-99.

Hodgson, P. (2005). Carbon Tax Speech. http://www.beehive.govt.nz/?q=node/22886: accessed $3^{\text {rd }}$ May 2007.

Howden-Chapman, P. (2006). Inaugural Professorial Lecture: Thursday 17 August 2006.

http://www.otago.ac.nz/news/inaugural_lectures/2006/Howden-Chapman_3_book_final.pdf: accessed $3{ }^{\text {rd }}$ February 2007.

Hussain, S. (1999). The Ethics of ‘Going Green’: the Corporate Social Responsibility Debate. Business strategy and the environment, 8, (4), 203-210.

Hutton, M. (2007). Former-Climate Change Campaigner, WWF. Personal Communication, Interview with Melanie Hutton $10^{\text {th }}$ July 2007.

ICC. (1991). An International Chamber of Commerce Guide to Effective Environmental Auditing. Paris: ICC Publishing.

Ingenbleek, P., Binnekamp, M. and Goddijn, S. (2007). Setting Standards for CSR: A Comparative Case Study on Criteria-Formulating Organizations. Journal of Business Research, 60, (5), 539-548.

IPCC. (2005). Carbon Dioxide Capture and Storage.

http://www.ipcc.ch/pdf/special-reports/srccs/srccs_wholereport.pdf: accessed 12 June 2007.

IPCC. (2006). 2006 IPCC Guidelines for National Greenhouse Gas Inventories, Vol. 1.

http://www.ipcc-nggip.iges.or.jp/public/2006gl/pdf/1_Volume1/V1_1_Ch1_Introduction.pdf: accessed $13^{\text {th }}$ May 2007.

IPCC (2007a). Summary for Policymakers. Climate Change 2007: The Physical Science Basis. Contribution of Working Group I to the Fourth Assessment Report of the Intergovernmental Panel on Climate Change. http://ipcc-wg1.ucar.edu/wg1/Report/AR4WG1_Print_SPM.pdf: accessed $3^{\text {rd }}$ March 2007

IPCC. (2007b). Australia and New Zealand. Climate Change 2007: Impacts, Adaptation and Vulnerability. Contribution of Working Group II to the Fourth Assessment Report of the Intergovernmental Panel on Climate Change.

http://www.ipcc.ch/pdf/assessment-report/ar4/wg2/ar4-wg2-chapter11.pdf: accessed $1^{\text {st }}$ July 2007

IPCC. (2007c). Summary for Policymakers. Climate Change 2007: Synthesis Report.

http://www.ipcc.ch/pdf/assessment-report/ar4/syr/ar4_syr_spm.pdf; accessed $11^{\text {th }}$ August 2007

IPCC. (2007d). Issues Related to Mitigation in the Long Term Context. Climate Change 2007: Mitigation.

Contribution of Working Group III to the Fourth Assessment Report of the Inter-governmental.

http://www.ipcc.ch/pdf/assessment-report/ar4/wg3/ar4-wg3-chapter3.pdf: accessed 14 ${ }^{\text {th }}$ September 2007

Isham, J., Del Negro, L., Bission, C. (2003). Carbon Neutrality at Middlebury College: A compilation of Potential Objectives and Strategies to Minimize Campus Climate Impact. Vermont: Middlebury College Publishing

Jacob, D. (1999). Introduction to Atmospheric Chemistry. Princeton: Princeton University Press. 
Karlik-Neale, M. (2008). Sustainability Consultant, URS New Zealand. Personal Communication, Email with Marta Karlik-Neale: $11^{\text {th }}$ February 2008.

Klein, R., Schipper, E., Dessai, S. (2005). Integrating Mitigation and Adaptation into Climate and Development Policy: Three Research Questions. Environmental Science and Policy, 8, (6), 579-588.

Kloman, B. (2007). Middlebury College commits to becoming Carbon Neutral by 2016: Board of Trustees approves Student-Driven Proposal with Administration's Support.

http://www.middlebury.edu/about/pubaff/news_releases/2007/pubaff_633141333185905594.htm: accessed $12^{\text {th }}$ June 2007.

Levy, J. and Dilwali, K. (2000). Economic Incentives for Sustainable Resource Consumption at a Large University Past Performance and Future Considerations. International Journal of Sustainability in Higher Eduaction, 1, (3), 252-266.

Levin, J. (2006). UCSB Recognised as Climate Action Leader by California Registry. California Climate Action Registry: Press release. http://sustainability.ucsb.edu/_client/pdf/pr/UCSB_Climate_Action_Leader_051706.pdf: accessed $16^{\text {th }}$ May 2007.

Light, A., and Kats, E. (1996). Environmental Pragmatism. London and New York: Routledge.

Littlefield, C. (2007). Middlebury College, Vermont, USA. Personal Communication, Interview with Caitlin Littlefield 15th August 2007.

McKean, E. (2006). The New Oxford American Dictionary $\left(3^{\text {rd }}\right.$ Ed). New York: Oxford University Press.

McKenzie-Mohr, D. (2000). Promoting Sustainable Behavior: An Introduction to Community-Based Social Marketing. Journal of Social Issues, 56, (3), 543-554.

McKenzie-Mohr, D. and Smith, W. (1999). Fostering Sustainable Behaviour: and Introduction to Community-Based Social Marketing. Gabriole Island, B.C: New Society.

McQuillan, L. (2007). Waste audit at Rutherford House. Salient, 12 March, 2007. http://www.salient.org.nz/news/\%e2\%80\%98waste-audit\%e2\%80\%99-at-rutherford-house: accessed $19^{\text {th }}$ March 2007.

Malovics, G., Csigene, N. and Kraus, S. (2007). The Role of Corporate Social Responsibility in Strong Sustainability. The Journal of Socio-Economics, Article in Press, 1-12.

Marcell, K., Agyeman, J. and Rappaport, A. (2004). Cooling the Campus. Experiences from a Pilot Study to Reduce Electricity Use at Tufts University, USA, Using Social Marketing Methods. International Journal of Sustainability in Higher education, 5, (2), 169-189.

Martin, E. (2008). Manager, Special Projects, Harvard Green Campus Initiative. Personal Communication, Email with Emily Martin: $10^{\text {th }}$ February 2008.

Maltby, J. (1995). Environmental Audit: Theory and Practices. Managerial Auditing Journal, 10, (8), 15-26.

Manne, A., S. and Stephan, G., (2005). Global Climate Change and the Equity-Efficiency Puzzle. Energy, 30, (14), 2525-2536.

Marechai, K. (2007). The Economics of Climate Change and the Change of Climate in Economics. Energy Policy, $35,(10), 5181-5194$

MED. (2007). New Zealand Energy Greenhouse Gas Emissions 1990-2006. Wellington: Ministry of Economic Development. http://www.med.govt.nz/upload/49905/1_GHG_report.pdf: accessed 18 ${ }^{\text {th }}$ August 2007.

Montgomery, D., Bernstein, P., and Tuladhar, S. (2006). Potential for reducing Carbon Emissions from Non-Annex B Countries through Changes in Technology. Energy Economics, 28, (5-6), 742-762. 
Moir, L. (2001). What do We Mean by Corporate Social Responsibility? Corporate Governance, 1, (2), 16-22.

Monni, S., Syri, S. and Savolainen, I. (2004). Uncertainties in the Finnish Greenhouse Gas Emission Inventory. Environmental Science and Policy, 7, (2), 87-98.

Moskowitz, M. (1972). Choosing Socially-Responsible Stocks. Business and Society Review, 1, (1) 71-75

New Zealand Government. (2007). The Framework for a New Zealand Emissions Trading Scheme. Wellington: Ministry for the Environment and Treasury.

Nicolaides, A. (2006). The Implementation of Environmental Management towards Sustainable Universities and Education for Sustainable Development as an Ethical Imperative. International Journal of Sustainability in Higher Education, 7, (4), 414-424.

NZBCSD. (2002). The Challenge of Greenhouse Gas Emissions: The “why” and "how” of Accounting and Reporting for GHG Emissions- An industry guide.

http://www.nzbcsd.org.nz/climatechange/Climate_Change_Guide.pdf: accessed $3^{\text {rd }}$ May 2007.

Nyquist, S. (2000). Accounting Theory and Financial Environmental Reports. Eco-Management and Auditing, 7, (4), 178-185.

Oberlin. (2007). About Oberlin: Media Information.

http://www.oberlin.edu/newserv/facts.html: accessed $1^{\text {st }}$ July 2007.

Onisto, L. (1999). The Business of Sustainability. Ecological Economics 29, 37-43.

OPUS. (2007). Victoria University Travel Plan. Report, Auckland: Opus International Consultants Limited.

Orr, D. (1994). Earth in Mind: On Education, Environment and the Human Prospect. Washington: Island Press.

Orr, D. (2002). The Nature of Design: Ecology, Culture, and Human Intention. New York: Oxford University Press.

Parker, D. (2005). Carbon tax will not go ahead in 2007.

http://www.beehive.govt.nz/release/carbon+tax+will+not+go+ahead+2007: accessed $3^{\text {rd }}$ May 2007.

Parker, K. (1996). Pragmatism and Environmental Thought. In Light, A. and Katz, E. (Eds). Environmental Pragmatism. London and New York: Routledge.

Pearce, F. (2005). Histories: The Week the climate changed. New Scientist. 2521. http://environment.newscientist.com/channel/earth/mg18825210.600-histories-the-week-the-climate-changed.html: accessed $1^{\text {st }}$ March 2007.

Pearce, F. (2005). Look, No Footprints. New Scientist, 2594.

http://environment.newscientist.com/channel/earth/mg19325941.800-look-no-carbon-footprint.html: accessed $27^{\text {th }}$ March 2007.

Pierrehumbert, R. (2006). Climate change: A Catastrophe in Slow Motion. Chicago Journal of International Law, 6, (2), 573-596.

Pike, L, Shannon, T., Lawrimore, K., McGee, A., Taylor, M, and Lamoreaux, G. (2003). Science Education and Sustainability Initiatives. A Campus Recycling Case Study Shows the Importance of Opportunity. International Journal of Sustainability in Higher Education, 4, (3), 218-229.

Porter, M. and van der Linde, C. (1999). Green and Competitive: Ending the Stalemate. Journal of Business Administration and Policy Analysis.

http://info.cba.ksu.edu/sheu/MANGT810/MT810\%20SC\%20Reading/GreenCompetitive\%20Porter.pdf; accessed 17 ${ }^{\text {th }}$ December 2007.

Post, J. and Altman, B. (1994). Managing the Environmental Change Process: Barriers and Opportunities. Journal of Organizational Change Management. 7, (4), 64-81. 
Renowden, G. (2007). Hot Topic: Global warming and the future of New Zealand. Auckland, HB Media.

Revelle, R. and Suess, H., (1957). Carbon Dioxide Exchange between Atmosphere and Ocean and the Question of an Increase of Atmospheric $\mathrm{CO}_{2}$ during the Past Decades. Tellus, 9, (1), 18-27.

Rothrnberg, D. (1996). Laws of Nature vs. Laws of Respect. In Light, A. and Katz, E. (Eds). Environmental Pragmatism. London and New York: Routledge.

Rypdal, K. and Flugsrud, K. (2001). Sensitivity Analysis as a Tool for Systematic Reductions in Greenhouse Gas Inventory Uncertainties. Environmental Science and Policy, 4, (2), 117-135.

Samuelson, R., Wilkinson, D., Lawrence, S., Hammond, K., Walkington, M., and Pickup, M. (2007). Benefits-Cost Analysis of the New Zealand Energy Strategy. Ministry of Economic Development. http://www.med.govt.nz/upload/52236/Benefit-Cost\%20Analysis.pdf: accessed 12 $2^{\text {th }}$ January 2008.

Savage, G., Nix, T., Whitehead, C., and Blair, J. (1991). Startegies for Assessing and Managing Organisational Stakeholders. Academy of Management Executive, 5, (2), 61-75.

Shand, D. (2007). CCP-NZ. Personal Communication, Interview with Diana Shand 10th August 2007.

ShapeNZ. (2007). Public Favours Green Business (1 March 2007). NZBCSD, http://www.nzbcsd.org.nz/story.asp?id=740: accessed 15 ${ }^{\text {th }}$ July 2007.

Sharp, L. (2002). Green Campuses: the Road from Little Victories to Systemic Transformation. International Journal of Sustainability in Higher Education, 3, (2), 128-145.

Shearer, I. (2007). CCP-NZ. Personal Communication, Interview with Ian Shearer $10^{\text {th }}$ August 2007.

Shriberg, M. (2002). Sustainability in U.S. Higher Education: Organizational Factors Influencing Campus Environmental Performance and Leadership. A PhD Dissertation. The University of Michigan. http://sitemaker.umich.edu/snre-student-mshriber/files/shriberg.pdf: accessed $4^{\text {th }}$ March 2007.

Shrivastava, P. (1995). Industrial / Environmental Crises and Corporate Social Responsibility. The Journal of SocioEconomics, 24, (1), 211-227.

Shrivastava, P. and Hart, S. (1995), Creating Sustainable Corporations. Business strategy and the environment, 4, (3), 154-165.

Smith, A. (1993). Campus Ecology: A Guide to assessing Environmental Quality and creating Strategies for Change. Los Angeles. Living Planet Press.

Smith, K. (2007). The Carbon Neutral Myth: Offset Indulgences for your Climate Sins. Carbon Trade Watch. http://www.carbontradewatch.org/pubs/carbon_neutral_myth.pdf: accessed 14 ${ }^{\text {th }}$ March 2007.

Solomon, R. and Hanson, K. (1985). It's Good Business. New York: Harper Row.

Stanwick, P. and Stanwick, S. (1998). The Relationship between Corporate Social Performance and Organizational Size, Financial Performance, and Environmental Performance: An empirical examination. Journal of Business Ethics, 17, (2), 195-204.

Strachan, P. (1996). Achieving Environmental Excellence through Effective Teamwork. Team Performance Management: An International Journal, 2, (1), 25-29.

Stehr, N. and von Storch, H. (2005). Introduction to Papers on Mitigation and Adaptation Strategies for Climate Change: Protecting Nature from Society or protecting Society from Nature? Environmental Science and Policy, 8 , (6), 537-540. 
Stern, N. (2006). Stern Review Report on the Economics of Climate Change. Cambridge: Cambridge University Press. http://www.hmtreasury.gov.uk/independent_reviews/stern_review_economics_climate change/stern_review_report.cfm: accessed 1st March 2007

Sustainable Endowments Institute. (2007). College Sustainability Report Card: A Review of Campus and Endowment Polices at Leading Institutions.

http://www.endowmentinstitute.org/sustainability/CollegeSustainabilityReportCard2008.pdf: accessed $21^{\text {st }}$ September 2007.

Taiyab, N. (2006). Exploring the Market for Voluntary Carbon Offsets. International Institute for Environmental and Development (IIED).

http://www.iied.org/SM/eep/documents/MES8.pdf\#search=\%22exploring\%20the\%20market\%20for\%20developmen t\%20carbon\%22: accessed $23^{\text {rd }}$ September 2007.

TEC. (2006). The Second Tertiary Education Strategy 2007-12, Incorporating the Next Statement of Tertiary Education Priorities 2008-10. Wellington: Ministry of Education.

The Carbon Trust. (2006). Introducing Higher Education Carbon Management. United Kingdom: The Carbon Trust. http://www.carbontrust.co.uk/publications/publicationdetail?productid=PAC046: accessed $12^{\text {th }}$ September 2007.

The Carbon Trust. (2007). Further and Higher Education sector overview. United Kingdom: The Carbon Trust. http://www.carbontrust.co.uk/publications/publicationdetail?productid=CTV020: accessed 12 ${ }^{\text {th }}$ September 2007.

The Greenhouse Gas Protocol. (2004). A Corporate Accounting and Reporting Standard. Washington, USA and Conches-Geneva, Switzerland: World Resource Institute and World Business Council for Sustainable Development. http://pdf.wri.org/ghg_protocol_2004.pdf: accessed 10 ${ }^{\text {th }}$ March 2007.

Thomas, C., Tennant, T. and Rolls, J. (2000). The GHG Indicator: UNEP Guidelines for Calculating Greenhouse Gas Emissions for Businesses and Non-Commercial Organisations. New York: United Nations Environment Programme.

Thompson, R. and Green, W. (2005). When Sustainability is not a Priority: an analysis of Trends and Strategies. International Journal of Sustainability in Higher Education, 6, (1), 7-17.

Tyndall, J. (1861). On the Action of Gases and Vapours on Radiant Heat. TRILS, 1, 348-351.

Tietenberg, T. (2003). Environmental and Natural Resource Economics, $6^{\text {th }}$ Ed, New York: Addison Wesley.

Treasury. (2007). Price Estimation of Kyoto Compliant Emission Units. 30 June 2007. Wellington: New Zealand Government. http://www.treasury.govt.nz/government/liabilities/kyoto/carbonprice/kp-price-est-jun07.pdf: accessed $10^{\text {th }}$ January 2008.

UNCED. (1992). Agenda 21. http://www.un.org/esa/sustdev/documents/agenda21/index.htm: accessed 4th May 2007.

Uhl, C. and Anderson, A. (2001). Green Destiny: Universities leading the Way to a Sustainable Future, Bioscience, $51,(1), 36-42$.

Ullmann, A. (1985). Data in Search of a Theory: A Critical Examination of the Relationship among Social Performance, Social Disclosure, and Economic Performance, of U.S firms. Academy of Management Review, 10, (3), 540-557.

University of Cambridge. (2007). University of Cambridge 2006 Annual Report. http://www.admin.cam.ac.uk/univ/annualreport/2006/: accessed 14 ${ }^{\text {th }}$ November 2007.

UNFCCC. (1992). United Nations Framework Convention on Climate Change. http://unfccc.int/resource/docs/convkp/conveng.pdf: accessed $3^{\text {rd }}$ May 2007 
UNFCCC, (1998). Kyoto Protocol to the United Nations Framework Convention on Climate Change. http://unfccc.int/resource/docs/convkp/kpeng.pdf: accessed 3 ${ }^{\text {rd }}$ May 2007.

URS. (2007). Final Report Environmental Audit - prepared for Victoria University of Wellington. Wellington: URS New Zealand Limited

VCS. (2007). About the VCS. http://www.V-c-s.org/about.html: accessed $18^{\text {th }}$ December 2007.

Vine, E., Kats, G., Sathaye, J., and Joshi, H. (2003). International Greenhouse Gas Trading Programs: a Discussion of Measurement and Accounting Issues. Energy Policy, 31, (3), 211-224.

VUW. (2006a). Victoria University of Wellington Environmental Policy - Facilities Management Policy Group. http://policy.vuw.ac.nz/amphora! policy.vuw.ac.nz Policy 000000001296.pdf: accessed 15/03/07

VUW. (2006b). 2006 Annual Report. http://www.vuw.ac.nz/annualreports/: accessed 15 ${ }^{\text {th }}$ December 2007.

VUW. (2006). Victoria university of Wellington Campus Development Framework 2006- 2016.

http://www.victoria.ac.nz/home/about/newspubs/publications/campus_development_framework_final.pdf; accessed $2^{\text {nd }}$ September 2007.

VUW. (2007). University snapshot. http://www.victoria.ac.nz/home/about/snapshot.aspx: accessed $2^{\text {nd }}$ September 2007.

Ward, M., Hutton, M. and Renwick. (2007). Carbon Neutrality, Carbon Footprints, Offsets.....and Credibility. http://ips.ac.nz/events/downloads/C\%20Neutrality\%20Voluntary\%20Market\%20Offsets \%20and\%20Credibility.pdf: accessed $14^{\text {th }}$ Dec 2007.

Walley, N. and Whitehead, B. (1994). It's Not Easy Being Green. Harvard Business Review, 72, (3), 46-52.

Walker, D, Pitt, M, and Thakur, U. (2007). Environmental Management Systems: Information Management and Corporate Responsibility. Journal of Facilities Management, 5, (1), 49-61.

Walton, S. and Galea, C. (2005). Some Considerations for applying Business Sustainability Practices to Campus Environmental Challenges. International Journal of Sustainability in Higher Education, 6, (2), 147-160.

Wellington City Council. (2007a). Wellington City Proposes Carbon Neutral Vision. http://www.wellington.govt.nz/news/display-item.php?id=2933: accessed $2^{\text {nd }}$ June 2007.

Wellington City Council. (2007b). Council Aims for Carbon Neutrality by 2012. http://www.wellington.govt.nz/news/display-item.php?id=3083: accessed $7^{\text {th }}$ December 2007.

Williams, I. (2001). Environmental Chemistry a Modular Approach, Chichester: Wiley.

Wilks, A. (2007). Environmental Manager, Faculties Management, VUW. Personal Communication, Interview with Andrew Wilks $18^{\text {th }}$ July 2007.

Wilks, A. (2008). Environmental Manager, Faculties Management, VUW. Personal Communication, Email with Andrew Wilks (2006-2007 Emissions Summary), $18^{\text {th }}$ January 2008.

Winiwarter, W. and Rypdal, K. (2001). Assessing the Uncertainty Associated with National Greenhouse Gas Emission Inventories: a Case Study for Austria. Atmospheric Environment, 35, (32), 5425-5440.

World Business Council for Sustainable Development. (1999). Meeting Changing Expectations - Corporate Social Responsibility. http://www.wbcsd.org/DocRoot/hbdf19Txhmk3kDxBQDWW/CSRmeeting.pdf: accessed $21^{\text {st }}$ September 2007.

Wright, T. (2005). Giving "teeth" to an Environmental Policy: a Delphi Study at Dalhousie University. Journal of Cleaner Production, 14, (9-11), 761-768.

Yarr, R. (2007). Environment and Energy Manager, St. Andrews University. Personal Communication, Interview with Roddy Yarr, $27^{\text {th }}$ August 2007. 


\section{Appendix}

The following appendix contains:

1. List of Interviewees

2. Participant Information Sheet (VUW Stakeholder)

3. Participant Information Sheet (Other Organisations)

4. Informed Consent Form (VUW Stakeholders)

5. Informed Consent Form (Other Organisations)

6. Interview Questions (VUW Stakeholders0

7. Interview Questions (Other Organisations)

8. Ethic approval 


\section{Appendix 1: List of Interviews}

\section{VUW Stakeholders}

\begin{tabular}{|c|c|c|}
\hline Name & Position at VUW & Date \\
\hline Andrew Wilks & Environmental Manager & $18^{\text {th }}$ July 2007 \\
\hline Jenny Bentley & Director, Facilities Management & \\
\hline Rainsforth Dix & Manager, Student Union Complex & $7^{\text {th }}$ August 2007 \\
\hline Professor Charles Daugherty & Assistant Vice-Chancellor & $16^{\text {th }}$ October 2007 \\
\hline Professor David Bibby & Pro-Vice Chancellor, Dean of & \\
\hline Kevin Duggan & $\begin{array}{c}\text { Science, Dean of Architecture and } \\
\text { Design }\end{array}$ & $12^{\text {th }}$ September 2007 \\
\hline Professor Jonathan Boston & $\begin{array}{c}\text { Deputy Director of the Institute of } \\
\text { Policy Studies }\end{array}$ & $17^{\text {th }}$ September \\
\hline Philip Barker & Co-leader of Gecko, the VUW 2007 \\
\hline Genevieve Fontanier & student environmental club & $12^{\text {th }}$ July 2007 \\
\hline
\end{tabular}

\section{Representatives of other Organisations}

\begin{tabular}{|c|c|c|}
\hline Name & Position at VUW & Date \\
\hline Melanie Hutton & $\begin{array}{c}\text { Former-Climate Change } \\
\text { Campaigner, WWF }\end{array}$ & $10^{\text {th }}$ July 2007 \\
\hline Diana Shand & CCP-NZ & $10^{\text {th }}$ August 2007 \\
\hline Ian Shearer & CCP-NZ & $10^{\text {th }}$ August 2007 \\
\hline Caitlin Littlefield & Middlebury College, Vermont, USA. & $15^{\text {th }}$ August 2007 \\
\hline Roddy Yarr & St Andrews University, Scotland. & $27^{\text {th }}$ August 07 \\
\hline
\end{tabular}


Appendix 2: Participant Information Sheet (VUW Stakeholder)

Participant Information Sheet for a Study on "An assessment of whether a carbon neutral initiative can successfully be implemented at Victoria University of Wellington"

Researcher: Tushara Kodikara: School of Geography, Environment and Earth Science, Victoria University of Wellington.

I am a Masters student in Environmental Studies at Victoria University of Wellington (VUW). As part of this degree I am undertaking a research project leading to a thesis. The project is an assessment of whether a carbon neutral initiative can successfully be implemented at Victoria University of Wellington The University requires that ethics approval be obtained for research involving human participants.

The questions that I will ask will focus on climate change, and the benefits and barriers involved for VUW becoming carbon neutral.

I am inviting key stakeholders involved with decision-making at VUW to take part in this study. Participants will be asked a series of standardised open-ended questions. The interview will take no more than half an hour.

Participation is voluntary and all research findings will be reported with the intent of not being anonymous or confidential; however, participants will be given an option of being anonymous. Information collected will be kept secure. The results collected will be reported in my thesis, and a subsequently conference and paper. The thesis will be submitted for marking to the School of Geography, Environment and Earth Science, Victoria University of Wellington.

Participants have the opportunity to check interview notes and will be able to provide feedback. Feedback can be given any time, prior to final analysis of data. Should the participant feel the need to withdraw from the project, they may do so at any time before the data is analysed. Please contact me by email or phone call to do so.

If you have any questions or would like to receive further information about the project, please contact me at kodikatush@student.vuw.ac.nz, or by phone on 027356 4973, or through my supervisor, Associate Professor Ralph Chapman, at the School of Geography, Environment and Earth Science, Victoria University of Wellington.

Tushara Kodikara Signed: 


\section{Appendix 3: Participant Information Sheet (Other Organisations)}

Participant Information Sheet for a Study on "An assessment of whether a carbon neutral initiative can successfully be implemented at Victoria University of Wellington"

Researcher: Tushara Kodikara: School of Geography, Environment and Earth Science, Victoria University of Wellington.

I am a Masters student in Environmental Studies at Victoria University of Wellington (VUW). As part of this degree I am undertaking a research project leading to a thesis. The project is an assessment of whether a carbon neutral initiative can successfully be implemented at Victoria University of Wellington The University requires that ethics approval be obtained for research involving human participants.

The questions that I will ask will focus on climate change, and the benefits and barriers involved for VUW becoming carbon neutral.

I am inviting organisations that have already set a target of becoming carbon neutral take part in this study. Participants will be asked a series of standardised open ended questions. The interview will take no more than half an hour.

Participation is voluntary and all research findings will be reported with the intent of not being anonymous or confidential; however, participants will be given an option of being anonymous. Information collected will be kept secure. The results collected will be reported in my thesis, and a subsequently conference and paper. The thesis will be submitted for marking to the School of Geography, Environment and Earth Science, Victoria University of Wellington.

Participants have the opportunity to check interview notes, and will be able to provide feedback. Feedback can be given any time, prior to final analysis of data. Should the participant feel the need to withdraw from the project, they may do so at any time before the data is analysed. Please contact me by email or phone call to do so.

If you have any questions or would like to receive further information about the project, please contact me at kodikatush@student.vuw.ac.nz, or by phone on 027356 4973, or through my supervisor, Associate Professor Ralph Chapman, at the School of Geography, Environment and Earth Science, Victoria University of Wellington.

Tushara Kodikara Signed: 


\section{Appendix 4: Informed Consent Form (VUW Stakeholders)}

Title: An assessment of how Victoria University of Wellington can cost-effectively become carbon neutral.

\section{INFORMED CONSENT FORM}

Researcher: Tushara Kodikara

0273564973

Supervisor: Associate Professor Ralph Chapman

Director, Environmental Studies

Introduction

Human Ethics Committee, which has approved this research project, requires that all research involve Participants who are: 1) fully informed about the nature of the research; and 2) consent to participate. This "Informed Consent Form" has been designed in accordance with these requirements, to inform all Participants about the nature of the project and their participation in it. It is meant to ensure that research Participants and their communities are protected from any harm potentially arising from their participation in the research process.

Purpose of the Study

This study is part of a research project for a Masters Thesis and intends to answer the following question:

Whether a carbon neutral initiative can successfully be implemented at Victoria University of Wellington

(See also attached "Information Sheet")

Research Format

Qualitative field research will be undertaken at Victoria University of Wellington. No physical discomfort will be experienced by Participants. Questions will not be of a personal nature.

Interviews - Participants will be asked to take part in a standardised open-ended interview. Each interview will last up to approximately half an hour. 


\section{Appendix 5: Informed Consent Form (other organisations)}

Title: An assessment of how Victoria University of Wellington can cost effectively become carbon neutral.

\section{INFORMED CONSENT FORM}

Researcher: Tushara Kodikara 0273564973

Supervisor: Associate Professor Ralph Chapman Director, Environmental Studies

Introduction

Human Ethics Committee which has approved this research project requires that all research involve Participants who are: 1) fully informed about the nature of the research; and 2) consent to participate. This "Informed Consent Form" has been designed in accordance with these requirements, to inform all Participants about the nature of the project and their participation in it. It is meant to ensure that research Participants and their communities are protected from any harm potentially arising from their participation in the research process.

Purpose of the Study

This study is part of a research project for a Masters Thesis, and intends to answer the following question:

Whether a carbon neutral initiative can successfully be implemented at Victoria University of Wellington

(See also attached "Participant Information Sheet")

Research Format

Qualitative field research will be undertaken at various organisations that have set a target of becoming carbon neutral. No discomfort will be experienced by Participants. Questions would not be of a personal nature.

Interviews -Participants will be asked to take part in a standardised open-ended interview. Each interview will last up to approximately half an hour. 
Please circle one

I consent to information or opinions that I have given being attributed to me in any reports on this research.

$\mathrm{Y} / \mathrm{N}$

I understand that I will have an opportunity to check the transcript of the interview before publication.

$\mathrm{Y} / \mathrm{N}$

I understand that the data I provide will not be used for any other purpose or released to others without written consent.

$\mathrm{Y} / \mathrm{N}$

I would like to receive a summary of the results of this research when it is completed.

$\mathrm{Y} / \mathrm{N}$

I agree to take part in this research

Signatures Date:

Participant:

Name:

Signature:

Researcher:

I certify that this form and its attached "Participant Information Sheet" cover letter provide a complete and accurate description of the aims and processes of this research project.

Name:

Signature:

Date: 


\section{Appendix 6: Interview Questions (VUW)}

\section{VUW and Carbon Neutrality: Interview questions for VUW stakeholders}

Do you think there is a problem of climate change?

Do you consider it an urgent and important issue?

How would you rate your level of knowledge of climate change from 1 to 10 where 1 means 'knowing nothing at all' and 10 means 'a great deal?

Do you believe that VUW has demonstrated a willingness to address climate change in its teaching and research?

Do you believe that VUW teachers are showing enough knowledge of climate change in their courses?

What is your understanding of carbon neutrality for an organisation such as a university?

Do you believe that it is an achievable goal for VUW?

If yes, then what should the timeframe be? Should it follow other state sector organisations and have a target of becoming carbon neutral by 2012 ?

What should be done to get there?

Who is responsible?

What benefits do you see in carbon neutrality for VUW?

Who precisely benefits?

What are the costs involved for VUW?

What do you see as the barriers to achieving carbon neutrality for VUW?

How might these barriers be overcome?

Who should pay for VUW becoming carbon neutral?

How do you prioritise achieving carbon neutrality in relation to other objectives that VUW faces? What are these other objectives?

NOTE: As the interviews were conducted in a semi-structured style, not all the above questions were asked of each interviewee. 


\section{Appendix 7: Interview Questions (Other Organisations)}

Interview questions for representative of organisations that have set a target of carbon neutrality

Do you think there is a problem of climate change?

Do you consider it an urgent and important issue?

How would you rate your level of knowledge of climate change from 1 to 10 , where 1 means 'knowing nothing at all' and 10 means 'a great deal'?

How will your organisation be positioned in 10 years time, in regard to dealing with climate change?

What is your organisation doing to get to that position?

Who is responsible for this?

What is your understanding of carbon neutrality?

Why did your organisation set a target of becoming carbon neutral?

What steps are involved in becoming carbon neutral for your organisation?

What benefits do you see in carbon neutrality for your organisation?

Who benefits from your organisation becoming carbon neutral?

What were / are the costs involved for your organisation?

What were the barriers involved with your organisation adopting a carbon neutral target?

What are the barriers involved with your organisation implementing a carbon neutral target?

How are these implementation barriers overcome?

What is your mix of carbon emission reductions and offsetting measures?

How do you prioritise achieving carbon neutrality in respect of other objectives that your organisation faces? What are these other objectives?

NOTE: As the interviews were conducted in a semi-structured style, not all the above questions were asked of each interviewee. 